\title{
HALL ALGEBRAS OF CURVES, COMMUTING VARIETIES AND LANGLANDS DUALITY
}

\author{
O. SCHIFFMANN, E. VASSEROT
}

\begin{abstract}
We construct an isomorphism between the (universal) spherical Hall algebra of a smooth projective curve of genus $g$ and a convolution algebra in the (equivariant) $K$-theory of the genus $g$ commuting varieties $C_{\mathfrak{g l}_{r}}=\left\{\left(x_{i}, y_{i}\right) \in \mathfrak{g l}_{r}^{2 g} ; \sum_{i=1}^{g}\left[x_{i}, y_{i}\right]=0\right\}$. We can view this isomorphism as a version of the geometric Langlands duality in the formal neighborhood of the trivial local system, for the group $G L_{r}$. We extend this to all reductive groups and we compute the image, under our correspondence, of the skyscraper sheaf supported on the trivial local system.
\end{abstract}

\section{Contents}

$0 . \quad$ Introduction

1. Hall algebras of curves 5

2. K-theoretic Hall algebras 18

3 . The isomorphism 24

Appendix A. The principal Hall algebra 29

Appendix B. Generalization to arbitrary reductive groups 33

References

\section{INTRODUCTION}

0.1. Let $X$ be a smooth connected projective curve of genus $g$ defined over a finite field $\mathbb{F}_{q}$. Let $\mathrm{Bun}_{r} X$ stand for the set of all (isomorphism classes of) vector bundles of rank $r$ over $X$. Consider the vector space

$$
\mathbf{H}_{V e c(X)}=\bigoplus_{r \geq 1} \operatorname{Fun}\left(\operatorname{Bun}_{r} X, \mathbb{C}\right),
$$

where $F u n(\cdot, \mathbb{C})$ denotes the set of complex valued functions with finite support. Let $P \subset G L_{r}$ be a parabolic subgroup with Levi factor $L \simeq G L_{s} \times G L_{t}$. The convolution diagram

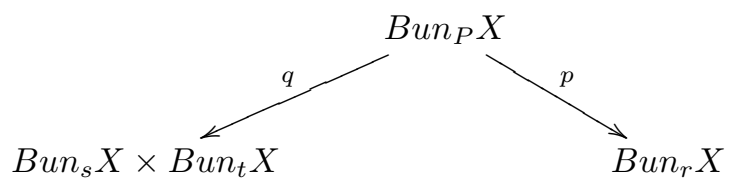

equips $\mathbf{H}_{V e c(X)}$ with an associative product and a coassociative coproduct. Now let $\operatorname{Coh}_{0} X$ stand for the set of all torsion coherent sheaves on $X$. A convolution diagram similar to (0.1) allows one to equip $H_{\operatorname{Tor}(X)}=F u n\left(\operatorname{Coh}_{0} X, \mathbb{C}\right)$ with the structure of an algebra (actually a Hopf algebra). This algebra acts on $\mathbf{H}_{V e c(X)}$. This construction has a natural interpretation in the theory of automorphic forms over function fields : $\mathbf{H}_{V e c(X)}$ is the direct sum (over $r$ ) of the spaces of unramified automorphic forms for the groups $G L_{r}$ over the ring of adèles of $X$; the product and coproduct in $\mathbf{H}_{V e c(X)}$ correspond to parabolic induction and constant term maps respectively; finally, $H_{\operatorname{Tor}(X)}$ is the algebra of (unramified) Hecke operators acting on the above automorphic forms. 
In $[\mathrm{K}]$, Kapranov initiated the systematic study of $\mathbf{H}_{V e c}(X)$, using the language of Hall algebras. The algebra $\mathbf{H}_{V e c(X)}$ is generated as by all cuspidal functions; Kapranov translated the functional equations satisfied by Eisenstein series associated to such cuspidal functions (or pairs of cuspidal functions) into commutation relations between the corresponding generators. These commutation relations bear a strong ressemblance with those appearing in Drinfeld's new realization of quantum affine algebras. In fact, Kapranov fully determined $\mathbf{H}_{V e c(X)}$ when $X=\mathbb{P}^{1}$ and identified it with the quantum group $\mathbf{U}_{v}^{+}\left(\widehat{\mathfrak{s l}}_{2}\right)$, where $v=q^{-1 / 2}$. The algebra $\mathbf{H}_{V e c(X)}$ is also explicitly described when $X$ is an elliptic curve, see [BS], [F1]. In this case it is related to double affine Hecke algebras, see [SV1].

0.2. In this paper, we let $X$ be a curve of arbitrary genus, but we restrict our attention to a subalgebra of $\mathbf{H}_{V e c(X)}$ generated by cuspidal functions of rank one. More precisely, we define the spherical Hall algebra $\mathbf{U}_{X}^{>}$to be the subalgebra of $\mathbf{H}_{V e c(X)}$ generated by the characteristic functions $1_{P i c^{d} X}$ for $d \in \mathbb{Z}$. Our motivation here is to understand the structure of this algebra, as well as its place in the Langlands program.

Our first result gives a combinatorial realization of $\mathbf{U}_{X}^{>}$in terms of shuffle algebras. Let $\alpha_{1}, \overline{\alpha_{1}}, \ldots, \alpha_{g}, \overline{\alpha_{g}}$ be the Weil numbers of $X$ and set

$$
g_{X}(z)=z^{g-1} \frac{1-q z}{1-z^{-1}} \prod_{i=1}^{g}\left(1-\alpha_{i} z^{-1}\right)\left(1-\bar{\alpha}_{i} z^{-1}\right)
$$

Let $\mathbb{C}\left(x_{1}, \ldots, x_{r}\right)^{\mathfrak{S}_{r}}$ stand for the space of symmetric rational functions in $r$ variables. We equip the graded space $\mathbf{V}=\mathbb{C} 1 \oplus \bigoplus_{r \geq 1} \mathbb{C}\left(x_{1}, \ldots, x_{r}\right)^{\mathfrak{S}_{r}}$ with the shuffle product

$$
P\left(x_{1}, \ldots, x_{r}\right) \star Q\left(x_{1}, \ldots, x_{s}\right)=\sum_{w \in S h_{r, s}} w\left(\prod_{\substack{1 \leqslant i \leqslant r \\ r+1 \leqslant j \leqslant r+s}} g_{X}\left(x_{i} / x_{j}\right) P\left(x_{1}, \ldots, x_{r}\right) Q\left(x_{r+1}, \ldots, x_{r+s}\right)\right)
$$

where $S h_{r, s} \subset \mathfrak{S}_{r+s}$ stands for the set of all $(r, s)$ shuffles on $r+s$ letters. Let $\mathbf{A}_{g_{X}(z)}$ be the subalgebra of $\mathbf{V}$ generated by $\mathbb{C}\left[x_{1}^{ \pm 1}\right] \subset \mathbf{V}_{1}$. It is a graded subalgebra $\mathbf{A}_{g_{X}(z)}=\bigoplus_{r \geq 0} \mathbf{A}_{r}$. Shuffle algebras of the above kind associated to rational functions have been studied in particular by B. Feigin and his collaborators, see e.g. [FO].

Theorem 1. The assignement $1_{P i c^{l} X} \mapsto x_{1}^{l}$ extends to an algebra isomorphism

$$
\Upsilon_{X}: \mathbf{U}_{X}^{>} \stackrel{\sim}{\longrightarrow} \mathbf{A}_{g_{X}(z)}
$$

The map $\Upsilon_{X}$ is essentially the constant term map (restriction to the torus), and Theorem 1 is a consequence of the Gindikin-Karpelevich formula. The fact that the constant term map lands in a space of symmetric functions is a manifestation of the functional equation for Eisenstein series. The Hecke operators preserve the spherical Hall algebra $\mathbf{U}_{X}^{>}$, and their action on each graded component $\mathbf{U}_{X}^{>}[r]$ is directly related to the action of $\mathbb{C}\left[x_{1}^{ \pm 1}, \ldots, x_{r}^{ \pm 1}\right]^{\mathfrak{S}_{r}} \simeq \operatorname{Rep} G L_{r}$ on $\mathbf{A}_{r}$. It would therefore be interesting to determine precisely the structure of $\mathbf{A}_{r}$ as a $\mathbb{C}\left[x_{1}^{ \pm 1}, \ldots, x_{r}^{ \pm 1}\right]^{\mathfrak{S}_{r}}$ module; a first step is taken in Proposition 1.14 where the support of $\mathbf{A}_{r}$ is determined in terms of wheel conditions.

A consequence of Theorem 1 is that $\mathbf{U}_{X}^{>}$only depends on the genus $g$ of $X$ and on its set of Weil numbers; this allows us to define, for each genus $g$, a 'universal' form $\mathbf{U}_{R_{a}}^{>}$of $\mathbf{U}_{X}^{>}$over the representation ring $R_{a}=\operatorname{Rep} T_{a}$ of the torus

$$
T_{a}=\left\{\left(\eta_{1}, \bar{\eta}_{1}, \ldots, \eta_{g}, \bar{\eta}_{g}\right) \in\left(\mathbb{C}^{\times}\right)^{2 g} ; \eta_{i} \bar{\eta}_{i}=\eta_{j} \bar{\eta}_{j}, \forall i, j\right\} \simeq\left(\mathbb{C}^{\times}\right)^{g+1} .
$$


0.3. The Langlands correspondence for function fields, proved for $G L_{r}$ by Drinfeld $(r=2)$ and then by L. Lafforgue (for all $r$ ) sets up a bijection between cuspidal Hecke eigenforms of rank $r$ over $X$ and irreducible $n$-dimensional $l$-adic representations of the Galois group $\operatorname{Gal}(\bar{F} / F)$ where $F$ is the function field of $X$. The only cuspidal Hecke eigenform belonging to the spherical Hall algebra $\mathbf{U}_{X}^{>}$is the constant function $1_{P i c X}$, which corresponds to the trivial (one-dimensional) Galois representation. Hence it is natural to expect that the Langlands correspondence relevant to $\mathbf{U}_{X}^{>}$will involve Galois representations 'close' to the trivial one.

At the moment we are not able to understand the role of the algebra $\mathbf{U}_{X}^{>}$in this Langlands picture for any fixed curve $X$. However, we will give such an interpretation for the universal spherical Hall algebra $\mathbf{U}_{R_{a}}^{>}$of a fixed genus $g$. For this, one has to pass to the complex setting and consider instead Beilinson and Drinfeld's version of the geometric Langlands program. Recall that this program aims at setting up an equivalence of (unbounded, triangulated) categories

$$
\operatorname{Coh}\left(\underline{\operatorname{Loc}}_{r} X_{\mathbb{C}}\right) \simeq D-\bmod \left(\underline{\operatorname{Bun}}_{r} X_{\mathbb{C}}\right)
$$

where $\underline{\operatorname{Loc}}_{r} X_{\mathbb{C}}$ and $\underline{B u n}_{r} X_{\mathbb{C}}$ are the stacks of rank $r$ local systems and rank $r$ vector bundles on a complex curve $X_{\mathbb{C}}$. When $r=1$ such an equivalence is known thanks to the work of Lang, Laumon and Rosenlicht (the geometric class field theory, see e.g. [L4]).

Let us consider the formal neighborhood $\widehat{\text { triv }}_{r}$ of the trivial local system triv $v_{r}$ in $\underline{\operatorname{Loc}}_{r} X_{\mathbb{C}}$ and let us assume for simplicity that $g>0$. By deformation theory $\widehat{\text { triv }}_{r}$ is isomorphic to the formal neighborhood of $\{0\}$ in the (singular) quotient stack $C_{\mathfrak{g l}_{r}} / G L_{r}$, where $C_{\mathfrak{g l}_{r}}$ is the quadratic cone of solutions to the Maurer-Cartan equation

$$
C_{\mathfrak{g l}_{r}}=\left\{u \in H^{1}\left(X_{\mathbb{C}}, \mathfrak{g l}_{r}\right) ;[u, u]=0\right\} .
$$

Here [, ] is the Lie bracket on the dg Lie algebra $H^{\bullet+1}\left(X_{\mathbb{C}}, \mathfrak{g l}_{r}\right)$ given by $\left[c \otimes x, c^{\prime} \otimes y\right]=c \cdot c^{\prime} \otimes[x, y]$. Fixing a symplectic basis of $H^{1}\left(X_{\mathbb{C}}, \mathbb{C}\right)$ yields an identification

$$
C_{\mathfrak{g l}_{r}}=\left\{\left(a_{i}, b_{i}\right)_{i=1, \ldots, g} \in\left(\mathfrak{g l}_{r}\right)^{2 g} ; \sum_{i}\left[a_{i}, b_{i}\right]=0\right\} .
$$

The symplectic group $S p\left(H^{1}\left(X_{\mathbb{C}}, \mathbb{C}\right), \cdot\right)$ naturally acts on $C_{\mathfrak{g l}_{r}} / G L_{r}$, and so does the torus

$$
T_{s}=\left\{\left(e_{1}, f_{1}, \ldots, e_{g}, f_{g}\right) \in\left(\mathbb{C}^{\times}\right)^{2 g} ; e_{i} f_{i}=e_{j} f_{j}, \forall i, j\right\} \simeq\left(\mathbb{C}^{\times}\right)^{g+1} .
$$

Let $P$ be a parabolic subgroup of $G L_{r}$ with Levi factor $G L_{s} \times G L_{t}$. Using the diagram

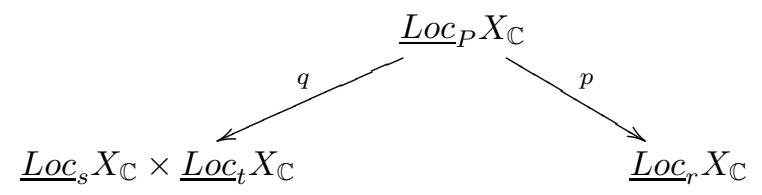

(and its restriction to formal neighborhoods of trivial local systems) we define a convolution product in equivariant $K$-theory

$$
K^{T_{s}}\left(C_{\mathfrak{g l}_{s}} / G L_{s}\right) \otimes K^{T_{s}}\left(C_{\mathfrak{g l}_{t}} / G L_{t}\right) \rightarrow K^{T_{s}}\left(C_{\mathfrak{g l}_{r}} / G L_{r}\right)
$$

This equips the graded space

$$
\mathbf{C}=\mathbb{C} 1 \oplus \bigoplus_{r \geq 1} K^{T_{s}}\left(C_{\mathfrak{g l}_{r}} / G L_{r}\right)
$$

with the structure of an associative algebra over the representation ring $R_{s}=\operatorname{Rep} T_{s}$. Observe that the formal neighborhood $\widehat{\text { riv }}_{r}$, and hence the algebra $\mathbf{C}$, only depend, up to isomorphism, on the genus $g$ of $X_{\mathbb{C}}$.

Let $\mathbf{C}^{\prime}$ be the subalgebra of $\mathbf{C}$ generated by $\mathbf{C}_{1}=K^{T_{s} \times G L_{1}}\left(\mathbb{C}^{2 g}\right)=R_{s}\left[x^{ \pm 1}\right]$. Inspired by the note $[\mathrm{Gr}]$, in the context of quivers, we prove the following result. Put

$$
k(z)=\frac{1-p^{-1} z^{-1}}{1-z} \prod_{i=1}^{g}\left(1-e_{i}^{-1} z\right)\left(1-f_{i}^{-1} z\right) \in R_{s}(z)
$$


where we have set $p=e_{i} f_{i}$ (for any $i$ ). Let $\mathbf{A}_{k(z)}$ be the $R_{s}$-shuffle algebra associated to $k(z)$.

Theorem 2. The assignment $x^{l} \mapsto x_{1}^{l}$ in degree one extends to an algebra isomorphism

$$
\Phi: \mathbf{C}^{\prime} / \text { torsion } \stackrel{\sim}{\longrightarrow} \mathbf{A}_{k(z)} .
$$

We conjecture that $\mathbf{C}^{\prime}$ is a torsion free module.

0.4. The functions $g(z)$ and $k(z)$ are of the same form. Combining Theorems 1 and 2 we deduce the following Langlands type isomorphism. We identify $R_{a}$ and $R_{s}$ via $\eta_{i} \mapsto e_{i}^{-1}, \bar{\eta}_{i} \mapsto f_{i}^{-1}$.

Corollary. There exists a unique algebra (anti)isomorphism

$$
\Theta_{R}: \mathbf{C}^{\prime} / \text { torsion } \stackrel{\sim}{\longrightarrow} \dot{\mathbf{U}}_{R_{a}}^{>}
$$

which restricts to geometric class field theory in degree one.

In other words, the (universal) spherical Hall algebra is in correspondence with a certain convolution algebra of coherent sheaves on the stacks $\underline{L o c}{ }_{r} X_{\mathbb{C}}$, supported in the formal neighborhood of the trivial local systems. The above corollary also suggests that the Langlands correspondence should be compatible with the parabolic induction functors constructed from diagrams (0.1) and (0.6) respectively. Note that we need to use a slightly twisted version $\dot{\mathbf{U}}_{R_{a}}^{>}$of $\mathbf{U}_{R_{a}}^{>}$, see Section 1.13. This twist is indeed very classical in the theory of Eisenstein series. Finally, one may show that the isomorphism $\Theta_{R}$ intertwines the actions of the Wilson and Hecke operators, as required of (geometric) Langlands duality.

Several remarks are in order at this point. First of all, the $K$-theoretic Hall algebra $\mathbf{C}^{\prime}$ is constructed out of the moduli stacks $\underline{\operatorname{Loc}}{ }_{r} X_{\mathbb{C}}$ for a complex curve $X_{\mathbb{C}}$ of genus $g$ while the spherical Hall algebra $\mathbf{U}_{R_{a}}^{>}$is defined using curves of genus $g$ over finite fields. Moreover, $\mathbf{C}^{\prime}$ is a Grothendieck group of $T_{s^{-}}$equivariant coherent sheaves while the structure of $\mathbf{U}_{R_{a}}^{>}$as an $R_{a^{-}}$ module comes from the Weil numbers of curves $X$ over $\mathbb{F}_{q}$. In order to correct this discrepancy, and to get something closer in spirit to (0.3) one is tempted to consider instead of $\mathbf{U}_{R_{a}}^{>}$the monoidal category $\mathcal{E} i s_{X_{\mathbb{C}}}$ of Eisenstein automorphic (perverse) sheaves over the stacks $\underline{B u n}_{r} X_{\mathbb{C}}$, see [L3], [S1]. We expect the symplectic group $S p\left(H^{1}\left(X_{\mathbb{C}}, \mathbb{C}\right), \cdot\right)$ and the torus $T_{a}$ to act on $\mathcal{E} i s_{X_{\mathbb{C}}}$, and we expect the resulting $T_{a}$-graded Grothendieck group to be isomorphic to (a completion of) $\mathbf{U}_{R_{a}}^{>}$. When $\mathbb{C}$ is replaced by $\overline{\mathbb{F}_{q}}$ the $T_{a}$-grading on $\mathcal{E} i s_{X_{\mathbb{F}_{q}}}$ should come from the Frobenius weight structure of the Eisenstein sheaves, see [S1] and [S3] for $g=1$. We hope to get back to this in the future.

We give two immediate applications of our Langlands isomorphism (0.7). We determine the image, under $\Theta_{R}$, of the class $\left[\mathcal{O}_{t r i v_{r}}\right]$ of the skyscraper sheaf supported at the trivial local system, see Proposition 3.6. Conversely, we determine the inverse image under $\Theta_{R}$ of the constant function $1_{\text {Bun }_{r} X}$, see Proposition 3.3.

0.5. To wrap up this paper, we provide two natural extensions of our results. By construction the spherical Hall algebra $\mathbf{U}_{X}$ is generated by the locally constant functions on the Picard group Pic X, i.e., by the simplest cuspidal functions in rank one. We define the principal Hall algebra to be the subalgebra of $\mathbf{H}_{V e c(X)}$ generated by all functions on Pic X. We generalize the description given in Theorem 1 as a shuffle algebra to the principal Hall algebra. Note that unlike the spherical Hall algebra, the principal Hall algebra depends on more than just the Weil numbers of $X$ : it also depends on the group structure of Pic X. Finally, the spherical Hall algebra and the K-theoretic Hall algebra both admit natural extensions to arbitrary reductive groups. And in fact neither the statement nor the proof of the Langlands isomorphism (0.7) use any special feature of the groups $G L_{r}$. We sketch such a generalization here. 
0.6. The content of the paper is as follows. In Section 1 we recall the definition of the Hall algebra of $X$, and give the shuffle presentation of the spherical Hall algebra $\mathbf{U}_{X}$. Section 2 is devoted to the Hall algebra in the (equivariant) K-theory of the genus $g$ commuting varieties $C_{\mathfrak{g l}_{r}}$. In Section 3 we state and prove our Langlands isomorphism and give the two applications mentioned in 0.4. above. The extensions of our results to the principal Hall algebra and to other reductive groups are sketched in two appendices at the end of the paper.

\section{Hall algebras of Curves}

1.1. Let $X$ be a smooth connected projective curve of genus $g$ defined over some finite field $\mathbb{F}_{q}$. Let $\zeta_{X}(t) \in \mathbb{C}(t)$ be its zeta function, i.e.,

$$
\zeta_{X}(t)=\exp \left(\sum_{d \geqslant 1} \# X\left(\mathbb{F}_{q^{d}}\right) \frac{t^{d}}{d}\right) .
$$

It is well-known that

$$
\zeta_{X}(t)=\frac{\prod_{i=1}^{g}\left(1-\alpha_{i} t\right)\left(1-\bar{\alpha}_{i} t\right)}{(1-t)(1-q t)}
$$

where $\left\{\alpha_{1}, \bar{\alpha}_{1}, \ldots, \alpha_{g}, \bar{\alpha}_{g}\right\}$ are the Frobenius eigenvalues in $H^{1}\left(X_{\bar{F}_{q}}, \overline{\mathbb{Q}}_{l}\right)$. Recall that $\left|\alpha_{i}\right|=q^{1 / 2}$, i.e., $\alpha_{i} \bar{\alpha}_{i}=q$, for all $i=1, \ldots, g$.

1.2. Let $\operatorname{Coh}(X)$ be the category of coherent sheaves over $X$, and let $\operatorname{Tor}(X)$ be the full subcategory of $\operatorname{Coh}(X)$ consisting of torsion sheaves. We will be interested in the Hall algebra $\mathbf{H}_{X}$ of $\operatorname{Coh}(X)$. We briefly recall its definition here for the reader's convenience, and refer to [S1], especially Section 4.11 for its standard properties. Let $K_{0}^{\prime}(X)=\mathbb{Z}^{2}$ be the numerical Grothendieck group of $\operatorname{Coh}(X)$. The class of a sheaf $\mathcal{F}$ is $\left(r_{\mathcal{F}}, d_{\mathcal{F}}\right)$ where we have abbreviated $r_{\mathcal{F}}=\operatorname{rank}(\mathcal{F})$, $d_{\mathcal{F}}=\operatorname{deg}(\mathcal{F})$. The Euler form on $K_{0}^{\prime}(X)$ is given by

$$
\langle\mathcal{F}, \mathcal{G}\rangle=\operatorname{dim} \operatorname{Hom}(\mathcal{F}, \mathcal{G})-\operatorname{dim} \operatorname{Ext}^{1}(\mathcal{F}, \mathcal{G})=(1-g) r_{\mathcal{F}} r_{\mathcal{G}}+\left|\begin{array}{ll}
r_{\mathcal{F}} & r_{\mathcal{G}} \\
d_{\mathcal{F}} & d_{\mathcal{G}}
\end{array}\right| .
$$

Let $\mathcal{I}$ be the set of isomorphism classes of coherent sheaves over $X$. Let us choose a square root $v$ of $q^{-1}$. As a vector space we have

$$
\mathbf{H}_{X}=\{f: \mathcal{I} \rightarrow \mathbb{C} ; \operatorname{supp}(f) \text { finite }\}=\bigoplus_{\mathcal{F} \in \mathcal{I}} \mathbb{C} 1_{\mathcal{F}}
$$

where $1_{\mathcal{F}}$ denotes the characteristic function of $\mathcal{F} \in \mathcal{I}$. The multiplication is defined as

$$
(f \cdot g)(\mathcal{R})=\sum_{\mathcal{N} \subseteq \mathcal{R}} v^{-\langle\mathcal{R} / \mathcal{N}, \mathcal{N}\rangle} f(\mathcal{R} / \mathcal{N}) g(\mathcal{N})
$$

and the comultiplication is

$$
\Delta(f)(\mathcal{M}, \mathcal{N})=\frac{v^{\langle\mathcal{M}, \mathcal{N}\rangle}}{\left|\operatorname{Ext}^{1}(\mathcal{M}, \mathcal{N})\right|} \sum_{\xi \in \operatorname{Ext}^{1}(\mathcal{M}, \mathcal{N})} f\left(\mathcal{X}_{\xi}\right)
$$

where $\mathcal{X}_{\xi}$ is the extension of $\mathcal{N}$ by $\mathcal{M}$ corresponding to $\xi$. Note that the coproduct takes values in a completion $\mathbf{H}_{X} \widehat{\otimes} \mathbf{H}_{X}$ of the tensor space $\mathbf{H}_{X} \otimes \mathbf{H}_{X}$ only, see e.g., (1.6). The Hall algebra $\mathbf{H}_{X}$ is $\mathbb{Z}^{2}$-graded, by the class in the Grothendieck group. We will sometimes write $\Delta_{\alpha, \beta}$ in order to specify the graded components of the coproduct. 
1.3. The triple $\left(\mathbf{H}_{X}, \cdot, \Delta\right)$ is not a (topological) bialgebra, but it becomes one if we suitably twist the coproduct. For this we introduce an extra subalgebra $\mathcal{K}=\mathbb{C}\left[\boldsymbol{\kappa}_{r, d}\right]$, with $(r, d) \in \mathbb{Z}^{2}$, and we define an extended Hall algebra $\widetilde{\mathbf{H}}_{X}=\mathbf{H}_{X} \otimes \mathcal{K}$ with relations

$$
\boldsymbol{\kappa}_{r, d} \boldsymbol{\kappa}_{s, l}=\boldsymbol{\kappa}_{r+s, d+l}, \quad \boldsymbol{\kappa}_{0,0}=1, \quad \boldsymbol{\kappa}_{r, d} 1_{\mathcal{M}} \boldsymbol{\kappa}_{r, d}^{-1}=v^{-2 r(1-g) r_{\mathcal{M}}} 1_{\mathcal{M}} .
$$

The new coproduct is given by the formulas

$$
\begin{gathered}
\widetilde{\Delta}\left(\boldsymbol{\kappa}_{r, d}\right)=\boldsymbol{\kappa}_{r, d} \otimes \boldsymbol{\kappa}_{r, d} \\
\widetilde{\Delta}(f)=\sum_{\mathcal{M}, \mathcal{N}} \Delta(f)(\mathcal{M}, \mathcal{N}) 1_{\mathcal{M}} \boldsymbol{\kappa}_{r_{\mathcal{N}}, d_{\mathcal{N}}} \otimes 1_{\mathcal{N}} .
\end{gathered}
$$

Then $\left(\widetilde{\mathbf{H}}_{X}, \cdot, \widetilde{\Delta}\right)$ is a topological bialgebra. Finally, let

$$
(,)_{G}: \widetilde{\mathbf{H}}_{X} \otimes \widetilde{\mathbf{H}}_{X} \rightarrow \mathbb{C}
$$

be Green's Hermitian scalar product defined by

$$
\left(1_{\mathcal{M}} \boldsymbol{\kappa}_{r, d}, 1_{\mathcal{N}} \boldsymbol{\kappa}_{s, l}\right)_{G}=\frac{\delta_{\mathcal{M}, \mathcal{N}}}{\# \operatorname{Aut}(\mathcal{M})} v^{-2(1-g) r s} .
$$

This scalar product satisfies the Hopf property, i.e., we have

$$
(a b, c)_{G}=(a \otimes b, \widetilde{\Delta}(c))_{G}, \quad a, b, c \in \widetilde{\mathbf{H}}_{X} .
$$

Observe that the restriction of $(,)_{G}$ to $\mathbf{H}_{X}$ is nondegenerate. The Hall algebras $\widetilde{\mathbf{H}}_{X}$ is also $\mathbb{Z}^{2}$-graded. We will write $\widetilde{\Delta}_{\alpha, \beta}$ in order to specify the graded components of the coproduct.

1.4. The category $\operatorname{Tor}(X)$ is a Serre subcategory of $\operatorname{Coh}(X)$. It therefore gives rise to a sub bialgebra $\widetilde{\mathbf{H}}_{T o r(X)}$ of $\widetilde{\mathbf{H}}_{X}$ defined by

$$
\widetilde{\mathbf{H}}_{\text {Tor }(X)}=\left(\bigoplus_{\mathcal{M} \in \operatorname{Tor}(X)} \mathbb{C} 1_{\mathcal{M}}\right) \otimes \mathcal{K} .
$$

The decomposition of $\operatorname{Tor}(X)$ over the points of $X$ gives rise to a similar decomposition at the level of Hall algebras

$$
\widetilde{\mathbf{H}}_{T o r(X)}=\left(\bigotimes_{x \in X} \mathbf{H}_{\operatorname{Tor}(X)_{x}}\right) \otimes \mathcal{K}
$$

where $\operatorname{Tor}(X)_{x}$ is the subcategory of torsion sheaves supported at $x \in X$. It is well-known that $\mathbf{H}_{\operatorname{Tor}(X)_{x}}$ is commutative and cocommutative : it is isomorphic to the classical Hall algebra. For $d \geqslant 1$ we set

$$
\mathbf{1}_{0, d}=\sum_{\substack{\mathcal{M} \in T o r(X) \\ \operatorname{deg}(\mathcal{M})=d}} 1_{\mathcal{M}}
$$

and we define elements $T_{0, d}, \theta_{0, d}$ of $\widetilde{\mathbf{H}}_{T o r(X)}$ through the relations

$$
1+\sum_{d \geqslant 1} \mathbf{1}_{0, d} s^{d}=\exp \left(\sum_{d} \frac{T_{0, d}}{[d]} s^{d}\right), \quad 1+\sum_{d \geqslant 1} \theta_{0, d} s^{d}=\exp \left(\left(v^{-1}-v\right) \sum_{d \geqslant 1} T_{0, d} s^{d}\right) .
$$

Here as usual $[n]=\left(v^{n}-v^{-n}\right) /\left(v-v^{-1}\right)$. We set also $\mathbf{1}_{0,0}=T_{0,0}=\theta_{0,0}=1$. The following lemma summarizes the properties of the elements $T_{0, d}, \theta_{0, d}$ which we will later need.

Lemma 1.1. The following hold for all $d \in \mathbb{N}$

(a) $\widetilde{\Delta}\left(T_{0, d}\right)=T_{0, d} \otimes 1+\boldsymbol{\kappa}_{0, d} \otimes T_{0, d}$,

(b) $\widetilde{\Delta}\left(\theta_{0, d}\right)=\sum_{l=0}^{d} \theta_{0, l} \boldsymbol{\kappa}_{0, d-l} \otimes \theta_{0, d-l}$,

(c) $\left(T_{0, d}, T_{0, d}\right)_{G}=\left(v^{d-1} \# X\left(\mathbb{F}_{q^{d}}\right)[d]\right) /(d q-d)$.

Proof. See [S1, Section 4.11]. 
The sets $\left\{\mathbf{1}_{0, d} ; d \in \mathbb{N}\right\},\left\{T_{0, d} ; d \in \mathbb{N}\right\}$, and $\left\{\theta_{0, d} ; d \in \mathbb{N}\right\}$ all generate the same subalgebra $\mathbf{U}_{X}^{0}$ of $\widetilde{\mathbf{H}}_{T o r(X)}$. It is known that

$$
\mathbf{U}_{X}^{0}=\mathbb{C}\left[\mathbf{1}_{0,1}, \mathbf{1}_{0,2}, \ldots\right]
$$

i.e., the commuting elements $\mathbf{1}_{0, d}$ for $d \geqslant 1$ are algebraically independent. Clearly, the same holds also for the collections of generators $\left\{\theta_{0, d}\right\},\left\{T_{0, d}\right\}$.

1.5. Now let $\operatorname{Vec}(X)$ be the exact subcategory of $\operatorname{Coh}(X)$ consisting of vector bundles. It gives rise to a subalgebra $\mathbf{H}_{V e c(X)}$ of $\mathbf{H}_{X}$. This subalgebra is, however, not stable under the coproduct $\Delta$. The multiplication map yields isomorphisms

$$
\mathbf{H}_{V e c(X)} \otimes \widetilde{\mathbf{H}}_{T o r(X)} \rightarrow \widetilde{\mathbf{H}}_{X}, \quad \mathbf{H}_{V e c(X)} \otimes \mathbf{H}_{T o r(X)} \rightarrow \mathbf{H}_{X}
$$

Indeed, if $\mathcal{V}$ is a vector bundle and $\mathcal{T}$ is a torsion sheaf then

$$
1_{\mathcal{V}} \cdot 1_{\mathcal{T}}=v^{-\langle\mathcal{V}, \mathcal{T}\rangle} 1_{\mathcal{V} \oplus \mathcal{T}}
$$

because $\operatorname{Ext}^{1}(\mathcal{V}, \mathcal{T})=0$ and because the subsheaf $\mathcal{T} \subset \mathcal{V} \oplus \mathcal{T}$ is canonically defined. The comultiplication provides an inverse to (1.1). To be more precise, given $\mathcal{V}, \mathcal{T}$ as above we have

$$
\Delta_{\overline{\mathcal{V}}, \overline{\mathcal{T}}}\left(1_{\mathcal{V} \oplus \mathcal{T}}\right)=v^{\langle\mathcal{V}, \mathcal{T}\rangle} 1_{\mathcal{V}} \otimes 1_{\mathcal{T}}
$$

This comes from the fact that $\mathcal{T}$ is the only torsion subsheaf of $\mathcal{V} \oplus \mathcal{T}$ of degree $d=\operatorname{deg}(\mathcal{T})$. We may use (1.1) to define a projection $\widetilde{\mathbf{H}}_{X} \rightarrow \mathbf{H}_{V e c(X)}$, which we will denote by $\omega$. It satisfies

$$
\omega\left(u_{v} u_{t} \boldsymbol{\kappa}_{r, d}\right)=\left\{\begin{array}{ll}
u_{v} & \text { if } u_{t}=1 \\
0 & \text { otherwise }
\end{array}, \quad u_{v} \in \mathbf{H}_{\operatorname{Vec}(X)}, \quad u_{t} \in \mathbf{H}_{T o r(X)}, \quad(r, d) \in \mathbb{Z}^{2} .\right.
$$

1.6. There is a natural action of the Hall algebra $\widetilde{\mathbf{H}}_{T o r(X)}$ on $\mathbf{H}_{V e c(X)}$ by means of the so-called Hecke operators, given by the formula

$$
\widetilde{\mathbf{H}}_{T o r(X)} \otimes \mathbf{H}_{V e c(X)} \rightarrow \mathbf{H}_{V e c(X)}, \quad u_{0} \otimes u \mapsto u_{0} \bullet w=\omega\left(u_{0} w\right),
$$

in other words, we have

$$
\left(u_{0} u_{0}^{\prime}\right) \bullet v=u_{0} \bullet\left(u_{0}^{\prime} \bullet v\right) .
$$

Proposition 1.2. The Hecke action on $\mathbf{H}_{V e c(X)}$ preserves each graded component $\mathbf{H}_{V e c(X)}[r]$, and we have $T_{0, d} \bullet w=\left[T_{0, d}, w\right]$ for $w \in \mathbf{H}_{V e c(X)}$ and $d>0$.

Proof. We have $T_{0, d} \bullet w=\omega\left(T_{0, d} w\right)=\omega\left(\left[T_{0, d}, w\right]\right)$. We claim that $\left[T_{0, d}, w\right]$ already belongs to $\mathbf{H}_{V e c(X)}$. Note that

$$
\begin{aligned}
& \widetilde{\Delta}\left(T_{0, d} w\right)=\left(T_{0, d} \otimes 1+\boldsymbol{\kappa}_{0, d} \otimes T_{0, d}\right) \widetilde{\Delta}(w), \\
& \widetilde{\Delta}\left(w T_{0, d}\right)=\widetilde{\Delta}(w)\left(T_{0, d} \otimes 1+\boldsymbol{\kappa}_{0, d} \otimes T_{0, d}\right), \\
& \widetilde{\Delta}(w) \in \widetilde{\mathbf{H}}_{X} \widehat{\otimes} \mathbf{H}_{V e c(X)} .
\end{aligned}
$$

Therefore $\widetilde{\Delta}\left(\left[T_{0, d}, w\right]\right)=\left[\widetilde{\Delta}\left(T_{0, d}\right), \widetilde{\Delta}(w)\right]$ has no component in $\mathbf{H}_{V e c(X)} \mathcal{K} \otimes \mathbf{H}_{T o r(X)}$. Hence (1.2) implies that $\left[T_{0, d}, w\right]$ belongs to $\mathbf{H}_{V e c(X)}$. 
1.7. For $d \in \mathbb{Z}$ let $\operatorname{Pic}^{d}(X)$ be the set of line bundles over $X$ of degree $d$. Put

$$
\mathbf{1}_{1, d}^{\mathrm{ss}}=\mathbf{1}_{\operatorname{Pic}^{d}(X)}=\sum_{\mathcal{L} \in \operatorname{Pic}^{d}(X)} 1_{\mathcal{L}} .
$$

Let us denote by $\mathbf{U}_{X}^{>}$the subalgebra of $\mathbf{H}_{X}$ generated by $\left\{\mathbf{1}_{1, d}^{\mathbf{s s}} ; d \in \mathbb{Z}\right\}$. The spherical Hall algebra of $X$ is the subalgebra ${ }^{1} \mathbf{U}_{X}$ of $\mathbf{H}_{X}$ generated by $\mathbf{U}_{X}^{0}$ and $\mathbf{U}_{X}^{>}$. We also define $\widetilde{\mathbf{U}}_{X}$ as the subalgebra of $\widetilde{\mathbf{H}}_{X}$ generated by $\mathcal{K}$ and $\mathbf{U}_{X}$. It is known that $\widetilde{\mathbf{U}}_{X}$ is a topological sub bialgebra of $\widetilde{\mathbf{H}}_{X}$. Finally, set $\widetilde{\mathbf{U}}_{X}^{0}=\mathbf{U}_{X}^{0} \mathcal{K}$. The multiplication map gives an isomorphism $\mathbf{U}_{X}^{>} \otimes \widetilde{\mathbf{U}}_{X}^{0} \stackrel{\sim}{\longrightarrow} \widetilde{\mathbf{U}}_{X}$, see e.g., [SV1, Section 6]. The coproduct of the elements $\mathbf{1}_{1, d}^{\mathrm{ss}}$ can be explicitly computed, see e.g., [S1, Ex. 4.12],

$$
\widetilde{\Delta}\left(\mathbf{1}_{1, d}^{\mathbf{s s}}\right)=\mathbf{1}_{1, d}^{\mathbf{s s}} \otimes 1+\sum_{l \geqslant 0} \theta_{0, l} \boldsymbol{\kappa}_{1, d-l} \otimes \mathbf{1}_{1, d-l}^{\text {ss }} .
$$

1.8. Our aim for the rest of this first section is to give a presentation of the algebra $\mathbf{U}_{X}$. When $X$ is of genus 0 or 1 this was done explicitly in [K], [BS]. In higher genus, we will provide a more implicit presentation, which will however suffice for our purposes here. Our approach, which is based on the notion of shuffle algebras, see e.g., [R2], [FJMM], can be developed for the Hall algebra of a more or less arbitrary hereditary category. We begin with a couple of preliminary lemmas.

Lemma 1.3. We have

$$
\left[T_{0, d}, \mathbf{1}_{1, l}^{s s}\right]=\frac{v^{d} \# X\left(\mathbb{F}_{q^{d}}\right)[d]}{d} \mathbf{1}_{1, l+d}^{s s}, \quad l \in \mathbb{Z}, \quad d>0 .
$$

Proof. The proof is very close to [BS, Lemma 4.12], see also [SV1, Theorem 6.3]. Because the left hand side is an element of $\mathbf{U}_{X}$, we may write

$$
\left[T_{0, d}, \mathbf{1}_{1, l}^{\mathbf{s s}}\right]=\sum_{n} \mathbf{1}_{1, n}^{\mathbf{s s}} u_{n}, \quad u_{n} \in \mathbf{U}_{\operatorname{Tor}(X)}^{0} .
$$

But because $\left[T_{0, d}, \mathbf{1}_{1, l}^{\mathrm{ss}}\right]=T_{0, d} \bullet \mathbf{1}_{1, l}^{\mathrm{ss}} \in \mathbf{H}_{V e c(X)}$ we must have in fact

$$
\left[T_{0, d}, \mathbf{1}_{1, l}^{\mathbf{s s}}\right]=c_{l, d} \mathbf{1}_{1, l+d}^{\mathbf{s s}}, \quad c_{l, d} \in \mathbb{C} .
$$

For this, we write

$$
\begin{aligned}
c_{l, d}\left(\mathbf{1}_{1, l+d}^{\mathbf{s s}}, \mathbf{1}_{1, l+d}^{\mathbf{s s}}\right)_{G} & =\left(\mathbf{1}_{1, l+d}^{\mathbf{s s}},\left[T_{0, d}, \mathbf{1}_{1, l}^{\mathbf{s s}}\right]\right)_{G} \\
& =\left(\mathbf{1}_{1, l+d}^{\mathbf{s s}}, T_{0, d}, \mathbf{1}_{1, l}^{\mathbf{s s}}\right)_{G} \\
& =\left(\widetilde{\Delta}\left(\mathbf{1}_{1, l+d}^{\mathbf{s s}}\right), T_{0, d} \otimes \mathbf{1}_{1, l}^{\mathbf{s s}}\right)_{G} \\
& =\left(\theta_{0, d} \boldsymbol{\kappa}_{1, l}, T_{0, d}\right)_{G}\left(\mathbf{1}_{1, l}^{\mathbf{s s}}, \mathbf{1}_{1, l}^{\mathbf{s s}}\right)_{G}
\end{aligned}
$$

and hence $c_{l, d}=\left(\theta_{0, d}, T_{0, d}\right)_{G}$. Developing $\theta_{0, d}$ in terms of the $T_{0, n}$ 's, using the Hopf property of $(,)_{G}$ and Lemma 1.1 a) we obtain

$$
\left(\theta_{0, d}, T_{0, d}\right)_{G}=\left(v^{-1}-v\right)\left(T_{0, d}, T_{0, d}\right)_{G}=\frac{v^{d} \# X\left(\mathbb{F}_{q^{d}}\right)[d]}{d}
$$

as wanted.

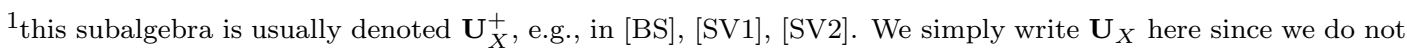
consider any Drinfeld double.
} 
Corollary 1.4. Define complex numbers $\xi_{k}, k \geqslant 0$ by $\omega\left(\theta_{0, k} \mathbf{1}_{1, l}^{s s}\right)=\xi_{k} \mathbf{1}_{1, l+k}^{s s}$. Then we have

(a) for $l \in \mathbb{Z}$ and $d \geqslant 0$,

$$
\theta_{0, d} \mathbf{1}_{1, l}^{s s}=\sum_{n=0}^{d} \xi_{n} \mathbf{1}_{1, l+n}^{s s} \theta_{0, d-n}
$$

(b) as a series in $\mathbb{C}[[s]]$ we have

$$
\sum_{d \geqslant 0} \xi_{d} s^{d}=\frac{\zeta_{X}(s)}{\zeta_{X}\left(q^{-1} s\right)}
$$

Proof. Statement (a) is a consequence of Lemma 1.3 and the definition of $\theta_{0, d}$. We prove $(b)$. By Lemma 1.3 we have

$$
\omega\left(T_{0, d} \mathbf{1}_{1, l}^{\mathbf{s s}}\right)=c_{d} \mathbf{1}_{1, l+d}^{\mathbf{s s}}, \quad c_{d}=v^{d} \# X\left(\mathbb{F}_{q^{d}}\right)[d] / d .
$$

It follows that $\omega\left(T_{0, d_{1}} \cdots T_{0, d_{r}} \mathbf{1}_{1, l}^{\mathbf{s s}}\right)=c_{d_{1}} \cdots c_{d_{r}} \mathbf{1}_{1, l+d_{1}+\cdots+d_{r}}^{\text {ss }}$. Now, we have

$$
\sum_{d \geqslant 0} \xi_{d} s^{d}=\exp \left(\left(v^{-1}-v\right) \sum_{d \geqslant 1} c_{d} s^{d}\right)=\exp \left(\sum_{d \geqslant 1} \# X\left(\mathbb{F}_{q^{d}}\right)\left(1-v^{2 d}\right) \frac{s^{d}}{d}\right)=\frac{\zeta_{X}(s)}{\zeta_{X}\left(q^{-1} s\right)} .
$$

1.9. In order to give presentations of $\mathbf{U}_{X}$ and $\mathbf{U}_{X}^{>}$we introduce the so-called constant term map. For $r \geqslant 1$ we set

$$
J_{r}: \mathbf{U}_{X}^{>}[r] \rightarrow \mathbf{U}_{X}^{>}[1] \widehat{\otimes} \cdots \widehat{\otimes} \mathbf{U}_{X}^{>}[1], \quad u \mapsto(\omega \otimes \cdots \otimes \omega) \Delta_{1, \ldots, 1}(u)
$$

and denote by $J: \mathbf{U}_{X}^{>} \rightarrow \bigoplus_{r}\left(\mathbf{U}_{X}^{>}[1]\right)^{\widehat{\otimes} r}$ the sum of the maps $J_{r}$. Writing

$$
J(u)=\sum_{\mathcal{L}_{1}, \ldots, \mathcal{L}_{r}} u\left(\mathcal{L}_{1}, \ldots, \mathcal{L}_{r}\right) 1_{\mathcal{L}_{1}} \otimes \cdots \otimes 1_{\mathcal{L}_{r}},
$$

we have

$$
u\left(\mathcal{L}_{1}, \ldots, \mathcal{L}_{r}\right)=\frac{1}{(q-1)^{r}}\left(J(u), 1_{\mathcal{L}_{1}} \otimes \cdots \otimes 1_{\mathcal{L}_{r}}\right)_{G}=\frac{1}{(q-1)^{r}}\left(u, 1_{\mathcal{L}_{1}} \cdots 1_{\mathcal{L}_{r}}\right)_{G}
$$

which coincides with the standard notion of constant term in the theory of automorphic forms (up to the factor $(q-1)^{-r}$ ), see $[\mathrm{K}]$. Observe that because $J_{r}$ lands in $\left(\mathbf{U}_{X}^{>}[1]\right)^{\widehat{\otimes} r}$ and $\mathbf{U}_{X}^{>}[1]=$ $\bigoplus_{d} \mathbf{1}_{1, d}^{\text {ss }}$, the function $u\left(\mathcal{L}_{1}, \ldots, \mathcal{L}_{r}\right)$ only depends on the respective degrees $d_{1}, \ldots, d_{r}$ of the line bundles $\mathcal{L}_{1}, \ldots, \mathcal{L}_{r}$.

Lemma 1.5. The constant term map $J: \mathbf{U}_{X}^{>} \rightarrow \bigoplus_{r}\left(\mathbf{U}_{X}^{>}[1]\right)^{\widehat{\otimes} r}$ is injective.

Proof. Let $u \in \mathbf{U}_{X}^{>}[r]$ be in the kernel of $J_{r}$. Then by (1.5) we have $\left(u, 1_{\mathcal{L}_{1}} \cdots 1_{\mathcal{L}_{r}}\right)_{G}=0$ for all $r$-tuples of line bundles $\left(\mathcal{L}_{1}, \ldots, \mathcal{L}_{r}\right)$. In particular,

$$
\left(u, \mathbf{1}_{1, d_{1}}^{\mathrm{ss}} \cdots \mathbf{1}_{1, d_{r}}^{\mathrm{ss}}\right)_{G}=0, \quad\left(d_{1}, \ldots, d_{r}\right) \in \mathbb{Z}^{r} .
$$

By construction $\mathbf{U}_{X}^{>}$is generated by the elements $\mathbf{1}_{1, d}^{\text {ss }}$ hence $\left(u, \mathbf{U}_{X}^{>}\right)_{G}=0$. But the restriction of $(,)_{G}$ to $\mathbf{U}_{X}^{>}$is known to be nondegenerate, see e.g., [S1, Thm. 4.52]. Therefore $u=0$ and $J_{r}$ is injective as wanted.

The objective is now to determine as precisely as possible the image of $J$ and to write the product and coproduct structure of $\mathbf{U}_{X}^{>}$in terms of $\bigoplus_{r}\left(\mathbf{U}_{X}^{>}[1]\right)^{\widehat{\otimes} r}$. For this it will be convenient to identify $\mathbf{U}_{X}^{>}[1]$ with $\mathbb{C}\left[x^{ \pm 1}\right]$ via the assignment

$$
\mathbf{1}_{1, d}^{\mathrm{ss}} \mapsto x^{d}, \quad d \in \mathbb{Z} .
$$


Thus we have

$$
\begin{aligned}
\left(\mathbf{U}_{X}^{>}[1]\right)^{\otimes r} & \simeq \mathbb{C}\left[x_{1}^{ \pm 1}, \ldots, x_{r}^{ \pm 1}\right], \\
\left(\mathbf{U}_{X}^{>}[1]\right)^{\otimes r} & \simeq \mathbb{C}\left[x_{1}^{ \pm 1}, \ldots, x_{r}^{ \pm 1}\right]\left[\left[x_{1} / x_{2}, \ldots, x_{r-1} / x_{r}\right]\right] .
\end{aligned}
$$

As it turns out, the (co)algebra structure of $\mathbf{U}_{X}^{>}$may be extended to the whole of $\bigoplus_{r}\left(\mathbf{U}_{X}^{>}[1]\right)^{\widehat{\otimes} r}$, where it is nicely expressed as a shuffle algebra. Before writing the definition of these shuffles algebras, we begin with a few notations. Let $\mathfrak{S}_{r}$ be the group of permutations of $\{1, \ldots, r\}$. If $w \in$ $\mathfrak{S}_{r}$ and $P\left(z_{1}, \ldots, z_{r}\right)$ a function in $r$ variables then we set $w P\left(z_{1}, \ldots, z_{r}\right)=P\left(z_{w(1)}, \ldots, z_{w(r)}\right)$. Let

$$
S h_{r, s}=\left\{w \in \mathfrak{S}_{r+s} ; w(i)<w(j) \text { if } 1 \leqslant i<j \leqslant r \text { or } r<i<j \leqslant r+s\right\}
$$

be the set of $(r, s)$-shuffles, i.e., the set of minimal lenght representatives of the left cosets in $\mathfrak{S}_{r+s} / \mathfrak{S}_{r} \times \mathfrak{S}_{s}$. Write

$$
I_{w}=\left\{(i, j) ; 1 \leqslant i<j \leqslant r+s, w^{-1}(i)>r \geqslant w^{-1}(j)\right\}, \quad w \in S h_{r, s} .
$$

Finally, let $h(z) \in \mathbb{C}(z)$ be a fixed rational function. We define an associative algebra $\mathbf{F}_{h(z)}$ as follows. As a vector space

$$
\mathbf{F}_{h(z)}=\bigoplus_{r} \mathbb{C}\left[x_{1}^{ \pm 1}, \ldots, x_{r}^{ \pm 1}\right]\left[\left[x_{1} / x_{2}, \ldots, x_{r-1} / x_{r}\right]\right],
$$

and the multiplication is given by

$$
P\left(x_{1}, \ldots, x_{r}\right) \star Q\left(x_{1}, \ldots, x_{s}\right)=\sum_{\sigma \in S h_{r, s}} h\left(x_{1}, \ldots, x_{r+s}\right) \sigma\left(P\left(x_{1}, \ldots, x_{r}\right) Q\left(x_{r+1}, \ldots, x_{r+s}\right)\right) .
$$

Here

$$
h_{\sigma}\left(x_{1}, \ldots, x_{r+s}\right)=\prod_{(i, j) \in I_{\sigma}} h\left(x_{i} / x_{j}\right),
$$

and the rational function $h\left(x_{i} / x_{j}\right)$ is developed as a Laurent series in $x_{1} / x_{2}, \ldots, x_{r-1} / x_{r}$. Note that the product (1.7) is well-defined, i.e., it lands in the right completion, because the sum ranges over $(r, s)$-shuffles. We equip also $\mathbf{F}_{h(z)}$ with a coproduct $\Delta: \mathbf{F}_{h(z)} \rightarrow \mathbf{F}_{h(z)} \widehat{\otimes} \mathbf{F}_{(h(z)}$ defined by

$$
\Delta_{s, t}\left(x_{1}^{i_{1}} \cdots x_{r}^{i_{r}}\right)=x_{1}^{i_{1}} \cdots x_{s}^{i_{s}} \otimes x_{1}^{i_{s+1}} \cdots x_{t}^{i_{s+t}}, \quad \Delta=\bigoplus_{r=s+t} \Delta_{s, t} .
$$

The subspace $\mathbf{F}_{h(z)}^{r a t}$ of $\mathbf{F}_{h(z)}$ consisting of Laurent series which are expansions of rational functions forms a subalgebra, which is moreover stable under the coproduct. We are ready to give a first description of $\mathbf{U}_{X}^{>}$as a shuffle algebra. Recall that we have identified $\bigoplus_{r}\left(\mathbf{U}_{X}^{>}[1]\right)^{\widehat{\otimes} r}$ with the vector space $\mathbf{F}_{h(z)}$ via (1.6).

Proposition 1.6. Set

$$
h_{X}(z)=q^{1-g} \zeta_{X}(z) / \zeta_{X}\left(q^{-1} z\right) .
$$

The constant term map $J: \mathbf{U}_{X}^{>} \rightarrow \mathbf{F}_{h_{X}(z)}$ is an algebra morphism such that

$$
\left(J_{s} \otimes J_{t}\right) \circ(\omega \otimes \omega) \circ \Delta_{s, t}=\Delta_{s, t} \circ J_{r}, \quad r=s+t .
$$

Proof. Because $\mathbf{U}_{X}^{>}$is generated in degree one, to show that $J$ is a algebra homomorphism it is enough to prove that for $\left(d_{1}, \ldots, d_{r}\right) \in \mathbb{Z}^{r}$ we have

$$
J_{r}\left(\mathbf{1}_{1, d_{1}}^{\mathrm{ss}} \cdots \mathbf{1}_{1, d_{r}}^{\mathrm{ss}}\right)=J_{1}\left(\mathbf{1}_{1, d_{1}}^{\mathrm{ss}}\right) \star \cdots \star J_{1}\left(\mathbf{1}_{1, d_{r}}^{\mathrm{ss}}\right)=x_{1}^{d_{1}} \star \cdots \star x_{1}^{d_{r}} .
$$

We will do this by computing the left hand side explicitly. We have

$$
\begin{aligned}
J_{r}\left(\mathbf{1}_{1, d_{1}}^{\mathrm{ss}} \cdots \mathbf{1}_{1, d_{r}}^{\mathrm{ss}}\right) & =\omega^{\otimes r} \widetilde{\Delta}_{\left(1^{r}\right)}\left(\mathbf{1}_{1, d_{1}}^{\mathrm{ss}} \cdots \mathbf{1}_{1, d_{r}}^{\mathrm{ss}}\right) \\
& =\omega^{\otimes r} \sum_{\sigma \in \mathfrak{S}_{r}} \widetilde{\Delta}_{\delta_{\sigma(1)}}\left(\mathbf{1}_{1, d_{1}}^{\mathrm{ss}}\right) \cdots \widetilde{\Delta}_{\delta_{\sigma(r)}}\left(\mathbf{1}_{1, d_{r}}^{\mathrm{ss}}\right),
\end{aligned}
$$


where by definition $\left(\delta_{1}, \cdots, \delta_{r}\right)$ is the standard basis of $\mathbb{Z}^{r}$. In the above, we have made use of the fact that $\widetilde{\Delta}$ is a morphism of algebras. Set $\theta_{d}=\theta_{0, d}$ in an effort to unburden the notation. Using (1.4) we get

$$
\widetilde{\Delta}_{\delta_{k}}\left(\mathbf{1}_{1, l}^{\mathbf{s s}}\right)=\sum_{d_{1}, \ldots, d_{k-1} \geqslant 0} \theta_{d_{1}} \boldsymbol{\kappa}_{1, l-d_{1}} \otimes \cdots \otimes \theta_{d_{k-1}} \boldsymbol{\kappa}_{1, l-\sum_{j<k} d_{j}} \otimes \mathbf{1}_{1, l-\sum_{j<k} d_{j}}^{\mathbf{s s}} \otimes 1 \otimes \cdots \otimes 1
$$

In order to compute the projection to vector bundles $\omega^{\otimes r}$, we need to reorder all the products appearing in the tensor components on the right hand side of (1.11) and to put them in the normal form $\mathbf{U}_{X}^{>} \cdot \widetilde{\mathbf{U}}_{X}^{0}$, see (1.3). For this, note that Corollary 1.4 yields

$$
\begin{aligned}
\sum_{u \geqslant 0} \theta_{u} \boldsymbol{\kappa}_{1, p-u} \mathbf{1}_{1, k}^{\mathbf{s s}} \otimes \mathbf{1}_{1, l-u}^{\mathbf{s s}} & =q^{1-g} \sum_{u \geqslant 0} \theta_{u} \mathbf{1}_{1, k}^{\mathbf{s s}} \boldsymbol{\kappa}_{p-u} \otimes \mathbf{1}_{1, l-u}^{\mathbf{s s}} \\
& =q^{1-g} \sum_{u \geqslant 0} \sum_{v=0}^{u} \xi_{v} \mathbf{1}_{k+v}^{\mathbf{s s}} \theta_{u-v} \boldsymbol{\kappa}_{1, p-u} \otimes \mathbf{1}_{1, l-u}^{s s} \\
& =q^{1-g} \sum_{v \geqslant 0} \sum_{w \geqslant 0} \xi_{v} \mathbf{1}_{k+v}^{\mathbf{s s}} \theta_{w} \boldsymbol{\kappa}_{1, p-v-w} \otimes \mathbf{1}_{1, l-v-w}^{\mathbf{s s}}
\end{aligned}
$$

Let us introduce the automorphism $\gamma$ of $\mathbf{U}_{X}^{>}[1]$ defined by $\gamma\left(\mathbf{1}_{1, n}^{\mathbf{s s}}\right)=\mathbf{1}_{1, n+1}^{\mathbf{s s}}$, and let us denote by $\gamma_{i}$ the operator $\gamma$ acting on the $i$ th component of the tensor product. Using this and Corollary 1.4 we may rewrite the right hand side of (1.13) as

$$
\begin{aligned}
q^{1-g} \sum_{v \geqslant 0} \xi_{v}\left(\gamma_{1} \gamma_{2}^{-1} \boldsymbol{\kappa}_{0,-1}\right)^{v} \sum_{w} \mathbf{1}_{k+v}^{\mathbf{s s}} \theta_{w} \boldsymbol{\kappa}_{1, p-w} \otimes \mathbf{1}_{1, l-w}^{\mathbf{s s}}= \\
=h_{X}\left(\gamma_{1} \gamma_{2}^{-1} \boldsymbol{\kappa}_{0,-1}\right) \sum_{w} \mathbf{1}_{k+v}^{\mathbf{s s}} \theta_{w} \boldsymbol{\kappa}_{1, p-w} \otimes \mathbf{1}_{1, l-w}^{\mathbf{s s}}
\end{aligned}
$$

For $\sigma \in \mathfrak{S}_{r}$ there is one contribution of the form (1.14) in (1.11) for each inversion $(i, j)$ of $\sigma$. Note that $\boldsymbol{\kappa}_{0,1}$ is central and we have $\omega\left(u \boldsymbol{\kappa}_{0,1}\right)=\omega(u)$ for all $u$ so that we may discard it. Thus all together we get

$$
J_{r}\left(\mathbf{1}_{1, d_{1}}^{\text {ss }} \cdots \mathbf{1}_{1, d_{r}}^{\text {ss }}\right)=\sum_{\sigma \in \mathfrak{S}_{r}} \prod_{(i, j) \in I_{\sigma}} h_{X}\left(\gamma_{i} \gamma_{j}^{-1}\right) \sigma\left(\mathbf{1}_{1, d_{1}}^{\text {ss }} \otimes \cdots \otimes \mathbf{1}_{1, d_{r}}^{\text {ss }}\right) .
$$

Observe that after the identification (1.6) with Laurent series, the operator $\gamma_{i}$ simply becomes the operator of multiplication by $x_{i}$. Hence we may write

$$
\begin{aligned}
J_{r}\left(\mathbf{1}_{1, d_{1}}^{\mathbf{s s}} \cdots \mathbf{1}_{1, d_{r}}^{\mathbf{s s}}\right) & =\sum_{\sigma \in \mathfrak{S}_{r}} \prod_{(i, j) \in I_{\sigma}} h_{X}\left(x_{i} / x_{j}\right) \sigma\left(x_{1}^{d_{1}} \cdots x_{r}^{d_{r}}\right) \\
& =x_{1}^{d_{1}} \star \cdots \star x_{1}^{d_{r}}
\end{aligned}
$$

as wanted. This proves that $J$ is a morphism of algebras. The statement regarding the coproduct is a direct consequence of the easily checked relation

$$
\omega^{\otimes r} \circ \widetilde{\Delta}_{\left(1^{r}\right)}=\left(\omega^{\otimes s} \otimes \omega^{\otimes t}\right) \circ \widetilde{\Delta}_{\left(1^{s}\right),\left(1^{t}\right)} \circ(\omega \otimes \omega) \circ \widetilde{\Delta}_{s, t} .
$$

By Proposition 1.6 above, $\mathbf{U}_{X}^{>}$is isomorphic to the subalgebra $\mathbf{S}_{h_{X}(z)}$ of $\mathbf{F}_{h_{X}(z)}$ generated by the degree one component $\mathbf{F}_{h_{X}(z)}[1]=\mathbb{C}\left[x_{1}^{ \pm 1}\right]$. Observe that $\mathbf{S}_{h_{X}(z)} \subset \mathbf{F}_{h_{X}(z)}^{r a t}$. 
Remark 1.7. The triple $\left(\mathbf{F}_{h(z)}, \star, \Delta\right)$ is not a bialgebra, just as it is wrong that $\left(\mathbf{U}_{X}^{>}, \cdot,(\omega \otimes \omega) \circ \Delta\right)$ is a bialgebra. Rather $\left(\mathbf{F}_{h(z)}, \star, \Delta\right)$ becomes a bialgebra after a suitable twist. This is very similar to the twist introduced by Lusztig in [L5]. Define a new multiplication $\star$ on $\mathbf{F}_{h(z)} \otimes \mathbf{F}_{h(z)}$ by introducing a correcting factor

$$
\left(a \otimes P\left(x_{1}, \ldots, x_{r}\right)\right) \star\left(Q\left(x_{1}, \ldots, x_{s}\right) \otimes b\right)=\left(a \star Q^{\prime}\left(x_{1}, \ldots, x_{s}\right)\right) \otimes\left(P^{\prime}\left(x_{1}, \ldots, x_{r}\right) \star b\right),
$$

where

$$
Q^{\prime}\left(x_{1}, \ldots, x_{s}\right) \otimes P^{\prime}\left(x_{1}, \ldots, x_{r}\right)=\left(\prod_{\substack{1 \leqslant i \leqslant r \\ 1 \leqslant j \leqslant s}} h_{X}\left(x_{j} \otimes x_{i}^{-1}\right)\right) Q\left(x_{1}, \ldots, x_{s}\right) \otimes P\left(x_{1}, \ldots, x_{r}\right) .
$$

It follows from (1.7) and (1.8) that $\left(\mathbf{F}_{h(z)}, \star, \Delta\right)$ is a twisted bialgebra, i.e., we have $\Delta(u \star v)=$ $\Delta(u) \star \Delta(v)$ for any $u, v \in \mathbf{F}_{h(z)}$. Moreover $\left(\mathbf{S}_{h(z)}, \star, \Delta\right)$ is a sub-bialgebra.

Remark 1.8. Formula (1.16) is essentially the Gindikin-Karpelevich identity, see e.g., [L1].

1.10. In this section we rephrase the results of Section 1.9 in more convenient terms. Namely, we identify $\mathbf{U}_{X}^{>}$with another shuffle algebra, but this time in a space of symmetric polynomials. This is precisely the setting of $[\mathrm{FO}]$, which we now explain. Let $g(z) \in \mathbb{C}(z)$ be a rational function. For $r \geqslant 1$ we put $g\left(x_{1}, \ldots, x_{r}\right)=\prod_{i<j} g\left(x_{i} / x_{j}\right)$. Let us denote by

$$
\text { Sym }_{r}: \mathbb{C}\left(x_{1}, \ldots, x_{r}\right) \rightarrow \mathbb{C}\left(x_{1}, \ldots, x_{r}\right)^{\mathfrak{S}_{r}}, \quad P\left(x_{1}, \ldots, x_{r}\right) \mapsto \sum_{w \in \mathfrak{S}_{r}} w P\left(x_{1}, \ldots, x_{r}\right)
$$

the standard symmetrization operator and let us consider the weighted symmetrization

$$
\Psi_{r}: \mathbb{C}\left[x_{1}^{ \pm 1}, \ldots, x_{r}^{ \pm r}\right] \rightarrow \mathbb{C}\left(x_{1}, \ldots, x_{r}\right)^{\mathfrak{S}_{r}}, \quad P\left(x_{1}, \ldots, x_{r}\right) \mapsto \operatorname{Sym}_{r}\left(g\left(x_{1}, \ldots, x_{r}\right) P\left(x_{1}, \ldots, x_{r}\right)\right) .
$$

Let $\mathbf{A}_{r}$ be the image of $\Psi_{r}$. There is a unique map $m_{r, s}: \mathbf{A}_{r} \otimes \mathbf{A}_{s} \rightarrow \mathbf{A}_{r+s}$ which makes the following diagram commute

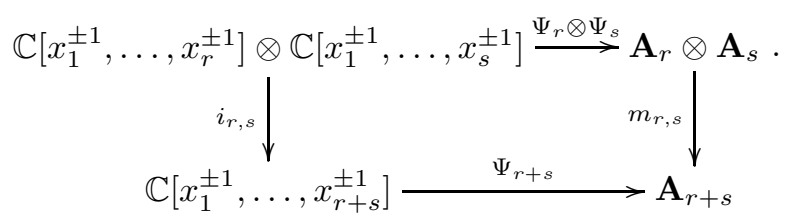

Here $i_{r, s}$ is the obvious isomorphism

$$
i_{r, s}\left(P\left(x_{1}, \ldots, x_{r}\right) \otimes Q\left(x_{1}, \ldots, x_{s}\right)\right)=P\left(x_{1}, \ldots, x_{r}\right) Q\left(x_{r+1}, \ldots, x_{r+s}\right) .
$$

The maps $m_{r, s}$ endow the space

$$
\mathbf{A}_{g(z)}=\mathbb{C} 1 \oplus \bigoplus_{r \geqslant 1} \mathbf{A}_{r}
$$

with the structure of an associative algebra whose product is given by

$$
P\left(x_{1}, \ldots, x_{r}\right) \star Q\left(x_{1}, \ldots, x_{s}\right)=\sum_{w \in S h_{r, s}} w\left(\prod_{\substack{1 \leqslant i \leqslant r \\ r+1 \leqslant j \leqslant r+s}} g\left(x_{i} / x_{j}\right) P\left(x_{1}, \ldots, x_{r}\right) Q\left(x_{r+1}, \ldots, x_{r+s}\right)\right) .
$$

Note that, by construction, the algebra $\mathbf{A}_{g(z)}$ is generated by the subspace $\mathbf{A}_{1}=\mathbb{C}\left[x_{1}^{ \pm 1}\right]$. As before, the shuffle product $m_{r, s}$ may be extended to the whole space $\bigoplus_{r} \mathbb{C}\left(x_{1}, \ldots, x_{r}\right)^{\mathfrak{S}_{r}}$.

Proposition 1.9. Let $g(z)$ be a rational function such that $h(z)=g\left(z^{-1}\right) / g(z)$. Then the assignment $x_{1}^{l} \mapsto x_{1}^{l}$ in degree one extends to an algebra isomorphism $\mathbf{S}_{h(z)} \rightarrow \mathbf{A}_{g(z)}$. 
Proof. Consider the transformation

$$
\Xi: \quad \mathbf{A}_{g(z)} \rightarrow \mathbf{F}_{h(z)}, \quad P\left(x_{1}, \ldots, x_{r}\right) \mapsto g^{-1}\left(x_{1}, \ldots, x_{r}\right) P\left(x_{1}, \ldots, x_{r}\right)
$$

Comparing the definitions of the shuffle products (1.7) and (1.19) one checks that $\Xi$ is an algebra embedding. It maps $\mathbf{A}_{g(z)}$ to $\mathbf{S}_{h(z)}$ since both are generated in degree one. The proposition follows.

Corollary 1.10. Let $g_{X}(z)$ be a rational function such that

$$
h_{X}(z)=g_{X}\left(z^{-1}\right) / g_{X}(z) .
$$

Then the map $\mathbf{1}_{1, l}^{s s} \mapsto x_{1}^{l}$ extends to an algebra isomorphism $\Upsilon_{X}: \mathbf{U}_{X}^{>} \rightarrow \mathbf{A}_{g_{X}(z)}$.

Using the functional equation for zeta functions

$$
\zeta_{X}\left(q^{-1} z\right)=z^{2(g-1)} q^{1-g} \zeta_{X}\left(z^{-1}\right)
$$

one checks that $z^{g-1} \zeta_{X}\left(z^{-1}\right)$ is a solution of (1.20). The same is also true of $z^{g-1} \zeta_{X}\left(z^{-1}\right) k(z)$ for any function $k(z)$ satisfying $k(z)=k\left(z^{-1}\right)$. It will actually be more convenient for us to set

$$
\begin{gathered}
\tilde{\zeta}_{X}(z)=\zeta_{X}(z)(1-q z)\left(1-q z^{-1}\right)=\frac{1-q z^{-1}}{1-z} \prod_{i=1}^{g}\left(1-\alpha_{i} z\right)\left(1-\bar{\alpha}_{i} z\right), \\
g_{X}(z)=z^{g-1} \tilde{\zeta}_{X}\left(z^{-1}\right) .
\end{gathered}
$$

From now on we fix the above choice for $g_{X}(z)$ and simply write $\mathbf{A}=\mathbf{A}_{g_{X}(z)}$. Thus we have an algebra isomorphism

$$
\Upsilon_{X}: \mathbf{U}_{X}^{>} \rightarrow \mathbf{A}
$$

Remark 1.11. The coproduct structure on $\mathbf{U}_{X}^{>}$may also be written down explicitly in terms of $\mathbf{A}$, but it is rather less pleasant than (1.8). We won't need this.

1.11. So far, we have only considered the spherical Hall algebra $\mathbf{U}_{X}$ and its vector bundle part $\mathbf{U}_{X}^{>}$for a fixed curve $X$ of genus $g$. However, these only depend on the zeta function of $X$. Equivalently, we may view $\mathbf{U}_{X}$ and $\mathbf{U}_{X}^{>}$as the specializations at $\left(\alpha_{1}, \bar{\alpha}_{1}, \ldots, \alpha_{g}, \bar{\alpha}_{g}\right)$ of a "universal" algebra which depends on the genus $g$ and on a point in the torus

$$
T_{a}=\left\{\left(\eta_{1}, \bar{\eta}_{1}, \ldots, \eta_{g}, \bar{\eta}_{g}\right) \in\left(\mathbb{C}^{\times}\right)^{2 g} ; \eta_{i} \bar{\eta}_{i}=\eta_{j} \bar{\eta}_{j}, \forall i, j\right\} \simeq\left(\mathbb{C}^{\times}\right)^{g+1} .
$$

Let

$$
R_{a}=R_{T_{a}}, \quad K_{a}=K_{T_{a}}
$$

be the complexified representation ring of $T_{a}$ and its fraction field. We define, using the shuffle presentation of Section 1.6, some $K_{a}$-algebras $\mathbf{U}_{K_{a}}^{>}=\mathbf{A}_{K_{a}}, \mathbf{U}_{K_{a}}, \ldots$ The bialgebra structure and Green's bilinear form both depend polynomially on $\left(\alpha_{1}, \bar{\alpha}_{1}, \ldots, \alpha_{g}, \bar{\alpha}_{g}\right)$ and may hence be defined over $K_{a}$. Let $\mathbf{A}_{R_{a}}$ be the $R_{a}$-subalgebra of $\mathbf{A}_{K_{a}}$ generated by $R_{a}\left[x_{1}^{ \pm 1}\right] \subset \mathbf{A}_{K_{a}}[1]$. By construction $\mathbf{A}_{R_{a}}$ is a torsion-free integral form of $\mathbf{A}_{K_{a}}$, in the sense that $\mathbf{A}_{R_{a}} \otimes_{R_{a}} K_{a}=\mathbf{A}_{K_{a}}$. Moreover there exists a natural specialization map

$$
\mathbf{A}_{R_{a}} \rightarrow \mathbf{U}_{X}^{>}, \quad x_{1}^{d} \mapsto \mathbf{1}_{1, d}^{\text {ss }}, \quad d \in \mathbb{Z},
$$

for a fixed curve $X$ of genus $g$. We'll write $\mathbf{U}_{R_{a}}^{>}=\mathbf{A}_{R_{a}}$ to emphasize this link with Hall algebras. 
1.12. In this paragraph we partially describe the image of the constant term map in rank $r$, i.e., we try to determine $\mathbf{A}_{r}$ inside the space of all symmetric rational functions. For this we consider the action of $\mathbf{U}_{X}^{0}$ on $\mathbf{U}_{X}^{>}$by means of the Hecke operators. Indeed, the presentation of $\mathbf{U}_{X}^{>}$as a shuffle algebra is particularly well suited to understand this action. Define algebra homomorphisms

$$
\pi_{r}: \mathbf{U}_{X}^{0} \rightarrow \mathbb{C}\left[x_{1}^{ \pm 1}, \ldots, x_{r}^{ \pm 1}\right]^{\mathfrak{S}_{r}}, \quad T_{0, d} \mapsto c_{d} p_{d}\left(x_{1}, \ldots, x_{r}\right)=c_{d}\left(\sum_{i} x_{i}^{d}\right), \quad d \geqslant 0
$$

where $c_{d}=v^{d} \# X\left(\mathbb{F}_{q^{d}}\right)[d] / d$. From Lemma 1.3 one deduces the following result.

Lemma 1.12. For any $r \geqslant 1$ the map $\Upsilon_{X}$ intertwines the Hecke action of $\mathbf{U}_{X}^{0}$ on $\mathbf{U}_{X}^{>}[r]$ with the natural action of $\mathbb{C}\left[x_{1}^{ \pm 1}, \ldots, x_{r}^{ \pm 1}\right]^{\mathfrak{S}_{r}}$ on $\mathbf{A}_{r}$, i.e., we have

$$
\Upsilon_{X}\left(u_{0} \bullet v\right)=\pi_{r}\left(u_{0}\right) \cdot \Upsilon_{X}(v), \quad u_{0} \in \mathbf{U}_{X}^{0}, \quad v \in \mathbf{U}_{X}^{>}[r]
$$

In other words, the $\mathbf{U}_{X}^{0}$-module structure of $\mathbf{U}_{X}^{>}[r]$ is reflected in the structure of $\Upsilon_{X}\left(\mathbf{U}_{X}^{>}[r]\right)=$ $\mathbf{A}_{r}$ as a $\mathbb{C}\left[x_{1}^{ \pm 1}, \ldots, x_{r}^{ \pm r}\right]^{\mathfrak{S}_{r}}$-module. In particular,

Corollary 1.13. The Hecke action of $\mathbf{U}_{X}^{0}$ on $\mathbf{U}_{X}^{>}$is torsion free.

The above definitions can be made for the Hall algebras $\mathbf{U}_{K_{a}}^{>}$over the field $K_{a}$ as well, see Section 1.11. The following proposition describes the structure of $\mathbf{U}_{K_{a}}^{>}[r]$ as a $K_{a}\left[x_{1}^{ \pm 1}, \ldots, x_{r}^{ \pm 1}\right]^{\mathfrak{S}_{r_{-}}}$ module. Set

$$
\Delta(x)=\prod_{i<j}\left(x_{i}-x_{j}\right)
$$

It is easy to see that $\mathbf{A}_{K_{a}, r}$ is an ideal of $K_{a}\left[x_{1}^{ \pm 1}, \ldots, x_{r}^{ \pm 1}\right]^{\mathfrak{S}_{r}}$.

Proposition 1.14. For $r \geqslant 1$ we have

$$
\Delta(x)^{r ! / 2} I_{r} \subseteq \mathbf{A}_{K_{a}, r} \subseteq I_{r}
$$

Here $I_{r} \subset K_{a}\left[x_{1}^{ \pm 1}, \ldots, x_{r}^{ \pm 1}\right]^{\mathfrak{S}_{r}}$ is the ideal sheaf of the reduced closed subvariety $Z_{r} \subset S^{r}\left(K_{a}^{*}\right)$ defined as

$$
Z_{r}=\bigcup_{\alpha} Z_{r}^{(\alpha)}, \quad Z_{r}^{(\alpha)}=\left\{\mathfrak{S}_{r}\left(x_{1}, \ldots, x_{r}\right) ; x_{1}=\alpha x_{2}, x_{2}=\bar{\alpha} x_{3}\right\}
$$

where $\alpha$ runs into $\left\{\alpha_{1}, \bar{\alpha}_{1}, \ldots, \alpha_{g}, \bar{\alpha}_{g}\right\}$.

Proof. Recall that $\mathbf{A}_{K_{a}, r}$ is the image of the operator $\Psi_{r}$ defined by

$$
\begin{aligned}
& \Psi_{r}\left(P\left(x_{1}, \ldots, x_{r}\right)\right)= \\
& \quad=\sum_{\sigma \in \mathfrak{S}_{r}} \sigma\left(\prod_{i<j}\left(\frac{x_{i}}{x_{j}}\right)^{g-1} \frac{\left(1-q^{-1} x_{j} / x_{i}\right) \prod_{l=1}^{g}\left(1-\alpha_{l} x_{j} / x_{i}\right)\left(1-\bar{\alpha}_{l} x_{j} / x_{i}\right)}{\left(1-x_{j} / x_{i}\right)} P\left(x_{1}, \ldots, x_{r}\right)\right) .
\end{aligned}
$$

We have

$$
\Psi_{r}\left(P\left(x_{1}, \ldots, x_{r}\right)\right)=\frac{\left(x_{1} \ldots x_{r}\right)^{2(1-g)}\left(-q^{r(r-1) / 2}\right)}{\Delta(x)} \sum_{\sigma}(-1)^{l(\sigma)} \sigma\left(\prod_{\substack{i<j \\ \gamma \in \Gamma}}\left(x_{i}-\gamma x_{j}\right) P\left(x_{1}, \ldots, x_{r}\right)\right),
$$

where $\Gamma=\left\{\alpha_{1}, \bar{\alpha}_{1}, \ldots, \alpha_{g}, \bar{\alpha}_{g}, q^{-1}\right\}$. Thus $\mathbf{A}_{K_{a}, r}=\operatorname{Im} \Psi_{r}^{\prime}$ with $\Psi_{r}^{\prime}$ defined by

$$
\Psi_{r}^{\prime}\left(P\left(x_{1}, \ldots, x_{r}\right)\right)=\frac{1}{\Delta(x)} \sum_{\sigma}(-1)^{l(\sigma)} \sigma\left(\prod_{\substack{i<j \\ \gamma \in \Gamma}}\left(x_{i}-\gamma x_{j}\right) P\left(x_{1}, \ldots, x_{r}\right)\right) .
$$


Now let $\alpha \in\left\{\alpha_{1}, \bar{\alpha}_{1}, \ldots, \alpha_{g}, \bar{\alpha}_{g}\right\}$. For any $\sigma \in \mathfrak{S}_{r}$ we have either $\sigma^{-1}(1)<\sigma^{-1}(2)$, or $\sigma^{-1}(2)<$ $\sigma^{-1}(3)$, or $\sigma^{-1}(3)<\sigma^{-1}(1)$. This means that for any $\sigma$ the element

$$
X_{\sigma}(x)=\sigma\left(\prod_{i<j, \gamma \in \Gamma}\left(x_{i}-\gamma x_{j}\right)\right)
$$

is divisible by $x_{1}-\alpha x_{2}$, by $x_{2}-\bar{\alpha} x_{3}$ or by $x_{3}-q^{-1} x_{1}$. Of course, the same holds if we replace $(1,2,3)$ by any other triplet of elements in $\{1, \ldots, r\}$. In all cases, $X_{\sigma}(x)$ belongs to the ideal sheaf of $Z_{r}^{(\alpha)}$. It follows that

$$
\Psi_{r}^{\prime}\left(P\left(x_{1}, \ldots, x_{r}\right)\right)=\Delta(x)^{-1} \sum_{\sigma}(-1)^{l(\sigma)} X_{\sigma}(x) \sigma P\left(x_{1}, \ldots, x_{r}\right)
$$

belongs to that ideal sheaf as well, for any polynomial $P\left(x_{1}, \ldots, x_{r}\right)$. We have proved that supp $\mathbf{A}_{K_{a}, r} \supset Z_{r}$, i.e., that $\mathbf{A}_{K_{a}, r} \subset I_{r}$.

We now prove the reverse inclusion. Let $\pi:\left(K_{a}^{*}\right)^{r} \rightarrow S^{r} K_{a}$ be the standard projection. It will be convenient to lift everything to $\left(K_{a}^{*}\right)^{r}$ via $\pi$. Put $\tilde{\mathbf{A}}_{K_{a}, r}=\mathbf{A}_{K_{a}, r} \otimes K_{a}\left[x_{1}^{ \pm 1}, \ldots, x_{r}^{ \pm 1}\right]$, where the tensor product is taken over $K_{a}\left[x_{1}^{ \pm 1}, \ldots, x_{r}^{ \pm 1}\right]^{\mathfrak{S}_{r}}$. This is an ideal of $K_{a}\left[x_{1}^{ \pm 1}, \ldots, \mathbf{x}_{r}^{ \pm 1}\right]$, and we have

$$
\mathbf{A}_{K_{a}, r}=\tilde{\mathbf{A}}_{K_{a}, r} \cap K_{a}\left[x_{1}^{ \pm 1}, \ldots, x_{r}^{ \pm 1}\right]^{\mathfrak{S}_{r}} .
$$

The space $K_{a}\left[x_{1}^{ \pm 1}, \ldots, x_{r}^{ \pm 1}\right]$, viewed as a $K_{a}\left[x_{1}^{ \pm 1}, \ldots, x_{r}^{ \pm 1}\right]^{\mathfrak{S}_{r}}$-module, is freely generated by the $r$ ! monomials $\left\{x_{1}^{n_{1}} \cdots x_{r}^{n_{r}} ; 0 \leqslant n_{i} \leqslant r-i\right\}$, see e.g., [L2, Lemma 7.6.1]. We deduce that $\mathbf{A}_{K_{a}, r}$ is generated over $K_{a}\left[x_{1}^{ \pm 1}, \ldots, x_{r}^{ \pm 1}\right]^{\mathfrak{S}_{r}}$ by the $r$ ! elements in

$$
\left\{\Psi^{\prime}\left(x_{1}^{n_{1}} \cdots x_{r}^{n_{r}}\right) ; 0 \leqslant n_{i} \leqslant r-i\right\}
$$

and thus that $\tilde{\mathbf{A}}_{K_{a}, r}$ is the ideal of $K_{a}\left[x_{1}^{ \pm 1}, \ldots, x_{r}^{ \pm 1}\right]$ generated by the set

$$
\left\{\Psi^{\prime}\left(x_{1}^{n_{1}} \cdots x_{r}^{n_{r}}\right) ; 0 \leqslant n_{i} \leqslant r-i\right\} .
$$

Consider the $r ! \times r$ ! square matrix $B=\left(b_{\sigma, I}\right)_{\sigma, I}$ where $\sigma \in \mathfrak{S}_{r}, I \in\left\{\left(n_{1}, \ldots, n_{r}\right) ; 0 \leqslant n_{i} \leqslant r-i\right\}$, with entries

$$
b_{\sigma, I}=\frac{1}{\Delta(x)}(-1)^{l(\sigma)} \sigma\left(x_{1}^{n_{1}} \cdots x_{r}^{n_{r}}\right) .
$$

Let $\Psi$ be the column vector with entries $\Psi_{r}^{\prime}\left(x_{1}^{n_{1}} \cdots x_{r}^{n_{r}}\right)$ for $\left(n_{1}, \ldots, n_{r}\right) \in I$ and likewise let $X$ be the column vector with entries $X_{\sigma}(x)$ for $\sigma \in \mathfrak{S}_{r}$, so that we have $\Psi=B \cdot X$.

Lemma 1.15. We have $\operatorname{det}(B)= \pm \Delta(x)^{-r ! / 2}$.

Proof. Left to the reader. The sign depends on the particular choice of ordering for the rows or columns of $B$.

Thus, the matrix $B$ has an inverse $B^{-1}$ whose entries belong to $\Delta(x)^{1-r ! / 2} K_{a}\left[x_{1}^{ \pm 1}, \ldots, x_{r}^{ \pm 1}\right]$. As a consequence, $X_{\sigma}(x)$ belongs to

$$
\Delta(x)^{1-r ! / 2} \sum_{n_{1}, \ldots, n_{r}} K_{a}\left[x_{1}^{ \pm 1}, \ldots, x_{r}^{ \pm 1}\right] \Psi_{r}^{\prime}\left(x_{1}^{n_{1}} \cdots x_{r}^{n_{r}}\right)=\Delta(x)^{1-r ! / 2} \tilde{\mathbf{A}}_{K_{a}, r} .
$$

Let $\tilde{J}_{r}$ be the ideal of $K_{a}\left[x_{1}^{ \pm 1}, \ldots, x_{r}^{ \pm 1}\right]$ generated by the $\left\{X_{\sigma}(x)\right\}_{\sigma}$. Let also

$$
\tilde{I}_{r}=I_{r} \otimes K_{a}\left[x_{1}^{ \pm 1}, \ldots, x_{r}^{ \pm 1}\right]
$$

be the (radical) ideal sheaf of $\pi^{-1}\left(Z_{r}\right)$. We will show that $\tilde{J}_{r}=\tilde{I}_{r}$ by checking that supp $\tilde{J}_{r}=$ $\pi^{-1}\left(Z_{r}\right)$ and that $\tilde{J}_{r}$ is radical. Indeed, we have

$$
\text { supp } \tilde{J}_{r}=\bigcap_{\sigma} \operatorname{supp} X_{\sigma}(x) .
$$

This last intersection is precisely given by the equations (1.25) as we now show. Let $\left(z_{1}, \ldots, z_{r}\right) \in$ $\left(K_{a}^{*}\right)^{r}$ satisfy $X_{\sigma}\left(z_{1}, \ldots, z_{r}\right)=0$ for all $\sigma$. This means that for every $\sigma$ there exists a pair $(i, j)$ and $\gamma \in \Gamma$ such that $\sigma(i)<\sigma(j)$ and $z_{i}=\gamma z_{j}$. Let us draw an arrow $i \rightarrow j$ if $z_{i} / z_{j} \in \Gamma$. If 
the graph on $\{1, \ldots, r\}$ contains no oriented cycles then we can find a permutation $\sigma$ satisfying $i \rightarrow j \Rightarrow \sigma(i)>\sigma(j)$, and this $\sigma$ violates the above condition. Hence there exists a cycle $i_{1} \rightarrow i_{2} \rightarrow \cdots \rightarrow i_{l} \rightarrow i_{1}$. Note that we have $\frac{z_{i_{1}}}{z_{i_{2}}} \cdots \frac{z_{i_{l}}}{z_{i_{1}}}=1$. A sequence of elements of $\Gamma$ which multiplies to one necessarily contains a subsequence $\left(\alpha, q^{-1}\right)$. But this means that there are three indices $i, j, k$ such that $z_{i}=\bar{\alpha} z_{j}, z_{j}=\alpha z_{k}$ (recall that $\alpha \bar{\alpha}=q$ ). In other words, we have

$$
\left(z_{1}, \ldots, z_{r}\right) \in \pi^{-1}\left(Z_{r}\right), \quad \bigcap_{\sigma} \operatorname{supp} X_{\sigma}(x)=\pi^{-1}\left(Z_{r}\right)
$$

as wanted. We now check that $\tilde{J}_{r}$ is a radical ideal. Set $S_{r}=K_{a}\left[x_{1}^{ \pm 1}, \ldots, x_{r}^{ \pm 1}\right] / \tilde{J}_{r}$. We have to prove that no element of $S_{r}$ is nilpotent. For this, it is enough to check that for each irreducible component $C$ of $\pi^{-1}\left(Z_{r}\right)$ there exists a point $z \in C$ for which the localization $S_{r, z}$ of $S_{r}$ at $z$ has no nilpotent. We have

$$
\pi^{-1}\left(Z_{r}\right)=\bigcup_{(\underline{i}, \alpha)} \tilde{Z}_{r}^{(\underline{i}, \alpha)}, \quad \tilde{Z}_{r}^{(\underline{i}, \alpha)}=\left\{\left(x_{1}, \ldots, x_{r}\right) ; x_{i_{1}}=\alpha x_{i_{2}}, x_{i_{2}}=\bar{\alpha} x_{i_{3}}\right\},
$$

where $\underline{i}=\left(i_{1}, i_{2}, i_{3}\right)$ runs among all triplets of elements of $\{1, \ldots, r\}$ and $\alpha \in\left\{\alpha_{1}, \bar{\alpha}_{1}, \ldots, \alpha_{g} \bar{\alpha}_{g}\right\}$. For a given $(\underline{i}, \alpha)$ as above let us pick a generic point $z=\left(z_{1}, \ldots, z_{r}\right)$ of $\tilde{Z}_{r}^{(\underline{i}, \alpha)}$. Hence $z$ satisfies

$$
z_{i_{1}}=\alpha z_{i_{2}}, \quad z_{i_{2}}=\bar{\alpha} z_{i_{3}}, \quad z_{i_{3}}=q^{-1} z_{i_{1}}
$$

but $z_{i} / z_{j} \notin \Gamma$ for any other value of $(i, j)$. The functions $\left(x_{i}-\gamma x_{j}\right)$ are therefore invertible in $S_{r, z}$ except for the three values $(i, j, \gamma)$ above. We may choose a permutation $\sigma \in \mathfrak{S}_{r}$ for which $\sigma^{-1}\left(i_{3}\right)<\sigma^{-1}\left(i_{1}\right)<\sigma^{-1}\left(i_{2}\right)$. Then we have

$$
X_{\sigma}(x)=\left(x_{i_{1}}-\alpha x_{i_{2}}\right) Y(x)
$$

for some polynomial $Y(x)$ which is invertible in $S_{r, z}$. But this means that $x_{i_{1}}-\alpha x_{i_{2}}=0$ in $S_{r, z}$. A similar argument shows that $x_{i_{2}}-\bar{\alpha} x_{i_{3}}=x_{i_{3}}-q^{-1} x_{i_{1}}=0$ in $S_{r, z}$ as well. We deduce that

$$
S_{r, z}=K_{a}\left[x_{1}^{ \pm 1}, \ldots, x_{r}^{ \pm 1}\right]_{z} /\left\langle x_{i_{1}}-\alpha x_{i_{2}}, x_{i_{2}}-\bar{\alpha} x_{i_{3}}\right\rangle .
$$

In particular $S_{r, z}$ has no nilpotent element. We have proved that $\tilde{J}_{r}$ is radical. We have shown that

$$
\tilde{\mathbf{A}}_{K_{a}, r} \supset \Delta(x)^{r ! / 2-1} \tilde{I}_{r} .
$$

Taking the intersection with $K_{a}\left[x_{1}^{ \pm 1}, \ldots, x_{r}^{ \pm 1}\right]^{\mathfrak{S}_{r}}$ yields the desired inclusion. This finishes the proof of Proposition 1.14.

Remark 1.16. (a) Equations (1.25) are dubbed wheel conditions in [FJMM], in the general context of Feigin-Odesskii algebras. A weaker form of Proposition 1.14 appears in [FT] for $g=1$. Note that $Z_{r}$ is the image under the natural map $\left(K_{a}^{*}\right)^{r} \rightarrow S^{r} K_{a}^{*}$ of a union of $2 g$ codimension two subspaces. Observe also that $Z_{r}$ is empty for $r=2$ but not for any $r>2$. As a consequence $\mathbf{A}_{K_{a}, r}$ is $n o t$ free over $K_{a}\left[x_{1}^{ \pm 1}, \ldots, x_{r}^{ \pm 1}\right]^{\mathfrak{S}_{r}}$ as soon as $r>2$ and $g>0$.

(b) The above proposition holds for the Hall algebras $\mathbf{U}_{K_{a}}^{>}$only, or alternatively when the curve $X$ is generic. For special curves the Frobenius eigenvalues might not all be distinct (for instance, some of them could be real); this corresponds to the merging of two irreducible components $Z_{r}^{(\alpha)}, Z_{r}^{\left(\alpha^{\prime}\right)}$.

1.13. In this last section, we define a twisted version $\dot{\mathbf{U}}_{X}^{>}$of the spherical Hall algebra $\mathbf{U}_{X}^{>}$of $X$. This twisted version is more relevant to the geometric Langlands program. The operation of tensoring with a line bundle is an exact autoequivalence of the category $\operatorname{Coh}(X)$ and it induces an automorphism of the Hall algebra $\mathbf{H}_{X}$. Since

$$
\mathcal{L} \otimes \mathbf{1}_{1, d}^{\mathbf{s s}}=\mathbf{1}_{d+\operatorname{deg}(\mathcal{L})}^{\mathrm{ss}}, \quad d \in \mathbb{Z},
$$


tensoring with a line bundle preserves the subalgebra $\mathbf{U}_{X}^{>}$. Let $\Omega \in P i c^{2(g-1)}(X)$ be the canonical bundle of $X$. We define a twisted multiplication in $\mathbf{U}_{X}^{>}$by the rule

$$
u_{r} \circ w_{s}=\left(u_{r} \otimes \Omega^{-s / 2}\right) \cdot\left(w_{s} \otimes \Omega^{r / 2}\right), \quad u_{r} \in \mathbf{U}_{X}^{>}[r], \quad w_{s} \in \mathbf{U}_{X}^{>}[s] .
$$

The new multiplication $\circ$ is associative. We denote by $\dot{\mathbf{U}}_{X}^{>}$the ensuing algebra. Let us rewrite the new multiplication in terms of the shuffle algebra $\mathbf{A}=\mathbf{A}_{g_{X}(z)}$. We have

$$
J_{r}\left(u_{r} \otimes \Omega^{n / 2}\right)=\left(x_{1} \cdots x_{r}\right)^{(g-1) n} J_{r}\left(u_{r}\right), \quad n \in \mathbb{Z}, \quad u_{r} \in \mathbf{U}_{X}^{>}[r] .
$$

Hence the twisted multiplication in $\mathbf{A}_{g_{X}(z)}$ is given by

$$
\begin{aligned}
& P\left(x_{1}, \ldots, x_{r}\right) \circ Q\left(x_{1}, \ldots, x_{s}\right)=\left(\left(x_{1} \cdots x_{r}\right)^{(1-g) s} P\left(x_{1}, \ldots, x_{r}\right)\right) \star\left(\left(x_{1} \cdots x_{s}\right)^{(g-1) r} Q\left(x_{1}, \ldots, x_{s}\right)\right) \\
& =\sum_{\sigma \in S h_{r, s}} \sigma\left\{\prod_{\substack{1 \leqslant i \leqslant r \\
r+1 \leqslant j \leqslant s}} g_{X}\left(x_{i} / x_{j}\right)\left(x_{1} \cdots x_{r}\right)^{(1-g) s}\left(x_{r+1} \cdots x_{r+s}\right)^{(g-1) r} P\left(x_{1}, \ldots, x_{r}\right) Q\left(x_{r+1}, \ldots, x_{r+s}\right)\right\} \\
& =\sum_{\sigma \in S h_{r, s}} \sigma\left\{\prod_{\substack{1 \leqslant i \leqslant r \\
r+1 \leqslant j \leqslant s}} g_{X}^{\prime}\left(x_{i} / x_{j}\right) P\left(x_{1}, \ldots, x_{r}\right) Q\left(x_{r+1}, \ldots, x_{r+s}\right)\right\}
\end{aligned}
$$

with $g_{X}^{\prime}(z)=z^{(1-g)} g(z)=\tilde{\zeta}_{X}\left(z^{-1}\right)$. In other terms, we have the following.

Corollary 1.17. The assignement $\mathbf{1}_{1, d}^{s s} \mapsto x_{1}^{d}, d \in \mathbb{Z}$, extends to an algebra isomorphism

$$
\dot{\Upsilon}_{X}: \dot{\mathbf{U}}_{X}^{>} \rightarrow \mathbf{A}_{\tilde{\zeta}_{X}\left(z^{-1}\right)}
$$

such that $\dot{\Upsilon}_{X}\left(u_{0} \bullet v\right)=\pi_{r}\left(u_{0}\right) \cdot \dot{\Upsilon}_{X}(v)$ for $u_{0} \in \mathbf{U}_{X}^{0}$ and $v \in \dot{\mathbf{U}}_{X}^{>}[r]$.

We also have identifications of integral and rational forms

$$
\dot{\mathbf{U}}_{R_{a}}^{>} \simeq \mathbf{A}_{R_{a}, \tilde{\zeta}\left(z^{-1}\right)}, \quad \dot{\mathbf{U}}_{K_{a}}^{>} \simeq \mathbf{A}_{K_{a}, \tilde{\zeta}\left(z^{-1}\right)} .
$$

Remark 1.18. (a) The twist (1.26) is classical, see e.g., [Ga]. It may be written in a slightly more canonical fashion as follows. Identify, for $r \geqslant 1$, the algebra $\mathbb{C}\left[x_{1}^{ \pm 1}, \ldots, x_{r}^{ \pm 1}\right]^{\mathfrak{S}_{r}}$ with $\mathbb{C}[T]^{W}$, where $T \subset G=G L_{r}$ is a maximal torus and $W=\mathfrak{S}_{r}$ is the Weyl group of $(G, T)$. This way we view the multiplication in $\mathbf{A}_{g(z)}$ as a collection of (induction) maps

$$
m_{L, G}: \mathbb{C}\left[T_{L}\right]^{W_{L}} \rightarrow \mathbb{C}\left[T_{G}\right]^{W_{G}},
$$

where $L$ is the Levi factor of some parabolic subgroup $P \subset G$ and $G$ runs among all $G L_{r}$. Then the twisted multiplication can be written as

$$
\dot{m}_{L, G}(f)=m_{L, G}\left(e^{2(1-g)\left(\rho_{G}-\rho_{L}\right)} f\right),
$$

where $\rho_{H}$ is half the sum of positive roots of a reductive group $H$.

(b) The main reason for considering $\dot{\mathbf{U}}_{X}^{>}$rather than $\mathbf{U}_{X}^{>}$is that the functional equation for Eisenstein series takes in $\dot{\mathbf{U}}_{X}^{>}$the following particularly nice form. Indeed, the series

$$
\dot{E}\left(z_{1}, \ldots, z_{r}\right)=E_{1}\left(z_{1}\right) \circ \cdots \circ E_{1}\left(z_{r}\right), \quad E_{1}(z)=\sum_{d \in \mathbb{Z}} \mathbf{1}_{1, d} z^{d},
$$

viewed as a rational function in $z_{1}, \ldots, z_{r}$, is symmetric. 


\section{K-Theoretic Hall algebras}

2.1. Let $G$ be a complex linear algebraic group. By a variety we'll always mean a quasi-projective complex variety. We call $G$-variety a variety $X$ with a rational action of $G$. Let $K^{G}(X)$ be the complexified Grothendieck group of the Abelian category of the $G$-equivariant coherent sheaves over $X$. Let $P \subset G$ a parabolic subgroup and $H \subset P$ a Levi subgroup. Fix a $H$-variety $Y$. The group $P$ acts on $Y$ through the obvious group homomorphism $P \rightarrow H$. The induced $G$-variety is

$$
X=G \times{ }_{P} Y .
$$

Now assume that $Y$ is smooth. Given a smooth subscheme $O \subset Y$ let $T_{O}^{*} Y \subset T^{*} Y$ be the conormal bundle to $O$. It is well-known that the induced $H$-action on $T^{*} Y$ is Hamiltonian and that the zero set of the moment map is the closed $H$-subvariety

$$
T_{H}^{*} Y=\bigcup_{O} T_{O}^{*} Y \subset T^{*} Y
$$

where $O$ runs over the set of $H$-orbits. The following lemma is left to the reader.

Lemma 2.1. We have $T^{*} X=T_{P}^{*}(G \times Y) / P$ and $T_{G}^{*} X=G \times_{P} T_{H}^{*} Y$. The induction yields a canonical isomorphism $K^{H}\left(T_{H}^{*} Y\right)=K^{G}\left(T_{G}^{*} X\right)$.

We'll call fibration a smooth morphism which is locally trivial in the Zariski topology. Let $X^{\prime}$ be a smooth $G$-variety and $V$ be a smooth $H$-variety. Assume that we are given $H$-equivariant homomorphisms

$$
Y \stackrel{p}{\longleftarrow} V \stackrel{q}{\longrightarrow} X^{\prime}
$$

such that $p$ is a fibration and $q$ is a closed embedding. Set

$$
W=G \times{ }_{P} V
$$

and consider the following maps

$$
\begin{gathered}
X \stackrel{f}{\longleftarrow} W \stackrel{g}{\longrightarrow} X^{\prime} \\
f:(g, v) \bmod P \mapsto(g, p(v)) \bmod P, \quad g:(g, v) \bmod P \mapsto g q(v) .
\end{gathered}
$$

The following properties are immediate.

Lemma 2.2. The map $f$ is a G-equivariant fibration, the map $g$ is a G-equivariant proper morphism, and the map $(f, g)$ is a closed embedding $W \subset X \times X^{\prime}$. The varieties $V, W, X, X^{\prime}$ are smooth.

We'll identify $W$ with its image in $X \times X^{\prime}$. Let $Z=T_{W}^{*}\left(X \times X^{\prime}\right)$ be the conormal bundle. It is again a smooth $G$-variety. The obvious projections yield $G$-equivariant maps

$$
T^{*} X \stackrel{\phi}{\longleftarrow} Z \stackrel{\psi}{\longrightarrow} T^{*} X^{\prime} .
$$

Consider the $G$-variety

$$
Z_{G}=Z \cap\left(T_{G}^{*} X \times T_{G}^{*} X^{\prime}\right) .
$$

Recall that a morphism of varieties $S \rightarrow T$ is called regular if it is the composition of a regular immersion $S \subset S^{\prime}$, i.e., an immersion which is locally defined by a regular sequence, and of a smooth map $S^{\prime} \rightarrow T$. Note that a regular map has a finite tor-dimension and that a morphism $S \rightarrow T$ is regular whenever $S, T$ are smooth. The following is immediate.

Lemma 2.3. (a) The map $\psi$ is proper and regular, the map $\phi$ is regular.

(b) We have $\phi^{-1}\left(T_{G}^{*} X\right)=Z_{G}$ and $\psi\left(Z_{G}\right) \subset T_{G}^{*} X^{\prime}$. 
We'll abbreviate $\phi_{G}=\left.\phi\right|_{Z_{G}}$ and $\psi_{G}=\left.\psi\right|_{Z_{G}}$. We have the following diagram

$$
T_{G}^{*} X \stackrel{\phi_{G}}{\longleftarrow} Z_{G} \stackrel{\psi_{G}}{\longrightarrow} T_{G}^{*} X^{\prime} .
$$

Recall that for any $G$-variety $M$ and any closed $G$-stable subvariety $N \subset M$ the direct image by the obvious inclusion $N \rightarrow M$ identifies $K^{G}(N)$ with the complexified Grothendieck group $K^{G}(M$ on $N)$ of the category of $G$-equivariant coherent sheaves over $M$ supported on $N$. Since the map $\psi$ is a proper morphism the derived direct image yields maps

$$
\begin{gathered}
R \psi_{*}: K^{G}(Z) \rightarrow K^{G}\left(T^{*} X^{\prime}\right), \\
R \psi_{*}: K^{G}\left(Z_{G}\right)=K^{G}\left(Z \text { on } Z_{G}\right) \rightarrow K^{G}\left(T^{*} X^{\prime} \text { on } T_{G}^{*} X^{\prime}\right)=K^{G}\left(T_{G}^{*} X^{\prime}\right) .
\end{gathered}
$$

Since the map $\phi$ has a finite tor-dimension the derived pull-back yields maps

$$
\begin{gathered}
L \phi^{*}: K^{G}\left(T^{*} X\right) \rightarrow K^{G}(Z), \\
L \phi^{*}: K^{G}\left(T_{G}^{*} X\right)=K^{G}\left(T^{*} X \text { on } T_{G}^{*} X\right) \rightarrow K^{G}\left(Z \text { on } Z_{G}\right)=K^{G}\left(Z_{G}\right) .
\end{gathered}
$$

By definition $L \phi^{*}$ is the composition of the pull-back by the projection $T^{*} X \times T^{*} X^{\prime} \rightarrow T^{*} X$ and the derived pull-back by the regular immersion $Z \subset T^{*} X \times T^{*} X^{\prime}$. Composing $R \psi_{*}$ and $L \phi^{*}$ we get a map

$$
R \psi_{*} \circ L \phi^{*}: K^{G}\left(T_{G}^{*} X\right) \rightarrow K^{G}\left(T_{G}^{*} X^{\prime}\right) .
$$

By Lemma 2.1 the induction yields also an isomorphism

$$
K^{H}\left(T_{H}^{*} Y\right)=K^{G}\left(T_{G}^{*} X\right) .
$$

Composing it by $R \psi_{*} \circ L \phi^{*}$ we obtain a map

$$
K^{H}\left(T_{H}^{*} Y\right) \rightarrow K^{G}\left(T_{G}^{*} X^{\prime}\right)
$$

2.2. We'll apply the general construction recalled above to the following particular case. First, let us fix some notation. Let $E$ be a finite dimensional $\mathbb{C}$-vector space. We'll abbreviate

$$
G_{E}=G L(E), \quad \mathfrak{g}_{E}=\operatorname{End}(E) .
$$

We set

$$
\begin{gathered}
C_{E}=\left\{(a, b) \in\left(\mathfrak{g}_{E}\right)^{g} \times\left(\mathfrak{g}_{E}\right)^{g} ; \sum_{r}\left[a_{r}, b_{r}\right]=0\right\}, \\
a=\left(a_{1}, a_{2}, \ldots a_{g}\right), \quad b=\left(b_{1}, b_{2}, \ldots b_{g}\right) .
\end{gathered}
$$

If no confusion is possible we'll write $C=C_{E}, G=G_{E}$ and $\mathfrak{g}=\mathfrak{g}_{E}$. Let also

$$
\begin{aligned}
T_{s} & =\left\{(h, e, f) \in\left(\mathbb{C}^{\times}\right)^{2 g+1} ; e_{r} f_{r}=h, \forall r\right\}, \\
e & =\left(e_{1}, e_{2}, \ldots e_{g}\right), \quad f=\left(f_{1}, f_{2}, \ldots f_{g}\right) .
\end{aligned}
$$

Thus $T_{s}$ is a $g+1$-dimensional torus which acts on $C$ in the following way

$$
(h, e, f) \cdot(a, b)=\left(e_{1} a_{1}, e_{2} a_{2}, \ldots, f_{1} b_{1}, \ldots f_{g} b_{g}\right) .
$$

We may abbreviate $(e \cdot a, f \cdot b)$ for the right hand side. We also equip $C$ with the diagonal $G$-action such that $G$ acts on $\mathfrak{g}$ by the adjoint action. The $T_{s}$-action and the $G$-action on $C$ commute, yielding an action of the group $T_{s} \times G$. Let $R_{s}$ be the complexified representation ring of $T_{s}$. We have

$$
\begin{gathered}
R_{s}=\mathbb{C}\left[p^{ \pm 1}, x_{1}^{ \pm 1}, y_{1}^{ \pm 1}, \ldots, x_{g}^{ \pm 1}, y_{g}^{ \pm 1}\right] /\left(p-x_{r} y_{r}\right), \\
p(h, e, f)=h, \quad x_{r}(h, e, f)=e_{r}, \quad y_{r}(h, e, f)=f_{r} .
\end{gathered}
$$


2.3. Next we fix a flag

$$
0 \rightarrow E_{1} \rightarrow E \rightarrow E_{2} \rightarrow 0 .
$$

Set $H=G_{E_{1}} \times G_{E_{2}}$ and $P=\left\{g \in G ; g\left(E_{1}\right)=E_{1}\right\}$. Let $\mathfrak{h}$ and $\mathfrak{p}$ be the corresponding Lie algebras. Put $Y=\mathfrak{h}^{g}, V=\mathfrak{p}^{g}$, and $X^{\prime}=\mathfrak{g}^{g}$. The $G$-action on $X^{\prime}$ and the $H$-action on $Y$ are the adjoint ones. Put also

$$
C_{\mathfrak{g}}=C_{E}, \quad C_{\mathfrak{h}}=C_{E_{1}} \times C_{E_{2}}, \quad C_{\mathfrak{p}}=\left(\mathfrak{p}^{g} \times \mathfrak{p}^{g}\right) \cap C_{E} .
$$

Let $a \mapsto p(a)=a_{\mathfrak{h}}$ denote the canonical maps $\mathfrak{p} \rightarrow \mathfrak{h}$ and $\mathfrak{p}^{g} \rightarrow \mathfrak{h}^{g}$. We apply the general construction in Section 2.2 to the diagram

$$
Y \stackrel{p}{\longleftarrow} V \stackrel{q}{\longrightarrow} X^{\prime}
$$

where $q$ is the obvious inclusion. We have the following lemma. Here the $P$-actions on $\mathfrak{p}^{g} \times \mathfrak{p}^{g}$ and on $\mathfrak{p} \times \mathfrak{h}^{g} \times \mathfrak{h}^{g}$ are the obvious ones, the $G$-action on $T^{*} X^{\prime}$ is as in Section 2.2, and the $G$-action on $T^{*} X, Z$ is by left multiplication on $G$. Further we identify $\mathfrak{g}^{*}=\mathfrak{g}$ and $\mathfrak{h}^{*}=\mathfrak{h}$ via the trace.

Lemma 2.4. (a) There are isomorphisms of $G$-varieties

$$
\begin{gathered}
T^{*} X^{\prime}=\mathfrak{g}^{g} \times \mathfrak{g}^{g}, \quad Z=G \times{ }_{P}\left(\mathfrak{p}^{g} \times \mathfrak{p}^{g}\right), \\
T^{*} X=G \times{ }_{P}\left\{(d, a, b) \in \mathfrak{p} \times \mathfrak{h}^{g} \times \mathfrak{h}^{g} ; d_{\mathfrak{h}}=\sum_{r}\left[a_{r}, b_{r}\right]\right\} .
\end{gathered}
$$

For each $(a, b) \in \mathfrak{p}^{g} \times \mathfrak{p}^{g}$ we have

$$
\begin{gathered}
\phi((g, a, b) \bmod P)=\left(g, \sum_{r}\left[a_{r}, b_{r}\right], a_{\mathfrak{h}}, b_{\mathfrak{h}}\right) \bmod P \\
\psi((g, a, b) \bmod P)=\left(g a g^{-1}, g b g^{-1}\right) .
\end{gathered}
$$

(b) There are isomorphisms of $G$-varieties

$$
T_{G}^{*} X^{\prime}=C_{\mathfrak{g}}, \quad Z_{G}=G \times{ }_{P} C_{\mathfrak{p}}, \quad T_{G}^{*} X=G \times{ }_{P} C_{\mathfrak{h}} .
$$

The maps $\phi_{G}, \psi_{G}$ are the obvious ones.

Proof. The linear map $\delta: \mathfrak{p} \rightarrow \mathfrak{h} \times \mathfrak{g}, a \mapsto\left(a_{\mathfrak{h}}, a\right)$ is $P$-equivariant. We'll identify $\mathfrak{p}$ and $\delta \mathfrak{p}$ whenever needed. By Lemma 2.1 we have

$$
\begin{aligned}
T^{*}\left(X \times X^{\prime}\right) & =T_{P}^{*}\left(G \times \mathfrak{h}^{g} \times \mathfrak{g}^{g}\right) / P \\
& =G \times{ }_{P}\left\{(a, f) \in\left(\mathfrak{h}^{g} \times \mathfrak{g}^{g}\right) \times\left(\mathfrak{g} \times \mathfrak{h}^{g} \times \mathfrak{g}^{g}\right)^{*} ; f(-b,[\delta b, a])=0, \forall b \in \mathfrak{p}\right\}, \\
T^{*} X & =T_{P}^{*}\left(G \times \mathfrak{h}^{g}\right) / P \\
& =G \times{ }_{P}\left\{(a, f) \in \mathfrak{h}^{g} \times\left(\mathfrak{g} \times \mathfrak{h}^{g}\right)^{*} ; f\left(-b,\left[b_{\mathfrak{h}}, a\right]\right)=0, \forall b \in \mathfrak{p}\right\}, \\
T^{*} X^{\prime} & =\mathfrak{g}^{g} \times\left(\mathfrak{g}^{*}\right)^{g}=\mathfrak{g}^{g} \times \mathfrak{g}^{g} .
\end{aligned}
$$

Fix $f=(\lambda, \alpha) \in \mathfrak{g}^{*} \times\left(\mathfrak{h}^{g}\right)^{*}$. For each $a \in \mathfrak{h}^{g}$ we have

$$
f\left(-b,\left[b_{\mathfrak{h}}, a\right]\right)=0, \forall b \in \mathfrak{p} \Longleftrightarrow \lambda(b)=\alpha\left(\left[b_{\mathfrak{h}}, a\right]\right), \forall b \in \mathfrak{p} .
$$

Let $\mathfrak{p}_{\text {nil }} \subset \mathfrak{p}$ be the nilpotent radical. Thus the left hand side is satisfied iff we have

$$
\lambda\left(\mathfrak{p}_{n i l}\right)=0,\left.\quad \lambda\right|_{\mathfrak{h}}=\sum_{r} \operatorname{ad}^{*}\left(a_{r}\right)\left(\alpha_{r}\right),
$$

where $\mathrm{ad}^{*}$ is the coadjoint action. Under the canonical isomorphism $\mathfrak{g}^{*} \rightarrow \mathfrak{g}$ this yields the formula for $T^{*} X$ in the lemma. Next we have $W=G \times{ }_{P} \mathfrak{p}^{g}$ and $X \times X^{\prime}=G \times{ }_{P}\left(\mathfrak{h}^{g} \times \mathfrak{g}^{g}\right)$. Further the inclusion $W \subset X \times X^{\prime}$ is given by the map $\delta$. We have also

$$
\begin{aligned}
T^{*} W & =T_{P}^{*}\left(G \times \mathfrak{p}^{g}\right) / P \\
& =G \times{ }_{P}\left\{(a, f) \in \mathfrak{p}^{g} \times\left(\mathfrak{g} \times \mathfrak{p}^{g}\right)^{*} ; f(-b,[b, a])=0, \forall b \in \mathfrak{p}\right\} .
\end{aligned}
$$


Let $\mathfrak{p}^{\perp} \subset(\mathfrak{h} \times \mathfrak{g})^{*}$ be the orthogonal of $\delta \mathfrak{p}$. We get the following isomorphisms

$$
\begin{gathered}
\left.T^{*}\left(X \times X^{\prime}\right)\right|_{W}=G \times{ }_{P}\left\{(a, f) \in \mathfrak{p}^{g} \times\left(\mathfrak{g} \times \mathfrak{h}^{g} \times \mathfrak{g}^{g}\right)^{*} ; f(-b, \delta[b, a])=0, \forall b \in \mathfrak{p}\right\}, \\
Z=T_{W}^{*}\left(X \times X^{\prime}\right)=G \times{ }_{P}\left(\mathfrak{p}^{g} \times\left(\mathfrak{p}^{\perp}\right)^{g}\right) .
\end{gathered}
$$

The canonical isomorphism $(\mathfrak{h} \times \mathfrak{g})^{*} \rightarrow \mathfrak{h} \times \mathfrak{g}$ identifies $\mathfrak{p}^{\perp}$ with

$$
\mathfrak{p}^{\prime}=\left\{\left(-a_{\mathfrak{h}}, a\right) \in \mathfrak{h} \times \mathfrak{g} ; a \in \mathfrak{p}\right\} .
$$

Note that $\mathfrak{p}^{\prime}=\mathfrak{p}$ as a $P$-module. This yields an isomorphism

$$
Z=G \times{ }_{P}\left(\mathfrak{p}^{g} \times \mathfrak{p}^{g}\right) .
$$

The inclusion $Z \subset T^{*}\left(X \times X^{\prime}\right)$ is given by the map $\delta: \mathfrak{p}^{g} \rightarrow \mathfrak{h}^{g} \times \mathfrak{g}^{g}$ and by the map

$$
\mathfrak{p}^{g}=\left(\mathfrak{p}^{\prime}\right)^{g}=\{0\} \times\left(\mathfrak{p}^{\perp}\right)^{g} \subset\{0\} \times\left(\mathfrak{h}^{g} \times \mathfrak{g}^{g}\right)^{*} \subset\left(\mathfrak{g} \times \mathfrak{h}^{g} \times \mathfrak{g}^{g}\right)^{*} .
$$

The maps $\phi, \psi$ are composed of the inclusion $Z \subset T^{*}\left(X \times X^{\prime}\right)$ and the projections to $T^{*} X$, $T^{*} X^{\prime}$. Fix $a, b \in \mathfrak{p}^{g}$. Consider the element $\xi \in Z$ equal to $(g, a, b)$ modulo $P$. We may identify $a$ with $\delta a=\left(a_{\mathfrak{h}}, a\right)$, which is an element of $\mathfrak{h}^{g} \times \mathfrak{g}^{g}$, and $b$ with the element $\left(-b_{\mathfrak{h}}, b\right) \in\left(\mathfrak{p}^{\prime}\right)^{g}$, which can be regarded as an element in

$$
\left(\mathfrak{p}^{\perp}\right)^{g} \subset\left(\mathfrak{h}^{g} \times \mathfrak{g}^{g}\right)^{*}=\{0\} \times\left(\mathfrak{h}^{g} \times \mathfrak{g}^{g}\right)^{*} \subset\left(\mathfrak{g} \times \mathfrak{h}^{g} \times \mathfrak{g}^{g}\right)^{*} .
$$

So $\xi$ can be viewed as an element in $T^{*}\left(X \times X^{\prime}\right)$, and we have

$$
\psi(\xi)=\left(g a g^{-1}, g b g^{-1}\right), \quad \phi(\xi)=\left(g,-\left[a_{\mathfrak{h}}, b\right], a_{\mathfrak{h}},-b_{\mathfrak{h}}\right) \bmod P .
$$

This yields the formulas for $\phi$ and $\psi$ in the lemma. The claim $(b)$ is left to the reader.

Next, we set $\mathbf{C}_{E}=K^{T_{s} \times G}\left(C_{\mathfrak{g}}\right)$. A vector space isomorphism $E \simeq E^{\prime}$ yields an $R_{s}$-module isomorphism $\mathbf{C}_{E} \simeq \mathbf{C}_{E^{\prime}}$. Let $\mathbf{C}=\lim _{\rightarrow} \mathbf{C}_{E}$, where the colimit runs over the groupoid formed by all finite dimensional vector spaces with their isomorphisms. There is a $T_{s} \times G$-action on $T^{*} X$, $T^{*} X^{\prime}$ such that $G$ acts as above and $T$ acts by

$$
\begin{gathered}
(h, e, f) \cdot(g, d, a, b) \bmod P=(g, h \cdot d, e \cdot a, f \cdot b) \bmod P, \\
(h, e, f) \cdot(g, a, b) \bmod P=(g, e \cdot a, f \cdot b) \bmod P .
\end{gathered}
$$

Here the symbol $h \cdot d$ is simply the multiplication of $d$ by the scalar $h$. We define as in (2.2) a $R_{s}$-linear homomorphism

$$
K^{T_{s} \times H}\left(C_{\mathfrak{h}}\right) \rightarrow K^{T_{s} \times G}\left(C_{\mathfrak{g}}\right) .
$$

By the Kunneth formula [CG, Chap. 5.6.], it can be viewed as a map

$$
\mathbf{C}_{E_{1}} \otimes_{R_{s}} \mathbf{C}_{E_{2}} \rightarrow \mathbf{C}_{E} .
$$

The following is proved as in [SV2, Proposition 7.5].

Proposition 2.5. The map (2.5) equips $\mathbf{C}$ with the structure of a $R_{s}$-algebra with 1.

2.4. We'll call the $R_{s}$-algebra $\mathbf{C}$ the $K$-theoretic Hall algebra. It is naturally $\mathbb{N}$-graded, with the piece of degree $n$ equal to the colimit over the groupoid formed by all $n$-dimensional vector spaces with their isomorphisms $\mathbf{C}_{n}=\lim _{\rightarrow E} \mathbf{C}_{E}$. The spherical subalgebra of $\mathbf{C}$ is the $R_{s}$-subalgebra $\mathbf{C}^{\prime} \subset \mathbf{C}$ generated by $\mathbf{C}_{1}$. We'll abbreviate

$$
\mathbf{C}_{n}^{\prime}=\mathbf{C}_{n} \cap \mathbf{C}^{\prime}, \quad \mathbf{C}_{K}^{\prime}=\mathbf{C}^{\prime} \otimes_{R_{s}} K_{s}, \quad \mathbf{C}_{K}=\mathbf{C} \otimes_{R_{s}} K_{s},
$$

where $K_{s}$ is the fraction field of $R_{s}$. Write also $R_{G}$ for the complexified representation ring of $T_{s} \times G$ and $K_{G}$ for its fraction field. For each $E$ as above the direct image by the obvious inclusion $C_{\mathfrak{g}} \subset \mathfrak{g}^{g} \times \mathfrak{g}^{g}$ yields a $R_{G^{-}}$-module homomorphism

$$
\mathbf{C}_{n} \rightarrow K^{T_{s} \times G}\left(\mathfrak{g}^{g} \times \mathfrak{g}^{g}\right) .
$$


We conjecture that (2.6) is an injective map. This conjecture is equivalent to the following one. Set $n=\operatorname{dim} E$ and $G=G L_{n}$.

Conjecture 2.6. The $R_{G L_{n}}$-module $\mathbf{C}_{n}$ is torsion-free.

The kernel of (2.6) is the torsion $R_{G L_{n}}$-submodule of $\mathbf{C}_{n}$. Let $\overline{\mathbf{C}}_{n}$ be the image of $\mathbf{C}_{n}^{\prime}$ by (2.6). We set

$$
\overline{\mathbf{C}}=\bigoplus_{n \geqslant 0} \overline{\mathbf{C}}_{n}
$$

Proposition 2.7. The map (2.6) yields a surjective $R$-algebra homomorphism $\mathbf{C}^{\prime} \rightarrow \overline{\mathbf{C}}$.

Proof. For any finite dimensional vector space $E$ let $\tilde{\mathbf{C}}_{E}$ be the quotient of $\mathbf{C}_{E}$ by is torsion $R_{G_{E}}$-submodule. Given $E_{1}, E_{2}, E$ as in Section 2.3, we must check that the map (2.5) fits into a commutative square

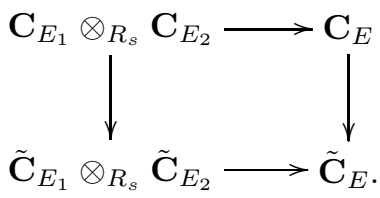

The upper arrow is identified with the map $K^{T_{s} \times H}\left(C_{\mathfrak{h}}\right) \rightarrow K^{T_{s} \times G}\left(C_{\mathfrak{g}}\right)$ which comes from (2.1). Further $\tilde{\mathbf{C}}_{E_{1}} \otimes_{R_{s}} \tilde{\mathbf{C}}_{E_{2}}$ and $\tilde{\mathbf{C}}_{E}$ are identified with the images by the obvious maps

$$
K^{T_{s} \times G}\left(T_{G}^{*} X\right) \rightarrow K^{T_{s} \times G}\left(T^{*} X\right), \quad K^{T_{s} \times G}\left(T_{G}^{*} X^{\prime}\right) \rightarrow K^{T_{s} \times G}\left(T^{*} X^{\prime}\right)
$$

respectively. So the proposition follows from the commutativity of the square

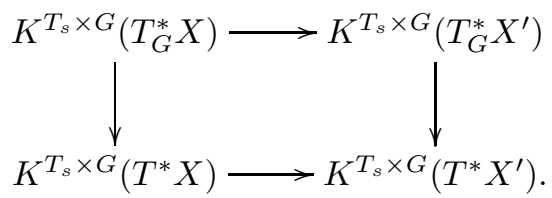

2.5. Fix a $n$-dimensional vector space $E$. Let $H \subset G$ be the torus consisting of the diagonal matrices. The inverse image by the obvious inclusion $i:\{0\} \rightarrow \mathfrak{g}^{g} \times \mathfrak{g}^{g}$ yields a map

$$
L i^{*}: K^{T_{s} \times G}\left(\mathfrak{g}^{g} \times \mathfrak{g}^{g}\right) \rightarrow R_{G} .
$$

Composing it with (2.6) we get a $R_{G}$-linear map

$$
\gamma_{G}: \mathbf{C}_{n} \rightarrow R_{G} .
$$

In the same way we have a $R_{H}$-linear map

$$
\gamma_{H}=\left(\gamma_{\mathbb{C}^{\times}}\right)^{\otimes n}:\left(\mathbf{C}_{1}\right)^{\otimes n} \rightarrow R_{H}
$$

(the tensor power over $R_{s}$ ). Write

$$
R_{\mathbb{C}^{\times}}=R_{s}\left[z^{ \pm 1}\right], \quad R_{H}=\left(R_{\mathbb{C}^{\times}}\right)^{\otimes n}=R_{s}\left[z_{1}^{ \pm 1}, z_{2}^{ \pm 1}, \ldots, z_{n}^{ \pm 1}\right], \quad R_{G}=R_{s}\left[z_{1}^{ \pm 1}, z_{2}^{ \pm 1}, \ldots, z_{n}^{ \pm 1}\right]^{\mathfrak{S}_{n}}
$$

Note that we have

$$
K_{\mathbb{C}^{\times}}=K_{s}(z), \quad K_{H}=K_{s}\left(z_{1}, z_{2}, \ldots, z_{n}\right), \quad K_{G}=K_{s}\left(z_{1}, z_{2}, \ldots, z_{n}\right)^{\mathfrak{S}_{n}} .
$$

Recall the standard symmetrization operator $\operatorname{Sym}_{n}: K_{H} \rightarrow K_{G}$. 
Proposition 2.8. We have the following commutative diagram

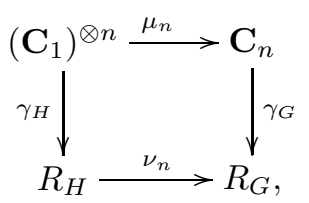

where the upper map is the multiplication in $\mathbf{C}$ and the map $\nu_{n}$ is given by

$$
\nu_{n}\left(P\left(z_{1}, \ldots, z_{n}\right)\right)=\operatorname{Sym}_{n}\left(k\left(z_{1}, z_{2}, \ldots z_{n}\right) P\left(z_{1}, z_{2}, \ldots z_{n}\right)\right),
$$

where

$$
\begin{gathered}
k\left(z_{1}, \ldots, z_{n}\right)=\prod_{i<j} k\left(z_{i} / z_{j}\right), \\
k(z)=(1-z)^{-1}\left(1-p^{-1} z^{-1}\right) \prod_{r}\left(1-x_{r}^{-1} z\right)\left(1-y_{r}^{-1} z\right) .
\end{gathered}
$$

Proof. Fix a monomial $\theta_{m}=z_{1}^{m_{1}} z_{2}^{m_{2}} \ldots z_{n}^{m_{n}}$ with $m=\left(m_{1}, m_{2}, \ldots m_{n}\right) \in \mathbb{Z}^{n}$. We'll regard $\theta_{m}$ as an element of $R_{H}$. Since $C_{\mathfrak{h}}$ is a vector space and $T_{s} \times H$ is a torus, the $R_{s}$-module $K^{T_{s} \times H}\left(C_{\mathfrak{h}}\right)$ is spanned by the classes of the $T_{s} \times H$-equivariant line bundles $\mathcal{O}_{C_{\mathfrak{h}}}\langle m\rangle$. Here the symbol $\langle m\rangle$ means the tensor product of $\mathcal{O}_{C_{\mathfrak{h}}}$, with the trivial action, by the character $\theta_{m}$. Note that

$$
\gamma_{H}\left(\mathcal{O}_{C_{\mathfrak{h}}}\langle m\rangle\right)=\theta_{m}
$$

Let $B \subset G$ be the Borel subgroup consisting of upper triangular matrices. Let $\mathfrak{b}$ be the Lie algebra of $B$ and $\mathfrak{n}$ be the nilpotent radical of $\mathfrak{b}$. Recall that we have

$$
T_{G}^{*} X=G \times{ }_{B} C_{\mathfrak{h}}, \quad T^{*} X=G \times{ }_{B}\left(\mathfrak{n} \times C_{\mathfrak{h}}\right), \quad C_{\mathfrak{h}}=\mathfrak{h}^{g} \times \mathfrak{h}^{g} .
$$

Let $\Gamma$ denote the induction of equivariant sheaves from $T_{s} \times B$ to $T_{s} \times G$. Consider the $T_{s} \times G-$ equivariant line bundle over $T_{G}^{*} X$

$$
\mathcal{O}_{T_{G}^{*} X}(m)=\Gamma\left(\mathcal{O}_{C_{\mathfrak{h}}}\langle m\rangle\right) .
$$

For a future use, let us consider the following commutative diagram

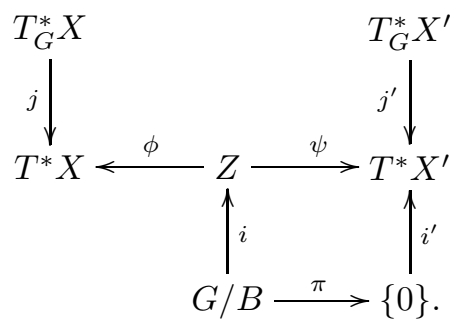

Here $j, j^{\prime}, i, i^{\prime}$ are the obvious inclusions. By definition of the multiplication in (2.1) we have

$$
\nu_{n}\left(\theta_{m}\right)=\gamma_{G}\left(\mathcal{E}_{m}\right)=L\left(i^{\prime}\right)^{*} j_{*}^{\prime}\left(\mathcal{E}_{m}\right)
$$

where $\mathcal{E}_{m}$ denotes an element of $K^{T_{s} \times G}\left(T_{G}^{*} X^{\prime}\right)$ whose image by $j_{*}^{\prime}$ is equal to

$$
R \psi_{*} L \phi^{*} j_{*}\left(\mathcal{O}_{T_{G}^{*} X}(m)\right) .
$$

Therefore we have the following formula

$$
\nu_{n}\left(\theta_{m}\right)=L\left(i^{\prime}\right)^{*} R \psi_{*} L \phi^{*} j_{*}\left(\mathcal{O}_{T_{G}^{*} X}(m)\right) .
$$

Now, we compute the right hand side of (2.10). Recall that $Z=G \times{ }_{B}\left(\mathfrak{b}^{g} \times \mathfrak{b}^{g}\right)$ and that $T^{*} X^{\prime}=\mathfrak{g}^{g} \times \mathfrak{g}^{g}$. For any finite dimensional $T_{s} \times B$-module $V$ we'll abbreviate

$$
\Lambda_{-1}(V)=\sum_{r \geqslant 0}(-1)^{r} \Lambda^{r}(V) \in R_{B} .
$$


We have

$$
j_{*}\left(\mathcal{O}_{T_{G}^{*} X}(m)\right)=\Gamma\left(\theta_{m} \otimes \Lambda_{-1}(p \mathfrak{n})^{*} \otimes \mathcal{O}_{\mathfrak{n} \times C_{\mathfrak{h}}}\right) .
$$

Therefore we have also

$$
L \phi^{*} j_{*}\left(\mathcal{O}_{T_{G}^{*} X}(m)\right)=\Gamma\left(\theta_{m} \otimes \Lambda_{-1}(p \mathfrak{n})^{*} \otimes \mathcal{O}_{\mathfrak{b}^{g} \times \mathfrak{b} g}\right) .
$$

Under tensoring by $K_{G}$ the maps $i_{*}, L i^{*}$ become invertible by the Thomason localization theorem. We'll abbreviate $x \mathfrak{b}=\sum_{r} x_{r} \mathfrak{b}$ and $y \mathfrak{b}=\sum_{r} y_{r} \mathfrak{b}$. We have

$$
\begin{aligned}
\nu_{n}\left(\theta_{m}\right) & =L\left(i^{\prime}\right)^{*} R \psi_{*} i_{*} \Gamma\left(\theta_{m} \otimes \Lambda_{-1}(p \mathfrak{n}-x \mathfrak{b}-y \mathfrak{b})^{*}\right) \\
& =L\left(i^{\prime}\right)^{*} i_{*}^{\prime} R \pi_{*} \Gamma\left(\theta_{m} \otimes \Lambda_{-1}(p \mathfrak{n}-x \mathfrak{b}-y \mathfrak{b})^{*}\right), \\
& =\Lambda_{-1}(x \mathfrak{g}+y \mathfrak{g})^{*} \otimes R \pi_{*} \Gamma\left(\theta_{m} \otimes \Lambda_{-1}(p \mathfrak{n}-x \mathfrak{b}-y \mathfrak{b})^{*}\right), \\
& =R \pi_{*} \Gamma\left(\theta_{m} \otimes \Lambda_{-1}\left(p \mathfrak{n}+x \mathfrak{n}^{*}+y \mathfrak{n}^{*}\right)^{*}\right) .
\end{aligned}
$$

Thus the integration over the fixed points subset $(G / B)^{H}$ of $G / B$ yields the desired formula

$$
\nu_{n}\left(\theta_{m}\right)=\operatorname{Sym}_{n}\left(k\left(z_{1}, z_{2}, \ldots z_{n}\right) \theta_{m}\right)
$$

where

$$
\begin{gathered}
k\left(z_{1}, z_{2}, \ldots z_{n}\right)=\prod_{i<j} k\left(z_{i} / z_{j}\right), \\
k(z)=(1-z)^{-1}\left(1-p^{-1} z^{-1}\right) \prod_{r}\left(1-x_{r}^{-1} z\right)\left(1-y_{r}^{-1} z\right) .
\end{gathered}
$$

2.6. Let $k(z)$ be given by (2.8). Then we have

$$
\mathbf{A}_{k(z)} \subset \bigoplus_{n} R_{s}\left[z_{1}^{ \pm 1}, \ldots, z_{n}^{ \pm 1}\right]^{\mathfrak{S}_{n}}
$$

Comparing formula (2.7) with the definition of shuffle algebras given in Section 1.9 yields the following corollary to Proposition 2.8.

Theorem 2.9. The maps $\overline{\mathbf{C}}_{n} \rightarrow K_{G L_{n}}, x \mapsto L i^{*}(x)$ give rise to a graded algebra isomorphism

$$
\Phi: \overline{\mathbf{C}} \stackrel{\sim}{\rightarrow} \mathbf{A}_{k(z)}
$$

such that $\Phi(\theta x)=\theta \cdot \Phi(x)$ for $x \in \overline{\mathbf{C}}_{n}$ and $\theta \in R_{G L_{n}}$.

\section{The ISOMORPHISM}

In this section we state our main result (whose proof is now obvious) relating the spherical $K$-theoretic Hall algebra $\overline{\mathbf{C}}$ and the (twisted) spherical Hall algebra $\dot{\mathbf{U}}_{X}^{>}$of a smooth projective curve $X$. As an application, we compute the images, under our correspondence, of the skyscraper sheaf $\mathcal{O}_{\text {triv }}$ at the trivial local system of rank $r$ over $X$ and of the constant function $\mathbf{1}_{r}^{\text {vec }}$ over Bun $_{G L_{r}} X$. 
3.1. Recall that we have fixed an identification

$$
\mathbf{C}_{1}=K^{T_{s} \times \mathbb{C}^{\times}}\left(\mathbb{C}^{2 g}\right) \stackrel{\sim}{\rightarrow} R_{s}\left[z_{1}^{ \pm 1}\right], \quad z^{d}\left[\mathcal{O}_{\mathbb{C}^{2 g}}\right] \mapsto z_{1}^{d}, \quad d \in \mathbb{Z} .
$$

We identify the torus $T_{a}$ arising on the Hall algebra side, see (1.23), with the torus $T_{s}$ arising on the K-theory side, see (2.3), via the map

$$
\eta_{i} \mapsto e_{i}^{-1}, \quad \bar{\eta}_{i} \mapsto f_{i}^{-1}, \quad i=1, \ldots, g .
$$

This induces a ring isomorphism

$$
R_{a} \simeq R_{s}, \quad \alpha_{i} \mapsto x_{i}^{-1}, \quad \bar{\alpha}_{i} \mapsto y_{i}^{-1}, \quad q \mapsto p^{-1} .
$$

From now on we will simply write $R$ for rings $R_{a}, R_{s}$ and $K$ for the fraction field of $R$. Observe that under the identification (3.1) we have $\tilde{\zeta}(z)=k(z)$, see Sections 1.9 and 2.5. Note also that we have, in $K^{T_{s} \times \mathbb{C}^{\times}}\left(\mathbb{C}^{2 g}\right)$,

$$
\left[\mathcal{O}_{\{0\}}\right]=\prod_{l=1}^{g}\left(1-x_{l}\right)\left(1-y_{l}\right)\left[\mathcal{O}_{\mathbb{C}^{2 g}}\right]=q^{-g} \prod_{l=1}^{g}\left(1-\alpha_{l}\right)\left(1-\bar{\alpha}_{l}\right)\left[\mathcal{O}_{\mathbb{C}^{2 g}}\right]
$$

In addition we have, see e.g., [M2, Section 14],

$$
\prod_{l=1}^{g}\left(1-\alpha_{l}\right)\left(1-\bar{\alpha}_{l}\right)=\# P i c^{0}(X)\left(\mathbb{F}_{q}\right)
$$

so that we get

$$
\left[\mathcal{O}_{\{0\}}\right]=q^{-g} \# P i c^{0}(X)\left(\mathbb{F}_{q}\right)\left[\mathcal{O}_{\mathbb{C}^{2 g}}\right]
$$

Theorem 3.1. The assignment $z^{d}\left[\mathcal{O}_{\{0\}}\right] \mapsto \mathbf{1}_{1, d}^{s s}, d \in \mathbb{Z}$, extends to an $R$-algebra anti-isomorphism $\Theta_{R}: \overline{\mathbf{C}} \rightarrow \dot{\mathbf{U}}_{R}^{>}$such that $\Theta_{R}\left(\pi_{n}(u) x\right)=u \bullet \Theta_{R}(x)$ for $x \in \overline{\mathbf{C}}_{n}$ and $u \in \mathbf{U}_{R}^{0}$.

Proof. It easily follows from the definitions of shuffle algebras that the identity map is an algebra anti-isomorphism $I d: \mathbf{A}_{R, \tilde{\zeta}(z)} \stackrel{\sim}{\rightarrow} \mathbf{A}_{R, \tilde{\zeta}\left(z^{-1}\right)}$. We consider the map $I d^{\prime}$ defined by

$$
I d_{\mid \mathbf{A}_{n}}^{\prime}=\frac{1}{\prod_{l=1}^{g}\left(1-x_{l}\right)^{n}\left(1-y_{l}\right)^{n}} I d_{\mathbf{A}_{n}}=\frac{q^{n g}}{\#\left(\operatorname{Pic}^{0}(X)\left(\mathbb{F}_{q}\right)\right)^{n}} I d_{\mathbf{A}_{n}} .
$$

The theorem is now a consequence of the chain of maps

$$
\overline{\mathbf{C}} \stackrel{\Phi}{\longrightarrow} \mathbf{A}_{R, \tilde{\zeta}(z)} \stackrel{I d^{\prime}}{\longrightarrow} \mathbf{A}_{R, \tilde{\zeta}\left(z^{-1}\right)} \stackrel{\dot{\Upsilon}}{\longleftarrow} \dot{\mathbf{U}}_{R}^{>}
$$

see Corollary 1.17 and Theorem 2.9. Note that all these maps are compatible with the relevant $R_{G L_{n}}$-actions.

Let $\Theta_{K}: \overline{\mathbf{C}}_{K} \rightarrow \dot{\mathbf{U}}_{K}^{>}$be the extension of scalars of $\Theta_{R}$ from $R$ to $K$. It is a $K$-algebra isomorphism.

Remarks 3.2. (a) The renormalization above is made so that $\Theta_{R}$ is compatible with the geometric class field theory.

(b) The reader might wonder why $\Theta$ is an anti-isomorphism rather than an isomorphism. This is only a consequence of our convention concerning the order of the multiplication in the Hall algebra, which follows the tradition in that field (see e.g., [R1], [S1]). Of course, had we considered the Hall algebra with the opposite multiplication (as it is done in $[\mathrm{K}]$, for instance) we would have obtained an honest isomorphism. 
3.2. Now we discuss the preimage, under the correspondence $\Theta_{R}$, of the constant function $\mathbf{1}_{r}^{\text {vec }}$ on the set $\operatorname{Bun}_{r}(X)$ of all vector bundles of rank $r$ over $X$. The element

$$
\mathbf{1}_{r}^{\text {vec }}=\sum_{\operatorname{rank}(\mathcal{V})=r} 1_{\mathcal{V}}
$$

strictly speaking does not belong to $\mathbf{H}_{X}$ since it is an infinite sum. We may break it up into terms according to the degree as $\mathbf{1}_{r}^{\mathbf{v e c}}=\sum_{d} \mathbf{1}_{r, d}^{\mathbf{v e c}}$. Each $\mathbf{1}_{r, d}^{\mathbf{v e c}}$ is still an infinite sum, but this sum belongs to the standard completion $\widehat{\mathbf{H}}_{X}$ of $\mathbf{H}_{X}$ defined as

$$
\widehat{\mathbf{H}}_{X}=\bigoplus_{r, d} \widehat{\mathbf{H}}_{X}[r, d], \quad \widehat{\mathbf{H}}_{X}[r, d]=\left\{f: \mathcal{I}_{r, d}^{v e c} \rightarrow \mathbb{C}\right\}=\prod_{\mathcal{F} \in \mathcal{I}_{r, d}^{v e c}} \mathbb{C} 1_{\mathcal{F}}
$$

Here $\mathcal{I}_{r, d}^{v e c}$ is the set of isomorphism classes of vector bundles over $X$ of rank $r$ and degree $d$. The completion $\widehat{\mathbf{H}}_{X}$ is still an algebra, see e.g., [BS, Section 2].

3.3. Let us begin with a heuristic computation. By [S1, Lemma 1.7] we have

$$
\Delta\left(\mathbf{1}_{\gamma}\right)=\sum_{\alpha+\beta=\gamma} v^{\langle\alpha, \beta\rangle} \mathbf{1}_{\alpha} \otimes \mathbf{1}_{\beta}, \quad \gamma \in \mathbb{Z}^{2} .
$$

Recall that $v=q^{-1 / 2}$. Iterating this and restricting to vector bundles, we get the following expression for the constant term of $\mathbf{1}_{r, d}^{\mathrm{vec}}$

$$
\begin{aligned}
J_{r}\left(\mathbf{1}_{r, d}^{\mathbf{v e c}}\right) & =\sum_{d_{1}+\cdots+d_{r}=d} v^{\sum_{i<j}\left\langle\left(1, d_{i}\right),\left(1, d_{j}\right)\right\rangle} \mathbf{1}_{1, d_{1}}^{\mathbf{s s}} \otimes \cdots \otimes \mathbf{1}_{1, d_{r}}^{\mathbf{s s}} \\
& =v^{r(r-1)(1-g) / 2} \sum_{d_{1}+\cdots+d_{r}=d} v^{(1-r) d_{1}+(3-r) d_{2}+\cdots+(r-1) d_{r}} \mathbf{1}_{1, d_{1}}^{\mathbf{s s}} \otimes \cdots \otimes \mathbf{1}_{1, d_{r}}^{\mathbf{s s}} .
\end{aligned}
$$

Using the identification $\mathbf{U}_{R}^{>}[1]^{\otimes r} \simeq R\left[z_{1}^{ \pm 1}, \ldots, z_{r}^{ \pm 1}\right]$ we may write the generating function

$$
\begin{aligned}
\sum_{d} J_{r}\left(\mathbf{1}_{r, d}^{\mathbf{v e c}}\right) s^{d} & =v^{c} \sum_{d_{1}, \ldots, d_{r}} v^{(1-r) d_{1}+(3-r) d_{2}+\cdots+(r-1) d_{r}} z_{1}^{d_{1}} \cdots z_{r}^{d_{r}} s^{\sum d_{i}} \\
& =v^{c}\left(\sum_{d_{1}}\left(v^{1-r} s z_{1}\right)^{d_{1}}\right) \cdots\left(\sum_{d_{r}}\left(v^{r-1} s z_{r}\right)^{d_{r}}\right) \\
& =v^{c} \delta\left(v^{1-r} s z_{1}\right) \cdots \delta\left(v^{r-1} s z_{r}\right)
\end{aligned}
$$

where $c=r(r-1)(1-g) / 2$ and where $\delta(z)=\sum_{d \in \mathbb{Z}} z^{d}$. Recall that the map

$$
\dot{\Upsilon}_{R}: \dot{\mathbf{U}}_{R}^{>} \rightarrow \mathbf{A}_{R, \tilde{\zeta}\left(z^{-1}\right)}
$$

is, in degree $r$, the composition of the constant term map $J_{r}$ with the multiplication by

$$
\tilde{\zeta}\left(z_{1}^{-1}, \ldots, z_{r}^{-1}\right)=\prod_{i<j}\left(1-z_{j} / z_{i}\right)^{-1} \prod_{i<j}\left\{\left(1-v^{2} z_{j} / z_{i}\right) \prod_{l=1}^{g}\left(1-\alpha_{l} z_{j} / z_{i}\right)\left(1-\bar{\alpha}_{l} z_{j} / z_{i}\right)\right\} .
$$

Multiplying formally the right hand side of (3.4) by $\tilde{\zeta}\left(z_{1}^{-1}, \ldots, z_{r}^{-1}\right)$ yields

$$
\Theta_{R}^{-1}\left(\mathbf{1}_{r, d}^{\text {vec }}\right)=v^{c} \delta\left(v^{1-r} s z_{1}\right) \cdots \delta\left(v^{r-1} s z_{r}\right) \tilde{\zeta}\left(z_{1}^{-1}, \ldots, z_{r}^{-1}\right)=v^{c} \tilde{\zeta}\left(v^{1-r}, v^{3-r}, \ldots, v^{r-1}\right)=0 .
$$

Thus one would be tempted to directly conclude that $\Theta_{R}^{-1}\left(\mathbf{1}_{r, d}^{\text {vec }}\right)=0$ and hence $\Theta_{R}^{-1}\left(\mathbf{1}_{r}^{\text {vec }}\right)=0$. Of course the above computation is not valid as such since it involves divergent sums. 


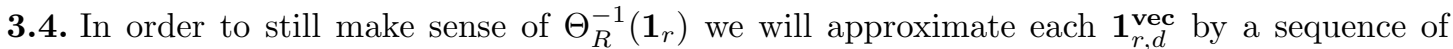
genuine elements of $\mathbf{H}_{X}$. For this we will use the Harder-Narasimhan filtration on coherent sheaves over $X$. We refer the reader to [S2] for the precise definitions, and for some of the results stated below. We denote by $H N(\mathcal{F})=\left(\alpha_{1}, \alpha_{2}, \ldots, \alpha_{s}\right)$ the Harder-Narasimhan type of a coherent sheaf $\mathcal{F}$. Recall that

$$
\begin{gathered}
\alpha_{1}, \alpha_{2}, \ldots, \alpha_{s} \in \mathbb{Z}^{2,+}, \quad \mu\left(\alpha_{1}\right)<\mu\left(\alpha_{2}\right)<\cdots<\mu\left(\alpha_{s}\right), \\
\mathbb{Z}^{2,+}=\left\{(r, d) \in \mathbb{Z}^{2} ; r \geqslant 1, \text { or } r=0, d \geqslant 1\right\}, \quad \mu(r, d)=d / r \in \mathbb{Q} \cup\{\infty\} .
\end{gathered}
$$

We write $\mathbf{1}_{r, d}^{\text {ss }}$ for the characteristic function of the set of semistable coherent sheaves of rank $r$ and degree $d$. We have

$$
\mathbf{1}_{r, d}^{\mathrm{vec}}=\sum_{\underline{\alpha} \in Y_{\alpha}} v^{\sum_{i<j}\left\langle\alpha_{i}, \alpha_{j}\right\rangle} \mathbf{1}_{\alpha_{1}}^{\mathrm{ss}} \cdots \mathbf{1}_{\alpha_{s}}^{\mathrm{ss}},
$$

where $Y_{r, d}$ is the set of all Harder-Narasimhan types $\underline{\alpha}=\left(\alpha_{1}, \ldots, \alpha_{s}\right)$ of weight $\sum_{i} \alpha_{i}=(r, d)$ for which $\mu\left(\alpha_{s}\right)<\infty$. By [S2, Theorem 2.4] each $\mathbf{1}_{\beta}^{\text {ss }}$ belongs to $\mathbf{U}_{X}^{>}$. So $\mathbf{1}_{r, d}$ is in the completion $\widehat{\mathbf{U}}_{X}^{>}$of $\mathbf{U}_{X}^{>}$. We approximate $\mathbf{1}_{r, d}^{\text {vec }}$ by partial sums of (3.5). For any finite subset $Z$ of $Y_{r, d}$, set

$$
\mathbf{1}_{r, d}^{Z}=\sum_{\underline{\alpha} \in Z} v^{\sum_{i<j}\left\langle\alpha_{i}, \alpha_{j}\right\rangle} \mathbf{1}_{\alpha_{1}}^{\mathrm{ss}} \cdots \mathbf{1}_{\alpha_{l}}^{\mathrm{ss}}
$$

We consider the following notion of convergence for a sequence of elements of $\overline{\mathbf{C}}$. Define a $\mathbb{Z}$ grading on the ring $R$ by setting $\operatorname{deg}\left(x_{i}\right)=\operatorname{deg}\left(y_{i}\right)=1$ for $i=1, \ldots, g$. The relation $x_{i} y_{i}=p$ in $R_{s}=R$ and the assignment $q \mapsto p^{-1}$ in (3.1) imply that $\operatorname{deg}(v)=1$. Write $R=\bigoplus_{l} R_{l}$ for the decomposition into graded pieces. We consider convergence with respect to the adic topology induced by this degree. More precisely, let us write

$$
R\left[z_{1}^{ \pm 1}, \ldots, z_{r}^{ \pm 1}\right]=\bigoplus_{l} R_{l}\left[z_{1}^{ \pm 1}, \ldots, z_{r}^{ \pm 1}\right], \quad R_{\geqslant l}\left[z_{1}^{ \pm 1}, \ldots, z_{r}^{ \pm 1}\right]=\bigoplus_{l^{\prime} \geqslant l} R_{l^{\prime}}\left[z_{1}^{ \pm 1}, \ldots, z_{r}^{ \pm 1}\right] .
$$

Then a sequence $\left(u_{i}\right)_{i \in I}$ of elements of $\mathbf{C}^{\prime} \subset R\left[z_{1}^{ \pm 1}, \ldots, z_{r}^{ \pm 1}\right]$ converges to $u$ if for any $l$ there exists $i_{0} \in I$ such that $u-u_{i} \in R_{\geqslant l}\left[z_{1}^{ \pm 1}, \ldots, z_{r}^{ \pm 1}\right]$ for any $i>i_{0}$.

Proposition 3.3. For any $(r, d)$, the sequence $\Theta_{R}^{-1}\left(\mathbf{1}_{r, d}^{Z}\right)$ converges to zero as $Z$ tends to $Y_{r, d}$.

Proof. Let us fix a pair $(r, d)$. The argument hinges on the following two lemmas, which are simple consequences of Corollary 1.10. Set $R_{[-n, n]}=\bigoplus_{l=-n}^{n} R_{l}$. Denote by $W \subset \mathbf{U}_{R}^{>}$the $\mathbb{C}$-subalgebra generated by $\left\{\mathbf{1}_{1, d}^{\text {ss }} ; d \in \mathbb{Z}\right\}$.

Lemma 3.4. (a) There exists $n \in \mathbb{N}$ such that for any $\underline{d}=\left(d_{1}, \ldots, d_{r}\right) \in \mathbb{Z}^{r}$ we have

$$
\Theta_{R}^{-1}\left(\mathbf{1}_{1, d_{1}}^{s s} \cdots \mathbf{1}_{1, d_{r}}^{s s}\right) \in R_{[-n, n]}\left[z_{1}^{ \pm 1}, \ldots, z_{r}^{ \pm 1}\right] .
$$

(b) There exists $n^{\prime} \in \mathbb{N}$ such that for $r^{\prime} \leqslant r$ and $d^{\prime} \in \mathbb{Z}$ we have

$$
\mathbf{1}_{r^{\prime}, d^{\prime}}^{s s} \in R_{-\left[n^{\prime}, n^{\prime}\right]} W .
$$

By Lemma 3.4(b) we have, for any HN type $\underline{\alpha}=\left(\alpha_{1}, \ldots, \alpha_{s}\right)$ of weight $(r, d)$

$$
\mathbf{1}_{\alpha_{1}}^{\mathrm{ss}} \cdots \mathbf{1}_{\alpha_{s}}^{\mathrm{ss}} \in R_{\left[-s n^{\prime}, s n^{\prime}\right]} W
$$

and using Lemma 3.4(a) we get

$$
\Theta_{R}^{-1}\left(\mathbf{1}_{\alpha_{1}}^{\mathbf{s s}} \cdots \mathbf{1}_{\alpha_{s}}^{\mathbf{s s}}\right) \in R_{-\left[s n^{\prime}-n, s n^{\prime}+n\right]}\left[z_{1}^{ \pm 1}, \ldots, z_{r}^{ \pm 1}\right] .
$$

From (3.5) and from the fact that $\sum_{i<j}\left\langle\alpha_{i}, \alpha_{j}\right\rangle \rightarrow \infty$ as the HN type $\underline{\alpha}$ goes to infinity, we deduce that the sequence $\Theta_{R}^{-1}\left(\mathbf{1}_{r, d}^{Z}\right)$ indeed converges in $R\left[z_{1}^{ \pm 1}, \ldots, z_{r}^{ \pm 1}\right]$ as $Z$ tends to $Y_{r, d}$. One shows, by the same calculation as in (3.4) that the limit is equal to zero. We leave the details to the reader. 
Remark 3.5. Proposition 3.3 says that in any lift of the isomorphism $\Theta_{R}$ to an equivalence of

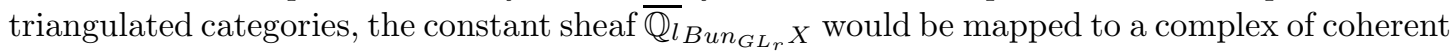
sheaves on the stack $\operatorname{Loc}_{G L_{r}} X$ whose class in the Grothendieck group is zero. As explained to us by $\mathrm{V}$. Lafforgue, this is indeed expected of the geometric Langlands correspondence: the constant sheaf $\overline{\mathbb{Q}}_{l_{B u n_{G L_{r}}} X}$ should be mapped to some unbounded acyclic complex in $D\left(\operatorname{Coh}\left(\operatorname{Loc}_{G L_{r}} X\right)\right)$.

3.5. Let us now fix some $r \geqslant 1$ and describe the image under our correspondence of the class $\left[\mathcal{O}_{\text {triv }_{r}}\right]=\left[\mathcal{O}_{\{0\}}\right]$ in $K^{T_{s} \times G L_{r}}\left(C_{\mathfrak{g l}_{r}}\right)$. Write $x \mathfrak{g}=\sum x_{l} \mathfrak{g}$ and $y \mathfrak{g}=\sum y_{l} \mathfrak{g}$. Let $i:\{0\} \rightarrow C_{\mathfrak{g l}_{r}}$ be the inclusion. We have

$$
L i^{*}\left(\left[\mathcal{O}_{\{0\}}\right]\right)=\Lambda_{-1}(x \mathfrak{g}+y \mathfrak{g})=\prod_{i, j=1}^{r} \kappa\left(z_{i} / z_{j}\right)=\prod_{l=1}^{g}\left(1-x_{l}\right)^{r}\left(1-y_{l}\right)^{r} \cdot \prod_{i \neq j} \kappa\left(z_{i} / z_{j}\right)
$$

where $\kappa(z)=\prod_{l=1}^{g}\left(1-x_{l} z\right)\left(1-y_{l} z\right)$. Next, recall the weighted symmetrization map $\Psi_{r}$ used in the definition of $\mathbf{A}_{\tilde{\zeta}(z)}$ in Section 1.10. Here $\tilde{\zeta}(z)$ is given by (1.21). A direct computation yields

$$
\begin{aligned}
\Psi_{r}\left(z_{1}^{r-1} z_{2}^{r-3} \cdots z_{r}^{1-r}\right. & \left.\cdot \prod_{i<j} \prod_{l=1}^{g}\left(1-x_{l}^{-1} z_{j} / z_{i}\right)\left(1-y_{l}^{-1} z_{j} / z_{i}\right)\right)= \\
= & (-1)^{r(r-1) / 2}[r] ! \prod_{i \neq j}^{g}\left(1-x_{l}^{-1} z_{i} / z_{j}\right)\left(1-y_{l}^{-1} z_{i} / z_{j}\right) \\
& =(-1)^{r(r-1) / 2}[r] ! q^{g r(r-1) / 2} \prod_{i \neq j} \kappa\left(z_{i} / z_{j}\right)
\end{aligned}
$$

which, up to a non-zero factor in $K$, is equal to $L i^{*}\left(\left[\mathcal{O}_{\{0\}}\right]\right)$. Here we have set

$$
[s]=1+q+\cdots+q^{s-1}, \quad[s] !=[1][2] \cdots[s] .
$$

This shows in particular that $\left[\mathcal{O}_{\{0\}}\right]$ belongs to the subalgebra $\overline{\mathbf{C}}_{K}$ of $K^{T_{s} \times G L_{r}}\left(C_{\mathfrak{g l}_{r}}\right) \otimes_{R} K$, and yields an expression for $\Theta_{K}\left(\left[\mathcal{O}_{\{0\}}\right]\right)$. In order to write this expression in a nice way, we introduce the following notation. Define a $K$-linear map

$$
\text { Ind }: K\left[z_{1}^{ \pm 1}, \ldots, z_{r}^{ \pm 1}\right] \rightarrow \dot{\mathbf{U}}_{K}^{>}, \quad z_{1}^{d_{1}} \cdots z_{r}^{d_{r}} \mapsto \mathbf{1}_{1, d_{1}}^{\mathrm{ss}} \cdots \mathbf{1}_{d_{r}}^{\mathrm{ss}} .
$$

If $\sigma=\left(\sigma_{1}, \ldots, \sigma_{r}\right) \in \mathbb{Z}^{r}$ we write $z^{\sigma}=z_{1}^{\sigma_{1}} \cdots z_{r}^{\sigma_{r}}$. We have the following formula.

Proposition 3.6. For $r \geqslant 1$ we have

$$
\Theta_{K}\left(\left[\mathcal{O}_{\text {triv }_{r}}\right]\right)=(-1)^{r(r-1) / 2} \frac{q^{-g r(r+1) / 2}}{[r] !} \operatorname{Ind}\left(z^{-2 \rho} \prod_{\sigma \in \Delta^{+}} \prod_{l=1}^{g}\left(1-\alpha_{l} z^{\sigma}\right)\left(1-\bar{\alpha}_{l} z^{\sigma}\right)\right)
$$

where $\Delta^{+} \subset \mathbb{Z}^{r}$ is the set of positive roots of $\mathfrak{g l}_{r}$.

Proof. This is a direct consequence of (3.7) and Theorem 3.1.

Remark 3.7. Proposition 3.6 is stated in terms of the multiplication in the twisted spherical Hall algebra $\dot{\mathbf{U}}_{K}^{>}$. If instead one uses the usual spherical Hall algebra $\mathbf{U}_{K}^{>}$then the expression for $\Theta_{K}\left(\left[\mathcal{O}_{\text {triv }_{r}}\right]\right)$ is a little bit more symmetric

$$
\Theta_{K}\left(\left[\mathcal{O}_{\text {triv }_{r}}\right]\right)=(-1)^{r(r-1) / 2} \frac{q^{-g r(r+1) / 2}}{[r] !} \operatorname{Ind}\left(z^{-2 g \rho} \prod_{\sigma \in \Delta^{+}} \prod_{l=1}^{g}\left(1-\alpha_{l} z^{\sigma}\right)\left(1-\bar{\alpha}_{l} z^{\sigma}\right)\right) .
$$


Example 3.8. Let us assume that $X$ is an elliptic curve and that $r=2$. A direct computation shows that, up to a global factor, the function $\Theta_{K}\left(\left[\mathcal{O}_{\text {triv }_{2}}\right]\right)$ takes the following non-zero values on the closed points of $\mathrm{Bun}_{2,0} \mathrm{X}$

$$
\Theta_{K}\left(\left[\mathcal{O}_{\text {triv }_{2}}\right]\right)\left(\mathcal{L}_{0} \oplus \mathcal{L}_{0}^{\prime}\right)=(1+q)\left(\# X\left(\mathbb{F}_{q}\right)-2\right)
$$

if $\mathcal{L}_{0}, \mathcal{L}_{0}^{\prime} \in P i c^{0} X, \mathcal{L}_{0} \neq \mathcal{L}_{0}^{\prime}$,

$$
\Theta_{K}\left(\left[\mathcal{O}_{\text {triv }_{2}}\right]\right)\left(\mathcal{L}_{0}^{(2)}\right)=(1+q)\left(\# X\left(\mathbb{F}_{q}\right)-1\right)
$$

if $\mathcal{L}_{0}^{(2)}$ is the (unique) indecomposable self-extension of some $\mathcal{L}_{0} \in P i c^{0} X$,

$$
\Theta_{K}\left(\left[\mathcal{O}_{\text {triv }_{2}}\right]\right)(\mathcal{V})=(1+q) \# X\left(\mathbb{F}_{q}\right)
$$

if $\mathcal{V}$ is a rank two stable bundle, and finally

$$
\Theta_{K}\left(\left[\mathcal{O}_{\text {triv }_{2}}\right]\right)\left(\mathcal{L}_{-1} \oplus \mathcal{L}_{1}\right)=q
$$

for $\mathcal{L}_{-1} \in \operatorname{Pic}^{-1} X, \mathcal{L}_{1} \in P i c^{1} X$. Thus $\Theta_{K}\left(\left[\mathcal{O}_{\text {triv }_{2}}\right]\right)$ is supported on the union of the two HarderNarasimhan stratas $S_{2,0}$ and $S_{(1,-1),(1,1)}$. It is easy to see, using e.g., (3.9), that for a curve $X$ of genus $g$ the function $\Theta_{K}\left(\left[\mathcal{O}_{\text {triv }_{2}}\right]\right)$ is supported on the union of Harder-Narasimhan stratas

$$
S_{(2,0)} \cup S_{(1,-1),(1,1)} \cup \cdots \cup S_{(1,-g),(1, g)} .
$$

More generally, if $\mathfrak{n}_{+} \subset \mathfrak{g l}_{r}$ is the positive nilpotent subalgebra and if $\Phi$ is the set of weights occuring in $\Lambda^{\bullet}\left(H^{1}\left(X, \overline{\mathbb{Q}}_{l}\right) \otimes \mathfrak{n}_{+}\right)$then $\Theta_{K}\left(\left[\mathcal{O}_{\operatorname{triv}_{r}}\right]\right)$ is supported on the union of Harder-Narasimhan strata whose type belongs to the convex hull of $\{\alpha-2 g \rho ; \alpha \in \Phi\}$.

\section{Acknowledgements.}

We would like to thank V. Lafforgue for useful conversations.

\section{APPENDICES}

\section{Appendix A. The principal Hall algebra}

To our knowledge, the Hall algebra $\mathbf{H}_{X}$ cannot be regarded as a shuffle algebra. However, there is a subalgebra of $\mathbf{H}_{X}$ which strictly contains $\mathbf{U}_{X}$ and which admits a similar description. It is the principal Hall algebra that we describe now.

A.1. We define the principal Hall algebra $\mathbf{H}_{X}^{p r}$ of $X$ as the subalgebra of $\mathbf{H}_{X}$ which is generated by $\mathbf{H}_{X}[0]$ and $\mathbf{H}_{X}$ [1], i.e., as the subalgebra of $\mathbf{H}_{X}$ generated by all the characteristic functions $1_{\mathcal{F}}$ for $\mathcal{F}$ a torsion sheaf or a line bundle. We have

$$
\mathbf{U}_{X} \subset \mathbf{H}_{X}^{p r} \subset \mathbf{H}_{X},
$$

and unless $X \simeq \mathbb{P}^{1}$ the inclusions are strict. Let $\mathbf{H}_{X}^{>, p r}$ be the subalgebra generated by the functions $1_{\mathcal{L}}$ for $\mathcal{L} \in \operatorname{Pic}(X)$. As for the spherical Hall algebra, we have

$$
\mathbf{H}_{X}^{p r} \simeq \mathbf{H}_{X}^{>, p r} \otimes \mathbf{H}_{X}[0] .
$$

The aim of this section is to give a realization of $\mathbf{H}_{X}^{>, p r}$ as a shuffle algebra. 
A.2. Let $\widehat{\operatorname{Pic}(X)}$ be the group of all complex characters $\chi: \operatorname{Pic}(X) \rightarrow \mathbb{C}^{\times}$. The group $\widehat{\operatorname{Pic}(X)}$ fits in a natural sequence

$$
1 \longrightarrow \mathbb{C}^{\times} \stackrel{\iota}{\longrightarrow} \widehat{\operatorname{Pic}(X)} \stackrel{r}{\longrightarrow} \widehat{\operatorname{Pic}(X)} \longrightarrow 1
$$

with

$$
\iota: \mathbb{C}^{\times} \rightarrow \widehat{\operatorname{Pic}(X)}, \quad z \mapsto\left(\mathcal{L} \mapsto z^{\operatorname{deg}(\mathcal{L})}\right),
$$

and with $r: \widehat{\operatorname{Pic}(X)} \rightarrow \widehat{P i c^{0}(X)}$ being the restriction. We will write $\rho \sim \chi$ for two characters satisfying $r(\rho)=r(\chi)$. For $d \in \mathbb{Z}$ and $\chi: \operatorname{Pic}(X) \rightarrow \mathbb{C}^{\times}$we set

$$
\mathbf{1}_{1, d}^{\chi}=\sum_{\mathcal{L} \in \operatorname{Pic}^{d}(X)} \chi(\mathcal{L}) 1_{\mathcal{L}}
$$

When $\chi=1$, the trivial character, we recover the function $\mathbf{1}_{1, d}^{\text {ss }}$ introduced in Section 1.6. We need to introduce certain elements of $\mathbf{H}_{T o r(X)}$. The determinant $\operatorname{Vec}(X) \rightarrow \operatorname{Pic}(X)$ factors to a morphism of abelian groups $K_{0}(X) \rightarrow \operatorname{Pic}(X)$. This allows to make sense of the determinant $\operatorname{det}(\mathcal{T}) \in \operatorname{Pic}^{d}(X)$ of a torsion sheaf $\mathcal{T}$ of degree $d$. Recall from Section 1.3 the elements $\mathbf{1}_{0, l}, T_{0, l}$ and $\theta_{0, l}$ in $H_{\operatorname{Tor}(X)}$. For $\chi \in \widehat{\operatorname{Pic}(X)}$ and $l \geqslant 0$ we set

$$
\begin{gathered}
\mathbf{1}_{0, d}^{\chi}=\sum_{\substack{\mathcal{T} \in \operatorname{Tor}(X) \\
\operatorname{deg}(\mathcal{T})=d}} \chi(\operatorname{det}(\mathcal{T})) 1_{\mathcal{T}}, \\
T_{0, d}^{\chi}=\sum_{\mathcal{T} \in \operatorname{Tor}(X)} \chi(\operatorname{det}(\mathcal{T})) T_{0, d}(\mathcal{T}) 1_{\mathcal{T}}, \\
\theta_{0, l}^{\chi}=\sum_{\mathcal{T} \in \operatorname{Tor}(X)} \chi(\operatorname{det}(\mathcal{T})) \theta_{0, l}(\mathcal{T}) 1_{\mathcal{T}} .
\end{gathered}
$$

Using the additivity of the determinant one easily checks that as before

$$
1+\sum_{d \geqslant 1} \mathbf{1}_{0, d}^{\chi} s^{d}=\exp \left(\sum_{d} \frac{T_{0, d}^{\chi}}{[d]} s^{d}\right), \quad 1+\sum_{d \geqslant 1} \theta_{0, d}^{\chi} s^{d}=\exp \left(\left(v^{-1}-v\right) \sum_{d} T_{0, d}^{\chi} s^{d}\right) .
$$

A.3. The elements $\mathbf{1}_{1, n}^{\chi}, \theta_{0, d}^{\chi}$, etc, introduced above satisfy properties very similar to those of the elements $\mathbf{1}_{1, n}^{\mathbf{s s}}, \theta_{0, d}$, etc. We summarize these properties in the next few lemmas.

Lemma A.1. The following holds

(a) $\widetilde{\Delta}\left(\theta_{0, d}^{\chi}\right)=\sum_{l=0}^{d} \theta_{0, l}^{\chi} \boldsymbol{\kappa}_{0, d-l} \otimes \theta_{0, d-l}^{\chi}$,

(b) $\widetilde{\Delta}\left(T_{0, d}^{\chi}\right)=T_{0, d}^{\chi} \otimes 1+\kappa_{0, d} \otimes T_{0, d}^{\chi}$.

Proof. We prove $(a)$. From Lemma $1.1(b)$ we get

$$
\widetilde{\Delta}\left(\theta_{0, d}^{\mathcal{K}}\right)=\sum_{l=0}^{d} \sum_{\mathcal{L}, \mathcal{L}^{\prime}} \theta_{0, l}^{1, \mathcal{L}} \boldsymbol{\kappa}_{0, d-l} \otimes \theta_{0, d-l}^{1, \mathcal{L}^{\prime}}
$$

where the sum ranges over the pairs $\mathcal{L} \in \operatorname{Pic}^{d}(X), \mathcal{L}^{\prime} \in \operatorname{Pic}^{d-l}(X)$ satisfying $\mathcal{L}+\mathcal{L}^{\prime}=\mathcal{K}$. Thus

$$
\begin{aligned}
\widetilde{\Delta}\left(\theta_{0, d}^{\chi, \mathcal{K}}\right)=\chi(\mathcal{K}) \widetilde{\Delta}\left(\theta_{0, d}^{\chi, \mathcal{K}}\right) & =\sum_{l=0}^{d} \sum_{\mathcal{L}, \mathcal{L}^{\prime}} \chi(\mathcal{L}) \theta_{0, l}^{1, \mathcal{L}} \boldsymbol{\kappa}_{0, d-l} \otimes \chi\left(\mathcal{L}^{\prime}\right) \theta_{0, d-l}^{1, \mathcal{L}^{\prime}} \\
& =\sum_{l=0}^{d} \sum_{\mathcal{L}, \mathcal{L}^{\prime}} \theta_{0, l}^{\chi, \mathcal{L}} \boldsymbol{\kappa}_{0, d-l} \otimes \theta_{0, d-l}^{\chi, \mathcal{L}^{\prime}} .
\end{aligned}
$$

Summing over all $\mathcal{K}$ yields $(a)$. The proof of $(b)$ is entirely similar. 
Lemma A.2. For any $\chi \in \widehat{\operatorname{Pic}(X)}$ we have

$$
\widetilde{\Delta}\left(\mathbf{1}_{1, d}^{\chi}\right)=\mathbf{1}_{1, d}^{\chi} \otimes 1+\sum_{l \geqslant 0} \theta_{0, l}^{\chi} \boldsymbol{\kappa}_{1, d-l} \otimes \mathbf{1}_{1, d-l}^{\chi} .
$$

Proof. For any $\mathcal{K} \in \operatorname{Pic}^{l}(X)$ let us denote by $\theta_{0, l}^{\chi, \mathcal{K}}$ the projection of $\theta_{0, l}^{\chi}$ to the subset of torsion sheaves of determinant $\mathcal{K}$. From (1.4) we immediately deduce, for any fixed line bundle $\mathcal{L} \in$ $\operatorname{Pic}^{d}(X)$

$$
\widetilde{\Delta}\left(1_{\mathcal{L}}\right)=1_{\mathcal{L}} \otimes 1+\sum_{l \geqslant 0} \theta_{0, l}^{1, \mathcal{L}-\mathcal{L}^{\prime}} \boldsymbol{\kappa}_{1, d-l} \otimes 1_{\mathcal{L}^{\prime}}
$$

Summing over $\mathcal{L}$ this yields

$$
\begin{aligned}
\widetilde{\Delta}\left(\mathbf{1}_{1, d}^{\chi}\right) & =\mathbf{1}_{1, d}^{\chi} \otimes 1+\sum_{l \geqslant 0} \sum_{\substack{\mathcal{L} \in \operatorname{Pic}^{d}(X) \\
\mathcal{L}^{\prime} \in P i c^{d-l}(X)}} \chi(\mathcal{L}) \theta_{0, l}^{1, \mathcal{L}-\mathcal{L}^{\prime}} \boldsymbol{\kappa}_{1, d-l} \otimes 1_{\mathcal{L}^{\prime}} \\
& =\mathbf{1}_{1, d}^{\chi} \otimes 1+\sum_{l \geqslant 0} \sum_{\substack{\mathcal{L} \in P i c^{d}(X) \\
\mathcal{L}^{\prime} \in P i c^{d-l}(X)}} \chi\left(\mathcal{L}-\mathcal{L}^{\prime}\right) \chi\left(\mathcal{L}^{\prime}\right) \theta_{0, l}^{1, \mathcal{L}-\mathcal{L}^{\prime}} \boldsymbol{\kappa}_{1, d-l} \otimes 1_{\mathcal{L}^{\prime}} \\
& =\mathbf{1}_{1, d}^{\chi} \otimes 1+\sum_{l \geqslant 0} \sum_{\substack{\mathcal{L} \in P i c^{d}(X) \\
\mathcal{L}^{\prime} \in P i c^{d-l}(X)}} \theta_{0, l}^{\chi, \mathcal{L}-\mathcal{L}^{\prime}} \boldsymbol{\kappa}_{1, d-l} \otimes \chi\left(\mathcal{L}^{\prime}\right) 1_{\mathcal{L}^{\prime}} \\
& =\mathbf{1}_{1, d}^{\chi} \otimes 1+\sum_{l \geqslant 0} \theta_{0, l}^{\chi} \boldsymbol{\kappa}_{1, d-l} \otimes \mathbf{1}_{1, d-l}^{\chi} .
\end{aligned}
$$

Consider the zeta function of $\chi$ defined as

$$
\zeta_{X}^{\chi}(z)=\exp \left(\sum_{d \geqslant 1} \sum_{\substack{l \mid d \\ x \in Y_{l}}} \chi\left(\operatorname{det}\left(\mathcal{O}_{x}\right)\right) \frac{l}{d} z^{d}\right),
$$

where $Y_{l}$ stands for the set of closed points of $X$ of degree $l$. When $\chi=1$ we have of course $\zeta_{X}^{\chi}=\zeta_{X}$. It is known that when $\chi \nsim 1$ the function $\zeta_{X}^{\chi}$ is a polynomial of degree $2 g-2$.

Lemma A.3. We have

(a) $\left(\mathbf{1}_{1, n}^{\chi}, \mathbf{1}_{1, n}^{\rho}\right)_{G}=0$ unless $\rho \sim \chi$, and $\left\langle\mathbf{1}_{1, n}^{\chi}, \mathbf{1}_{1, n}^{\chi}\right\rangle=\# P i c^{0}(X) /(q-1)$.

(b) $\left(T_{0, d}^{\chi}, T_{0, d}^{\rho}\right)_{G}=\frac{v^{d}[d]}{v^{-1}-v} \sum_{l \mid d} \sum_{x \in Y_{d}} \chi \bar{\rho}\left(\operatorname{det}\left(\mathcal{O}_{x}\right)\right) \frac{l}{d}$.

Proof. Statement $(a)$ follows directly from the definitions and from the orthogonality property of characters. The proof of statement $(b)$ is an easy modification of Lemma $1.1(c)$.

Recall the Hecke action, see Section 1.5,

$$
\text { - : } \mathbf{H}_{T o r(X)} \otimes \mathbf{H}_{V e c(X)} \rightarrow \mathbf{H}_{V e c(X)}, \quad f \otimes g \mapsto \omega(f \cdot g) .
$$

Lemma A.4. There are complex numbers $\xi_{d}^{\sigma}$ for $\sigma \in \widehat{\operatorname{Pic}(X)}$ and $d \geqslant n$ such that for any two characters $\chi, \rho$ and any $d \geqslant 0$ we have

$$
\theta_{0, d}^{\chi} \bullet \mathbf{1}_{1, n}^{\rho}=\xi_{d}^{\chi \bar{\rho}} \mathbf{1}_{1, n+d}^{\rho} .
$$

Moreover we have

$$
\theta_{0, d}^{\chi} \mathbf{1}_{1, n}^{\rho}=\sum_{l=0}^{d} \zeta_{l}^{\chi \bar{\rho}} \mathbf{1}_{1, n+l}^{\rho} \theta_{0, d-l}^{\chi}
$$


and, as a series in $\mathbb{C}[[s]]$,

$$
\sum_{d \geqslant 0} \xi_{d}^{\chi} s^{d}=\frac{\zeta_{X}^{\chi}(s)}{\zeta^{\chi}\left(q^{-1} s\right)}
$$

Proof. Since $T_{0, d}^{\chi}$ is primitive, i.e., $\Delta\left(T_{0, d}^{\chi}\right)=T_{0, d}^{\chi} \otimes 1+1 \otimes T_{0, d}^{\chi}$, we have

$$
\left[T_{0, d}^{\chi}, \mathbf{1}_{1, n}^{\rho}\right]=T_{0, d}^{\chi} \bullet \mathbf{1}_{1, n}^{\rho} \in \mathbf{H}_{V e c(X)}[1, n+d]=\bigoplus_{\sigma} \mathbb{C} \mathbf{1}_{1, n+d}^{\sigma},
$$

where the sum ranges over a complete set of inequivalent characters $\sigma \in \widehat{\operatorname{Pic}(X)}$. Let us write $\left[T_{0, d}^{\chi}, \mathbf{1}_{1, n}^{\rho}\right]=\sum_{\sigma} u_{\chi, \rho}^{\sigma} \mathbf{1}_{1, n+d}^{\sigma}$ for some scalars $u_{\chi, \rho}^{\sigma}$. We will prove that $u_{\chi, \rho}^{\sigma}=0$ unless $\sigma$ is equivalent to $\rho$. Indeed, if $\sigma \not \rho$ then

$$
\left(\left[T_{0, d}^{\chi}, \mathbf{1}_{1, n}^{\rho}\right], \mathbf{1}_{1, n+d}^{\sigma}\right)_{G}=u_{\chi, \rho}^{\sigma}\left(\mathbf{1}_{1, n+d}^{\sigma}, \mathbf{1}_{1, n+d}^{\sigma}\right)_{G}
$$

while on the other hand by Lemma A.3 (a)

$$
\begin{aligned}
\left(\left[T_{0, d}^{\chi}, \mathbf{1}_{1, n}^{\rho}\right], \mathbf{1}_{1, n+d}^{\sigma}\right)_{G} & =\left(T_{0, d}^{\chi} \mathbf{1}_{1, n}^{\rho}, \mathbf{1}_{1, n+d}^{\sigma}\right)_{G} \\
& =\left(T_{0, d}^{\chi} \otimes \mathbf{1}_{1, n}^{\rho}, \widetilde{\Delta}\left(\mathbf{1}_{1, n+d}^{\sigma}\right)\right)_{G}=\left(T_{0, d}^{\chi}, \theta_{0, d}^{\sigma}\right)_{G}\left(\mathbf{1}_{1, n}^{\rho}, \mathbf{1}_{1, n}^{\sigma}\right)_{G}=0 .
\end{aligned}
$$

It follows that $\left[T_{0, d}^{\chi}, \mathbf{1}_{1, n}^{\rho}\right] \in \mathbb{C} \mathbf{1}_{1, n+d}^{\rho}$. Using (A.2) we deduce that $\theta_{0, d}^{\chi} \bullet \mathbf{1}_{1, n}^{\rho} \in \mathbb{C} \mathbf{1}_{1, n+d}^{\rho}$ as wanted. Statement (A.4) is a consequence of Lemma A.1 (a). To prove (A.5), note that by the above calculation together with Lemma A.3 (b) we have

$$
T_{0, d}^{\chi} \bullet \mathbf{1}_{1, n}^{\rho}=v^{d}[d] \sum_{l \mid d} \sum_{x \in Y_{d}} \chi \bar{\rho}\left(\operatorname{det}\left(\mathcal{O}_{x}\right)\right) \frac{l}{d} \cdot \mathbf{1}_{1, n+d}^{\rho},
$$

from which it entails

$$
\begin{aligned}
\sum_{d \geqslant 0} \xi_{d}^{\chi \bar{\rho}} s^{d} & =\exp \left(\left(v^{-1}-v\right) \sum_{d \geqslant 1} v^{d}[d] \sum_{l \mid d} \sum_{x \in Y_{d}} \chi \bar{\rho}\left(\operatorname{det}\left(\mathcal{O}_{x}\right)\right) \frac{l}{d} s^{d}\right) \\
& =\exp \left(\sum_{d \geqslant 1}\left(1-q^{-d}\right) \sum_{l \mid d} \sum_{x \in Y_{d}} \chi \bar{\rho}\left(\operatorname{det}\left(\mathcal{O}_{x}\right)\right) \frac{l}{d} s^{d}\right) \\
& =\frac{\zeta_{X}^{\bar{\rho}}(s)}{\zeta^{\chi \bar{\rho}}\left(q^{-1} s\right)}
\end{aligned}
$$

Lemma A.4 is proved.

A.4. We may use the constant term map and the above Lemmas A.1-A.4 to get a combinatorial realization of $\mathbf{H}_{X}^{>, p r}$ as a shuffle algebra. We describe this realization below and leave the details of the proof to the reader. Let $\Xi$ be a set of representatives in $\widehat{\operatorname{Pic}(X)}$ for the equivalence classes $\widehat{\operatorname{Pic}(X)} / \operatorname{Im}(\iota)$, where $\iota$ is as in (A.1). We may (and we will) choose the elements of $\Xi$ to be unitary, i.e., $|\chi(\mathcal{L})|=1$ for any $\chi \in \Xi$ and $\mathcal{L} \in \operatorname{Pic}(X)$. Since the characters of a finite abelian group $B$ form a $\mathbb{C}$-basis of the set of all functions $B \rightarrow \mathbb{C}$, the collection of elements $\left\{\mathbf{1}_{1, d}^{\chi} ; d \in \mathbb{Z}, \chi \in \Xi\right\}$ forms a basis of $\mathbf{H}_{X}^{>}[1]$. Hence it generates $\mathbf{H}_{X}^{>, p r}$. For $\chi_{1}, \ldots, \chi_{r} \in \Xi$ let $\delta_{\chi_{1}, \ldots, \chi_{r}} \in \mathbb{C}\left[\Xi^{r}\right]$ be the characteristic function of $\left\{\left(\chi_{1}, \ldots, \chi_{r}\right)\right\}$. This allows us to write

$$
\mathbf{D}_{r}=\mathbb{C}\left[\Xi^{r} \times\left(\mathbb{C}^{\times}\right)^{r}\right]=\bigoplus_{\chi_{1}, \ldots, \chi_{r}} \bigoplus_{d_{1}, \ldots, d_{r}} \mathbb{C} \delta_{\chi_{1}, \ldots, \chi_{r}} x_{1}^{d_{1}} \cdots x_{r}^{d_{r}}, \quad \chi_{i} \in \Xi, \quad d_{i} \in \mathbb{Z} .
$$

To any $\chi \in \Xi$ we associate a function $g^{\chi}(z) \in \mathbb{C}(z)$ as follows. Let $\left\{\beta_{1}^{\chi}, \ldots, \beta_{2 g}^{\chi}\right\}$ be the Frobenius eigenvalues in $H^{1}\left(X \otimes \overline{\mathbb{F}_{q}}, \mathcal{L}_{\chi}\right)$ where $\mathcal{L}_{\chi}$ is the rank one local system on $X \otimes \overline{\mathbb{F}_{q}}$ associated with $\chi$. We have

$$
\zeta_{X}^{\chi}(z)=\prod_{i=1}^{2 g}\left(1-\beta_{i}^{\chi} z\right) /(1-z)(1-q z), \quad\left|\beta_{i}^{\chi}\right|=q^{1 / 2},
$$


see e.g., [D]. We define

$$
\gamma_{\chi}=q^{-1} \prod_{i=1}^{2 g} \beta_{i}^{\chi}=\prod_{l=0}^{2} \operatorname{det}\left(F r ; H^{i}\left(X \otimes \overline{\mathbb{F}_{q}}, \mathcal{L}_{\chi}\right)\right)^{(-1)^{i+1}} .
$$

This factor enters into the functional equation

$$
\zeta^{\chi}\left(q^{-1} z\right)=\gamma_{\chi}^{-1} z^{2(g-1)} \zeta^{\bar{\chi}}\left(z^{-1}\right)
$$

Put

$$
\begin{gathered}
g^{\chi}(z)=\left(1-q z^{-1}\right)(1-q z) z^{g-1} \gamma_{\chi}^{-1 / 2} \zeta^{\chi}\left(z^{-1}\right), \\
g=\sum_{\chi_{1}, \chi_{2}} \delta_{\chi_{1}, \chi_{2}} g^{\chi_{1} \overline{\chi_{2}}}\left(x_{1} / x_{2}\right) \in \mathbf{D}_{2} .
\end{gathered}
$$

For any pair $(i, j) \subset\{1, \ldots, r\}$ we set also

$$
g_{i, j}=\sum_{\chi_{1}, \ldots, \chi_{r}} \delta_{\chi_{1}, \ldots, \chi_{r}} g^{\chi_{i} \overline{\chi_{j}}}\left(x_{i} / x_{j}\right)
$$

Finally, we put $g\left(x_{1}, \ldots, x_{r}\right)=\prod_{i<j} g_{i, j} \in \mathbf{D}_{r}$. We are now ready to define our shuffle algebra. Consider the weighted symmetrization operator

$$
\Psi_{r}: \mathbf{D}_{r} \rightarrow \mathbf{D}_{r}, \quad f\left(x_{1}, \ldots, x_{r}\right) \mapsto \sum_{\sigma \in \mathfrak{S}_{r}} \sigma\left(f\left(x_{1}, \ldots, x_{r}\right) g\left(x_{1}, \ldots, x_{r}\right)\right) .
$$

Let $\mathbf{A}_{r} \subset \mathbf{D}_{r}$ be the image of $\Psi_{r}$ and set $\mathbf{A}^{p r}=\bigoplus_{r \geqslant 0} \mathbf{A}_{r}$. As in the context of Section 1.9 there exists a unique associative algebra structure on $\mathbf{A}^{\text {pr }}$ fitting in the commutative diagram

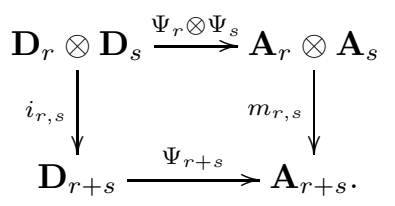

Proposition A.5. The assignement $\mathbf{1}_{1, l}^{\chi} \mapsto \delta_{\chi} x^{l} \in \mathbf{A}_{1}$ extends to an algebra isomorphism $\Upsilon_{X}^{p r}: \mathbf{H}_{X}^{>, p r} \stackrel{\sim}{\rightarrow} \mathbf{A}^{p r}$.

Note that unlike for the spherical Hall algebra $\mathbf{U}_{X}$, there are no rational forms $\mathbf{H}_{R}^{p r}$ of $\mathbf{H}_{X}^{p r}$ : the principal Hall algebra depends on more than just the set $\left\{\alpha_{1}, \ldots, \bar{\alpha}_{g}\right\}$ of Weil numbers of $X$; it depends on the group stucture of $\operatorname{Pic}^{0}(X)$ as well.

\section{Appendix B. Generalization to arbitrary Reductive groups}

The isomorphism $\Theta$, when restricted to a given rank $r$, provides a Langlands isomorphism between a subspace $\mathbf{C}_{r}^{\prime}$ of the equivariant K-theory $K^{T_{s} \times G L_{r}}\left(C_{\mathfrak{g l}_{r}}\right)$ and the spherical component $\mathbf{U}_{X}^{>}[r]$ of $\mathbf{H}_{X}^{>}[r]$, which can be regarded as the space of functions on $B u n_{r} X=B_{u n_{G L_{r}}} X$ which are induced from locally constant function on $B u n_{H} X$. Here $H \subset G L_{r}$ is a maximal torus. This isomorphism may be generalized to an arbitrary algebraic reductive group $G$ in place of $G L_{r}$. Indeed, the right hand side of $\Theta$, i.e., the spherical component of $F u n\left(\operatorname{Bun}_{G} X\right)$, may be defined in general and described using the Gindikin-Karpelevich formula, while the left hand side makes sense for an arbitrary reductive group $G^{\vee}$ as well and may be computed using the method of Section 2 . We briefly explain this in the present section. 
B.1. Let $G$ be a reductive algebraic group defined over $\mathbb{F}_{q}$. Let $F=\mathbb{F}_{q}(X)$ be the function field of $X$. For any closed point $x \in X$ we denote by $\widehat{O}_{x}$ the completed local ring at $x$ and by $F_{x}$ is field of fractions. Let $\mathbb{A}_{X}$, resp. $\mathbb{O}_{X}$ be the ring of adèles, resp. integer adèles of $X$. Following Weil, we identify the set $\operatorname{Bun}_{G} X$ of $G$-bundles over $X$ with a double quotient

$$
\operatorname{Bun}_{G} X \simeq G(F) \backslash G\left(\mathbb{A}_{X}\right) / G\left(\mathbb{O}_{X}\right) .
$$

Let $B \subset G$ be a Borel subgroup and let $H=B / U$ be the Levi factor (a torus). The sets of $H$-bundles and $B$-bundles over $X$ are

$$
\operatorname{Bun}_{B} X \simeq B(F) \backslash B\left(\mathbb{A}_{X}\right) / B\left(\mathbb{O}_{X}\right), \quad \operatorname{Bun}_{H} X \simeq H(F) \backslash H\left(\mathbb{A}_{X}\right) / H\left(\mathbb{O}_{X}\right) .
$$

Let $A F_{X, G}$ be the set of compactly supported functions $f: B u n_{G} X \rightarrow \mathbb{C}$. We define $A F_{X, H}$ in a similar way. The natural inclusion and projection $B \rightarrow G, B \rightarrow H$ induce maps

$$
\text { Bun }_{H} X \stackrel{p_{1}}{\longleftarrow} \text { Bun }_{B} X \stackrel{p_{2}}{\longrightarrow} \text { Bun }_{G} X .
$$

The map $p_{1}$ is smooth. We define the (Eisenstein) induction map as

$$
\operatorname{Ind}_{H}^{G}: A F_{X, H} \rightarrow A F_{X, G}, \quad f \mapsto p_{1 !} p_{2}^{*}(f),
$$

and the constant term map as

$$
\operatorname{Res}_{G}^{H}: A F_{X, G} \rightarrow F u n\left(\operatorname{Bun}_{H} X, \mathbb{C}\right), \quad g \mapsto p_{2 !} p_{1}^{*}(g) .
$$

For $G=G L_{n}$ we have $A F_{X, G}=\mathbf{H}_{X}^{>}[n], A F_{X, H}=\left(\mathbf{H}_{X}^{>}[1]\right)^{\otimes n}$ and the induction/restriction maps correspond to the product/coproduct in the Hall algebra of $X$.

B.2. The connected components of $B u n_{H} X$ are parametrized by elements of the lattice of cocharacters $P^{\vee}=H o m\left(\mathbb{C}^{\times}, H\right)$. To $\lambda^{\vee} \in P^{\vee}$ we associate the connected component of the $H$-bundle $\mathcal{F}_{\lambda \vee}$ induced from some line bundle $\mathcal{F} \in P i c^{1}(X)$ by the map $\lambda^{\vee}: \mathbb{C}^{\times} \rightarrow H$. Let $1_{\lambda \vee} \in A F_{X, H}$ be the characteristic function of the connected component of $\mathcal{F}_{\lambda^{\vee}}$. Let

$$
\mathbf{U}_{X, H} \subset A F_{X, H}
$$

be the space of locally constant functions. It is identified with the group algebra $\mathbb{C}\left[P^{\vee}\right]$ in the obvious way. Let $\Delta^{\vee} \subset P^{\vee}$ be the coroot system of $G$, and let $\Delta^{\vee,+}$ be the set of positive coroots corresponding to $B$. We likewise define $P^{\vee},+$ to be the positive cone in $P^{\vee}$. Let $\widehat{\mathbf{U}}_{X, H}=\widehat{\mathbb{C}\left[P^{\vee}\right]}$ be the formal completion of $\mathbf{U}_{X, H}=\mathbb{C}\left[P^{\vee}\right]$ in the direction of $P^{\vee},+$. An element of $\widehat{\mathbf{U}}_{X, H}$ is a formal linear combination $\sum_{\lambda \vee} a_{\lambda \vee} 1_{\lambda \vee}$ whose support lies in a finite union of translates of $P^{\vee,+}$. For $s(z) \in \mathbb{C}(z)$ a rational function and $\mu^{\vee} \in P^{\vee}$ we denote by $s\left(1_{\mu^{\vee}}\right) \in \widehat{\mathbf{U}}_{X, H}$ the expansion in the direction of $P^{\vee,+}$. Here we use the multiplicative structure of $\mathbb{C}\left[P^{\vee}\right]$, i.e., we have

$$
1_{\lambda \vee} 1_{\mu \vee}=1_{\lambda^{\vee}+\mu^{\vee}} .
$$

Finally, we set $u_{\lambda \vee}=\operatorname{Ind} d_{H}^{G}\left(1_{\lambda \vee}\right)$, and we let

$$
\mathbf{U}_{X, G} \subset A F_{X, G}
$$

denote the subspace linearly spanned by the set $\left\{u_{\lambda^{\vee}} ; \lambda^{\vee} \in P^{\vee}\right\}$. For $G=G L_{n}$ we have $\mathbf{U}_{X, G}=\mathbf{U}_{X}^{>}[n]$ with $\mathbf{U}_{X}^{>}$being the spherical Hall algebra of $X$.

Proposition B.1. The following holds.

(a) the map Res $\operatorname{R}_{G}^{H}: \mathbf{U}_{X, G} \rightarrow$ Fun $\left(\right.$ Bun $\left._{H} X, \mathbb{C}\right)$ is injective and takes values in $\widehat{\mathbf{U}}_{X, H}$,

(b) for any $\lambda^{\vee} \in P^{\vee}$ we have

$$
\operatorname{Res}_{G}^{H}\left(u_{\lambda \vee}\right)=\operatorname{Res}_{G}^{H} \circ \operatorname{Ind} d_{H}^{G}\left(1_{\lambda \vee}\right)=\sum_{\sigma \in W} c_{\sigma} 1_{w \lambda \vee}
$$

where

$$
c_{\sigma}=\prod_{\alpha \in \Psi_{\sigma}} q^{1-g} \frac{\zeta_{X}\left(1_{-\alpha^{\vee}}\right)}{\zeta_{X}\left(q 1_{-\alpha^{\vee}}\right)}, \quad \Psi_{\sigma}=\Delta^{+} \cap w^{-1}\left(\Delta^{+}\right)
$$


Statement $(a)$ is proved as in the $G L_{n}$ case, using the nondegeneracy of the natural pairing in $\mathbf{U}_{X, H}$ and the adjunction of $\operatorname{Res}_{G}^{H}$ and $\operatorname{Ind}_{H}^{G}$. Statement $(b)$ is the Gindikin-Karpelevich formula, see e.g. [L1] and [H]. As in the $G L_{n}$ case, it is useful to rephrase the above proposition in combinatorial terms ${ }^{2}$. Put $e_{X}(z)=z^{1-g} \tilde{\zeta}_{X}(z)$, where $\tilde{\zeta}_{X}(z)$ is given by (1.21). Define a symmetrization operator

$$
\Psi_{G}: \mathbb{C}\left[P^{\vee}\right] \rightarrow \mathbb{C}\left[P^{\vee}\right]^{W}, \quad \Psi_{G}\left(1_{\lambda^{\vee}}\right)=\sum_{\sigma \in W} \sigma\left\{\left(\prod_{\alpha \in \Delta^{+}} e_{X}\left(1_{\alpha^{\vee}}\right)\right) \cdot 1_{\lambda^{\vee}}\right\}
$$

We will denote the image of $\Psi_{G}$ by $\mathbf{A}_{G}$. Consider the map

$$
\Xi: \mathbb{C}\left[P^{\vee}\right]^{W} \rightarrow \widehat{\mathbb{C}\left[P^{\vee}\right]}, \quad 1_{\lambda \vee} \mapsto\left(\prod_{\alpha \in \Delta^{+}} e_{X}^{-1}\left(1_{\alpha \vee}\right)\right) 1_{\lambda^{\vee}} .
$$

From Proposition B.1 (b) we see that the following diagram is commutative

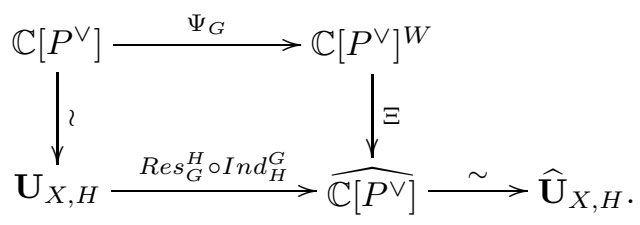

The leftmost vertical map and the rightmost horizontal one are the canonical isomorphisms. We deduce that there exists a (unique) isomorphism $\iota_{G}: \mathbf{U}_{X, G} \rightarrow \mathbf{A}_{G}$ making the next diagram commutative

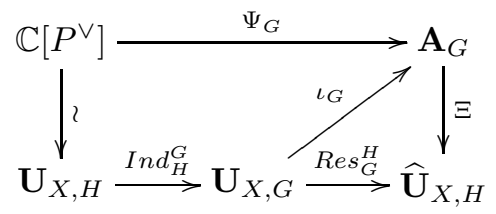

As in the $G L_{n}$ case, we twist the induction product. Let $\rho$ be the half-sum of all positive roots of $G$. We define a new induction product $\dot{I n} d_{H}^{G}: \mathbf{U}_{X, H} \rightarrow \mathbf{U}_{X, G}$ by

$$
\operatorname{In} d_{H}^{G}\left(1_{\lambda \vee}\right)=\operatorname{Ind} d_{H}^{G}\left(1_{2(g-1) \rho \vee} \rho^{\vee}\right) .
$$

Next, define a new symmetrization operator $\dot{\Psi}_{G}: \mathbb{C}\left[P^{\vee}\right] \rightarrow \mathbb{C}\left[P^{\vee}\right]^{W}$ by

$$
\dot{\Psi}_{G}\left(1_{\lambda^{\vee}}\right)=\sum_{\sigma \in W} \sigma\left\{\left(\prod_{\alpha \in \Delta^{+}} \tilde{\zeta}_{X}\left(1_{\alpha} \vee\right) \cdot 1_{\lambda^{\vee}}\right\}\right.
$$

We will denote the image of $\dot{\Psi}_{G}$ by $\dot{\mathbf{A}}_{G}$. It is easy to check that there is a commutative diagram

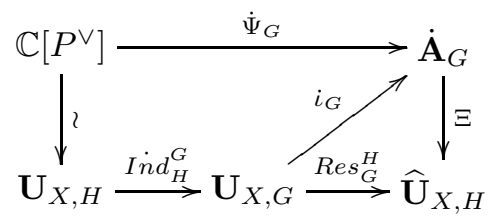

for some (unique) isomorphism $i_{G}: \mathbf{U}_{X, G} \rightarrow \dot{\mathbf{A}}_{G}$. We summarize all this in the following fashion.

\footnotetext{
${ }^{2}$ Note that the sign convention here is different from the one used for $G L_{n}$, see Remark 3.2.2
} 
Proposition B.2. There is a canonical isomorphism $i_{G}: \mathbf{U}_{X, G} \rightarrow \dot{\mathbf{A}}_{G}$ making the following diagram commutative

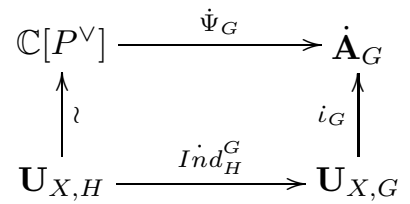

This proposition has a useful corollary. Observe that $\mathbb{C}\left[P^{\vee}\right], \mathbb{C}\left[P^{\vee}\right]^{W}$ do not depend on $X$, while $\dot{\Psi}_{G}$ only depends (polynomially) on the Weyl numbers $\alpha_{1}, \bar{\alpha}_{1}, \ldots, \alpha_{g}, \bar{\alpha}_{g}$. Hence all of these may be defined over the representation ring $R_{a}$ of the torus $T_{a}$. It follows that there exists 'universal' versions $\mathbf{U}_{R_{a}, H}, \mathbf{U}_{R_{a}, G}$ and $\operatorname{In} d_{H}^{G}$ of $\mathbf{U}_{X, H}, \mathbf{U}_{X, G}$ and $\operatorname{In} d_{H}^{G}$ defined over $R_{a}$, depending only on the genus $g$ of $X$, and which specialize to $\mathbf{U}_{X, H}, \mathbf{U}_{X, G}$ and $I \dot{n} d_{H}^{G}$ for any particular choice of $X$. Of course, (B.4) holds for these $R_{a}$-forms, i.e., there is a commutative diagram

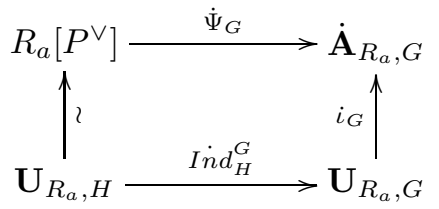

B.3. Let $G^{\vee}$ denote the Langlands dual group to $G$ (a complex reductive group), and let $H^{\vee}$ be a maximal torus of $G^{\vee}$. The lattice of characters $\operatorname{Hom}\left(H^{\vee}, \mathbb{C}^{\times}\right)$of $H^{\vee}$ is identified with $P^{\vee}$. Let $\mathfrak{g}^{\vee}$ and $\mathfrak{h}^{\vee}$ be the respective Lie algebras of $G^{\vee}$ and $H^{\vee}$. Put

$$
\left.C_{\mathfrak{g}^{\vee}}=\left\{(a, b) \in\left(\mathfrak{g}^{\vee}\right)^{g} \times\left(\mathfrak{g}^{\vee}\right)^{g} ; \sum_{r} a d\left(a_{r}\right)\left(b_{r}\right)=0\right\}, \quad C_{\mathfrak{h}^{\vee}}=\mathfrak{h}^{\vee}\right)^{g} \times\left(\mathfrak{h}^{\vee}\right)^{g} .
$$

The torus $T_{s}$ acts on both $C_{\mathfrak{g}^{\vee}}$ and $C_{\mathfrak{h} \vee}$, see Section 2.2. We may apply the method of Section 2.3 verbatim to define a convolution operation

$$
\mu: K^{T_{s} \times H^{\vee}}\left(C_{\mathfrak{h}^{\vee}}\right) \rightarrow K^{T_{s} \times G^{\vee}}\left(C_{\mathfrak{g}^{\vee}}\right) .
$$

We set

$$
\mathbf{C}_{H^{\vee}}^{\prime}=K^{T_{s} \times H^{\vee}}\left(C_{\mathfrak{h} \vee}\right), \quad \mathbf{C}_{G^{\vee}}^{\prime}=K^{T_{s} \times G^{\vee}}\left(C_{\mathfrak{g}^{\vee}}\right) .
$$

Let $R_{G^{\vee}}, R_{H^{\vee}}$ denote the complexified representation rings of $T_{s} \times G^{\vee}, T_{s} \times H^{\vee}$. We have

$$
R_{G^{\vee}}=R_{s}\left[P^{\vee}\right]^{W}, \quad R_{H^{\vee}}=R_{s}\left[P^{\vee}\right]
$$

where $P^{\vee}=\operatorname{Hom}\left(H^{\vee}, \mathbb{C}^{\times}\right)$is the character group of $H^{\vee}$ and $W$ is the Weyl group of $\left(G^{\vee}, H^{\vee}\right)$. The restriction to $\{0\}$ gives rise, as in Section 2.5, to maps

$$
\gamma_{G^{\vee}}: \mathbf{C}_{G^{\vee}}^{\prime} \rightarrow R_{G^{\vee}}, \quad \gamma_{H^{\vee}}: \mathbf{C}_{H^{\vee}}^{\prime} \rightarrow R_{H^{\vee}} .
$$

The map $\gamma_{H^{\vee}}$ is an isomorphism. We conjecture that $\gamma_{G^{\vee}}$ is injective, i.e., that $\mathbf{C}_{G^{\vee}}^{\prime}$ is a torsion free $R_{G^{\vee}}$-module. We denote by $\overline{\mathbf{C}}_{G^{\vee}}$ the quotient of $\mu\left(\mathbf{C}_{H^{\vee}}^{\prime}\right)$ by its torsion submodule. The proof of the following result is identical to that of Proposition 2.8.

Proposition B.3. There is a commutative diagram

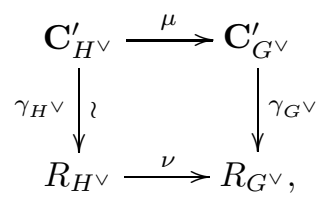


where the map $\nu$ is given by

$$
\nu\left(e^{\lambda^{\vee}}\right)=\sum_{w \in W} w\left(k_{\Delta} \vee e^{\lambda^{\vee}}\right)
$$

where

$$
\begin{gathered}
k_{\Delta \vee}=\prod_{\alpha \in \Delta^{\vee+}} k\left(e^{\alpha}\right), \\
k(z)=(1-z)^{-1}\left(1-p^{-1} z^{-1}\right) \prod_{r}\left(1-x_{r}^{-1} z\right)\left(1-y_{r}^{-1} z\right),
\end{gathered}
$$

and where $\Delta^{\vee+}$ is the set of positive roots of $\mathfrak{g}^{\vee}$.

Set $\mathbf{B}_{G^{\vee}}=\nu\left(R_{H^{\vee}}\right)$. By Proposition B.3, there is a unique isomorphism $j_{G^{\vee}}: \overline{\mathbf{C}}_{G^{\vee}} \rightarrow \mathbf{B}_{G^{\vee}}$ fitting in a commutative diagram

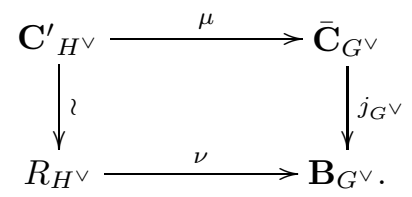

B.4. Identify $T_{a}$ with $T_{s}$ and $R_{a}$ with $R_{s}$ as in Section 3.1. Using (B.6) we get an identification between the maps $\dot{\Psi}_{G}: R\left[P^{\vee}\right] \rightarrow \dot{\mathbf{A}}_{R, G}$ and $\nu: R_{H^{\vee}} \rightarrow \mathbf{B}_{G^{\vee}}$. Combining (B.5) and (B.9), we immediately get

Theorem B.4. There is a canonical isomorphism $\Theta_{R, G}: \overline{\mathbf{C}}_{G} \rightarrow \mathbf{U}_{R, G}$ making the following diagram commutative

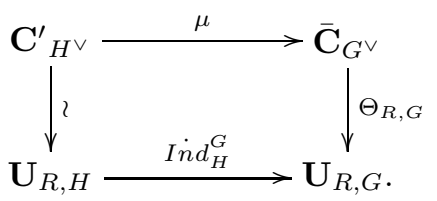

B.5. Fix a point $x \in X\left(\mathbb{F}_{q}\right)$. There is an action $h_{x}$ of the representation ring $R e p_{G} \vee$ on $A F_{X, G}$ by means of the Hecke operators at $x$, see e.g., [F2, Section 2.3]. There is a similar action of $R e p_{H} \vee$ on $A F_{X, H}$, and we have

$$
\begin{array}{r}
L \cdot \operatorname{Ind}_{H}^{G}(f)=\operatorname{Ind}\left(\operatorname{Res}_{G^{\vee}}^{H^{\vee}} L \cdot f\right), \quad L \in \operatorname{Rep}_{G^{\vee}}, \quad f \in A F_{X, H}, \\
\operatorname{Res}_{H^{\vee}}^{G^{\vee}}(L \cdot g)=\operatorname{Res}_{G^{\vee}}^{H^{\vee}}(L) \cdot \operatorname{Res}_{G^{\vee}}^{H^{\vee}}(g), \quad L \in \operatorname{Rep}_{G^{\vee}}, \quad g \in A F_{X, G} .
\end{array}
$$

For $\lambda^{\vee} \in P^{\vee}$ let $e^{\lambda^{\vee}} \in R e p_{H^{\vee}}$ the class of the corresponding one-dimensional module. A direct computation shows that

$$
e^{\lambda^{\vee}} \cdot 1_{\mu^{\vee}}=1_{\lambda^{\vee}+\mu^{\vee}}=1_{\lambda^{\vee}} 1_{\mu^{\vee}}, \quad \lambda^{\vee}, \mu^{\vee} \in P^{\vee} .
$$

The product on the left hand side is the Hecke action while the product on the right hand side is the multiplication in the group ring $\mathbb{C}\left[P^{\vee}\right]$. Note that the Hecke action $h_{x}$ on $\mathbf{U}_{X, H}$ and hence on $\mathbf{U}_{X, G}$ is independent of the choice of the point $x$. On the other hand, there is a natural action $\rho$ of the representation ring $R_{G \vee}$ on $\overline{\mathbf{C}}_{G^{\vee}}$ by tensor product.

Theorem B.5. The isomorphism $\Theta_{R, G}$ intertwines the above actions of Rep $G^{\vee}$ on $\overline{\mathbf{C}}_{G^{\vee}}$ and $\mathbf{U}_{R, G}$ respectively, i.e., we have $\Theta_{R, G} \circ \rho(L)=h_{x}(L) \circ \Theta_{R, G}$ for any $L \in R e p_{G^{\vee}}$. 
O. SCHIFFMANN, E. VASSEROT

Proof. The restriction map $\gamma_{G^{\vee}}: \mathbf{C}_{G^{\vee}}^{\prime} \rightarrow R_{G^{\vee}}$ is $R_{G^{\vee}}$-linear. Likewise, the map $\gamma_{H^{\vee}}: \mathbf{C}_{H^{\vee}}^{\prime} \rightarrow$ $R_{H^{\vee}}$ is $R_{H^{\vee}}$-linear. Letting $R_{G^{\vee}}$ act on $\mathbf{C}_{H^{\vee}}^{\prime}$ and $R_{H^{\vee}}$ by means of the embedding $R_{G^{\vee}} \subset R_{H^{\vee}}$, we see from (B.7) that $\mu, \nu$ are $R_{G^{\vee}}$-linear as well, and hence that $j_{G^{\vee}}$ is $R_{G^{\vee}}$-linear. By (B.12) the identification $\mathbf{U}_{R, H} \simeq R\left[P^{\vee}\right]$ is $R_{H^{\vee}}$-linear. Let $R_{G^{\vee}}$ act on $R\left[P^{\vee}\right]$ and $\mathbf{U}_{R, H}$ by means of the embedding $R_{G^{\vee}} \subset R_{G^{\vee}}$. By construction, the map $\dot{\Psi}_{G}$ is $R_{G^{\vee}}$-linear. By (B.11), the map $\operatorname{In} d_{H}^{G}$ is also $R_{G^{\vee}}$-linear. But then $i_{G}$ is $R_{G^{\vee}}$-linear as well. We have shown that $j_{G^{\vee}}$ and $i_{G}$ are $R_{G^{\vee}}$-linear. The identification $\dot{\mathbf{A}}_{G} \simeq \mathbf{B}_{G^{\vee}}$ is obviously $R_{G^{\vee}}$-linear. The theorem follows.

B.6. To finish we describe, as in Section 3.3, the image under the isomorphism $\Theta_{K, G}$ of the skyscraper sheaf $\left[\mathcal{O}_{\{0\}}\right] \in \mathbf{C}_{G^{\vee}}^{\prime} \otimes_{R} K$. The proof is the same as in the case of $\mathfrak{g}=\mathfrak{g l}_{r}$, see Proposition 3.6. Let us denote by $\mathcal{B}=G / B$ the flag variety of $G$.

Proposition B.6. We have

$$
\Theta_{K, G}\left(\left[\mathcal{O}_{\{0\}}\right]\right)=(-1)^{\operatorname{dim} \mathcal{B}} \frac{q^{-g \operatorname{dim} \mathcal{B}}}{\# \mathcal{B}\left(\mathbb{F}_{q}\right)} \operatorname{Ind}_{H}^{G}\left(1_{-2 \rho^{\vee}} \prod_{\sigma \in \Delta^{+}} \prod_{l=1}^{g}\left(1-\alpha_{l} 1_{\sigma^{\vee}}\right)\left(1-\bar{\alpha}_{l} 1_{\sigma^{\vee}}\right)\right)
$$

where $\Delta^{+}$is the set of positive roots of $(G, B)$.

\section{REFERENCES}

[BS] I. Burban, O. Schiffmann, On the Hall algebra of an elliptic curve, I., preprint math.AG/0505148, (2005).

[CG] N. Chriss, V. Ginzburg, Representation theory and complex geometry, Birkhaüser (1996).

[D] P. Deligne, Constantes des équations fonctionelles des fonctions L, in: Lecture Notes in Math., 349, p. 501-597, Springer-Verlag (1973).

[FJMM] B. Feigin, M. Jimbo, T. Miwa, E. Mukhin, Symmetric polynomials vanishing on the shifted diagonals and Macdonald polynomials, Int. Math. Res. Not. 2003, no. 18, 1015-1034.

[FO] B. Feigin, A. Odesskii, Vector bundles on an elliptic curve and Sklyanin algebras, Topics in quantum groups and finite-type invariants, 65-84, Amer. Math. Soc. Transl. Ser. 2, 185, (1998).

[FT] B. Feigin, A. Tsymbaliuk, Heisenberg action in the equivariant K-theory of Hilbert schemes via Shuffle Algebra, preprint arXiv:0904.1679 (2009).

[F1] D. Fratila, On the whole Hall algebra of an elliptic curve, in preparation.

[F2] E. Frenkel, Lectures on the Langlands program and conformal field theory, Frontiers in number theory, physics, and geometry. II, 387-533, Springer, Berlin, (2007).

[FGV] E. Frenkel, D. Gaitsgory, K. Vilonen, On the geometric Langlands conjecture, J. Amer. Math. Soc. 15 (2002), no. 2, 367-417.

[Ga] D. Gaitsgory, Automorphic sheaves and Eisenstein series, Thesis, Tel-Aviv University (1997).

[Gr] I. Grojnowski, Instantons and affine algebras I: the Hilbert scheme and vertex operators, Math. Res. Letters 3 (1996), 275-291.

[H] G. Harder, Chevalley Groups over Function Fields and Automorphic Forms, Ann. Math., Vol. 100, No. 2, pp. 249-306

[HN] G. Harder, M.S. Narasimhan, On the cohomology groups of moduli spaces of vector bundles on curves, Math. Ann. 212 (1974/75), 215-248.

[K] M. Kapranov, Eisenstein series and quantum affine algebras, Algebraic geometry, 7. J. Math. Sci. (New York) 84 (1997), no. 5, 1311-1360.

[L1] R. P. Langlands, Euler products, A James K. Whittemore Lecture in Mathematics given at Yale University, 1967. Yale Mathematical Monographs, 1. Yale University Press, New Haven, Conn.-London, 1971.

[L2] A. Lascoux, Symmetric functions and combinatorial operators on polynomials, CBMS Regional Conference Series in Mathematics, 99, (2003).

[L3] G. Laumon, Faisceaux automorphes asociées aux séries d'Eisenstein, Automorphic forms, Shimura varieties, and $L$-functions, Vol. I (Ann Arbor, MI, 1988), 227-281.

[L4] G. Laumon, La transformation de Fourier géométrique et ses applications, Proceedings of the International Congress of Mathematicians, Vol. I, II (Kyoto, 1990), 437-445, Math. Soc. Japan, Tokyo, 1991.

[L5] G. Lusztig, Introduction to quantum groups, Birkhaüser, (1992).

[M1] I.G. Macdonald, Symmetric functions and Hall polynomials, second edition, Oxford Math. Mon., (1995).

[M2] J.S. Milne, Abelian varieties, in Arithmetic geometry (Storrs, Conn., 1984), 103-150, Springer, New York, (1986).

[R1] C. Ringel, Hall algebras and quantum groups, Invent. Math. 101 (1990), no. 3, 583-591. 
[R2] M. Rosso, Marc, Quantum groups and quantum shuffles, Invent. Math. 133 (1998), no. 2, 399-416.

[SGA6] P. Berthelot, A. Grothendieck, A. Illusie, Théorie des intersections et Théorème de Riemann-Roch, Lect. Notes Math. 162 (1971).

[S1] O. Schiffmann, Lectures on Hall algebras I, II, preprint arXiv:0611617, to appear in Comptes-Rendus de l'école doctorale de Grenoble, (2006).

[S2] O. Schiffmann, Spherical Hall algebras of curves and Harder-Narasimhan stratas, preprint arXiv:1002.0987 (2010).

[S3] O. Schiffmann, On the Hall algebra of an elliptic curve, II, preprint arXiv:math/0508553 (2005).

[SV1] O. Schiffmann, E. Vasserot, The elliptic Hall algebra, Cherednick Hecke algebras and Macdonald polynomials, preprint arXiv:0802.4001, to appear in Compositio, (2008).

[SV2] O. Schiffmann, E. Vasserot, The elliptic Hall algebra and the K-theory of the Hilbert scheme of $\mathbb{A}^{2}$, preprint arXiv:0905.2558 (2009).

[T] R.W. Thomason, Les K-groupes d'un schéma éclaté et une formule d'intersection excédentaire, Invent. Math. 112 (1993), 195-215.

[V] E. Vasserot, Sur l'anneau de cohomologie du schéma de Hilbert de $\mathbf{C}^{2}$,. C. R. Acad. Sci. Paris Sér. I Math. 332 (2001), no. 1, 7-12.

[VV] M. Varagnolo, E. Vasserot, On the K-theory of the cyclic quiver variety. Internat. Math. Res. Notices (1999), no. 18, 1005-1028.

O. Schiffmann, olive@math.jussieu.fr,

Département de Mathématiques, Université de Paris 6, 175 rue du Chevaleret, 75013 Paris, FRANCE.

E. Vasserot, vasserot@math.jussieu.fr,

Département de Mathématiques, Université de Paris 7, 175 rue du Chevaleret, 75013 Paris, FRANCE. 